Métodos de programação quadrática convexa esparsa e suas aplicações em projeções em poliedros

Jeinny Maria Peralta Polo 



\title{
Métodos de programação quadrática convexa esparsa e suas aplicações em projeções em poliedros
}

\author{
Jeinny Maria Peralta Polo
}

Orientadora: Profa. Dra. Marina Andretta

\begin{abstract}
Dissertação apresentada ao Instituto de Ciências Matemáticas e de Computação - ICMC-USP, como parte dos requisitos para obtenção do título de Mestre em Ciências - Ciências de Computação e Matemática Computacional. VERSÃO REVISADA
\end{abstract}


Ficha catalográfica elaborada pela Biblioteca Prof. Achille Bassi e Seção Técnica de Informática, ICMC/USP, com os dados fornecidos pelo(a) autor(a)

\begin{tabular}{|c|c|}
\hline \multirow[t]{3}{*}{ P 778m } & $\begin{array}{l}\text { Peralta Polo, Jeinny Maria } \\
\quad \text { Métodos de programação quadrática convexa esparsa } \\
\text { e suas aplicações em projeções em poliedros / Jeinny } \\
\text { Maria Peralta Polo; orientadora Marina Andretta. -- } \\
\text { São Carlos, } 2013 \text {. } \\
\quad 108 \text { p. }\end{array}$ \\
\hline & $\begin{array}{l}\text { Dissertação (Mestrado - Programa de Pós-Graduação } \\
\text { em Ciências de Computação e Matemática } \\
\text { Computacional) -- Instituto de Ciências Matemáticas } \\
\text { e de Computação, Universidade de São Paulo, } 2013 .\end{array}$ \\
\hline & $\begin{array}{l}\text { 1. Minimização com restrições lineares. 2. Projeção } \\
\text { esparsa. 3. Programação quadrática convexa esparsa. } \\
\text { I. Andretta, Marina, orient. II. Título. }\end{array}$ \\
\hline
\end{tabular}


Aos meus amados pais e meu esposo. 



\section{Agradecimentos}

À professora Marina pela excelente orientação oferecida na realização deste trabalho e pela motivação, colaboração e apoio nos momentos difíceis.

Aos professores que tive, porque deles aprendi muitas coisas.

A toda minha família, especialmente meus pais, Belsy e Alvaro, pelo apoio e amor que sempre me dão.

Aos meus amigos e colegas pela ajuda, especialmente minha querida amiga Yaneth, que apesar da distância sempre está comigo.

À FAPESP, pelo apoio financeiro.

Finalmente, agradeço ao meu principal apoio e incentivo ao longo destes anos, meu amado esposo Alfredo, pelo seu grande amor, compreensão e dedicação. 



\section{Resumo}

O problema de minimização com restrições lineares é importante, não apenas pelo problema em si, que surge em várias áreas, mas também por ser utilizado como subproblema para resolver problemas mais gerais de programação não-linear. GENLIN é um método eficiente para minimização com restrições lineares para problemas de pequeno e médio porte. Para que seja possível a implementação de um método similar para grande porte, é necessário ter um método eficiente, também para grande porte, para projeção de pontos no conjunto de restrições lineares.

O problema de projeção em um conjunto de restrições lineares pode ser escrito como um problema de programação quadrática convexa. Neste trabalho, estudamos e implementamos métodos esparsos para resolução de problemas de programação quadrática convexa apenas com restrições de caixa, em particular o clássico método Moré-Toraldo e o "método" NQC. O método Moré-Toraldo usa o método dos Gradientes Conjugados para explorar a face da região factível definida pela iteração atual, e o método do Gradiente Projetado para mudar de face. O "método" NQC usa o método do Gradiente Espectral Projetado para definir em que face trabalhar, e o método de Newton para calcular o minimizador da quadrática reduzida a esta face.

Utilizamos os métodos esparsos Moré-Toraldo e NQC para resolver o problema de projeção de GENLIN e comparamos seus desempenhos. 



\section{Abstract}

The linearly constrained minimization problem is important, not only for the problem itself, that arises in several areas, but because it is used as a subproblem in order to solve more general nonlinear programming problems. GENLIN is an efficient method for solving small and medium scaled linearly constrained minimization problems. To implement a similar method to solve large scale problems, it is necessary to have an efficient method to solve sparse projection problems onto linear constraints.

The problem of projecting a point onto a set of linear constraints can be written as a convex quadratic programming problem. In this work, we study and implement sparse methods to solve box constrained convex quadratic programming problems, in particular the classical Moré-Toraldo method and the NQC "method". The Moré-Toraldo method uses the Conjugate Gradient method to explore the face of the feasible region defined by the current iterate, and the Projected Gradient method to move to a different face. The NQC "method" uses the Spectral Projected Gradient method to define the face in which it is going to work, and the Newton method to calculate the minimizer of the quadratic function reduced to this face.

We used the sparse methods Moré-Toraldo and NQC to solve the projection problem of GENLIN and we compared their performances. 



\section{Sumário}

$\begin{array}{lc}\text { Introdução } & 3\end{array}$

1 Programação quadrática convexa com restrições de caixa $\quad 9$

1.1 Método Moré-Toraldo . . . . . . . . . . . . . . . . . . . . . 13

1.1 .1 Busca projetada . . . . . . . . . . . . 18

1.1.2 Resultados de convergência . . . . . . . . . . . . . 22

1.1.3 Resultados numéricos . . . . . . . . . . . . . . . . 25

1.2 "Método" NQC . . . . . . . . . . . . . . . . . . . 29

1.3 Resultados numéricos . . . . . . . . . . . . . . . . 30

2 Método para resolução de problemas de minimização com restrições lineares $\quad 45$

2.1 Algoritmos "irrestritos" . . . . . . . . . . . . . . . . . . 47

2.1.1 Algoritmo "irrestrito" com regiões de confiança . . . . . . 49

2.1.2 Algoritmo "irrestrito" com busca linear . . . . . . . . . . 51

2.1.3 Cálculo da base do núcleo . . . . . . . . . . . . . . 52

2.2 Método do Gradiente Espectral Projetado Parcial . . . . . . . . 54

2.3 Método de restrições ativas para problemas com restrições lineares 57

2.4 Projeção . . . . . . . . . . . . . . . . . . . . . . . . . . 58

2.5 Teoria de convergência . . . . . . . . . . . . . . . 59

2.6 Detalhes de implementação . . . . . . . . . . . . . . . . . 66

2.6.1 Detalhes da implementação de GENLIN-GPCG . . . . . 67

2.6.2 Detalhes da implementação de GENLIN-NQC . . . . . . 69

2.7 Resultados numéricos . . . . . . . . . . . . . . . . . 70

3 Conclusões e trabalhos futuros $\quad 95$ 
A Provas de teoremas, proposições e propriedades

Referências 


\section{Introdução}

O problema de minimização com restrições lineares é importante, não apenas pelo problema em si, que surge em várias áreas, mas também por ser utilizado como subproblema para resolver problemas mais gerais de programação não-linear.

Estamos interessados em resolver o seguinte problema:

$$
\begin{array}{ll}
\text { Minimizar } & f(x) \\
\text { sujeita a } & x \in \Omega=\left\{x \in \mathbb{R}^{n} \mid A x=b, C x \leq d, l \leq x \leq u\right\}
\end{array}
$$

onde $f$ é uma função em $\mathcal{C}^{2}, x \in \mathbb{R}^{n}, A \in \mathbb{R}^{r \times n}, b \in \mathbb{R}^{r}, C \in \mathbb{R}^{m \times n}, d \in \mathbb{R}^{m}, l \in$ $(\mathbb{R} \cup\{-\infty\})^{n}, u \in(\mathbb{R} \cup\{+\infty\})^{n}$ e $[l]_{i}<[u]_{i}, 1 \leq i \leq n$. Daqui em diante, []$_{i}$ denota a $i$-ésima componente de um vetor.

Um método bastante usado para resolver (1) é o método de restrições ativas. GENLIN, apresentado em Andretta et al. (2010), é a implementação de um método de restrições ativas para problemas de pequeno e médio porte, que faz parte do Projeto TANGO (Trustable Algorithms for Nonlinear General Optimization) ${ }^{1}$. Neste método, o conjunto viável é dividido em faces. Cada face é determinada por um conjunto de restrições lineares de igualdade. A cada iteração, decide-se se deve ser encontrado o minimizador da função restrito à face ou se a face dever ser abandonada. No primeiro caso, resolve-se um subproblema irrestrito utilizando busca linear com Newton truncado ou regiões de confiança com Moré-Sorensen (Moré e Sorensen (1983)), tomando-se o cuidado de não violar nenhuma das restrições de desigualdade que não foram

\footnotetext{
${ }^{1}$ Disponível em www.ime.usp.br/ egbirgin/tango .
} 
consideradas na resolução do subproblema. No segundo caso, usa-se o método do Gradiente Espectral Projetado Parcial (PSPG) (inspirado no método do Gradiente Espectral Projetado, apresentado em Birgin et al. (2000, 2001)), que necessita fazer projeções em um subconjunto de restrições do problema original (1).

Para que seja possível a implementação de um método similar para grande porte, é necessário ter um método eficiente, também para grande porte, para projeção de pontos no conjunto de restrições lineares (usado em PSPG).

O problema de projeção em um conjunto de restrições lineares pode ser escrito como um problema de programação quadrática convexa,

$$
\begin{array}{cl}
\text { Minimizar } \quad & \frac{1}{2}\|x-\tilde{x}\|_{2}^{2} \equiv \frac{1}{2} x^{T} I x-\tilde{x}^{T} x \\
\text { sujeita a } \quad & A x=b, \\
& \tilde{C} x \leq \tilde{d} \\
& l \leq x \leq u
\end{array}
$$

onde $\tilde{x} \in \mathbb{R}^{n}$ é o ponto que queremos projetar, $I$ é a matriz identidade em $\mathbb{R}^{n \times n}, \tilde{C} \in \mathbb{R}^{\tilde{m} \times n}$ e $\tilde{d} \in \mathbb{R}^{\tilde{m}}$ correspondem às restrições lineares de desigualdade utilizadas na projeção.

Há muitos anos problemas de programação quadrática convexa vem sendo estudados e existem alguns métodos para sua resolução. Em Goldfarb e Idnani (1983) foi apresentado um método dual de restrições ativas, eficiente e numericamente estável, para resolver problemas densos de programação quadrática definidos positivos. Este método, que usa como ponto inicial o minimizador do problema irrestrito, foi estendido para problemas mal condicionados por Powell (1985) e reformulado por Boland (1997) para problemas de programação quadrática semi-definidos positivos. Uma outra reformulação do método Goldfarb-Idnani foi proposta por Bartlett e Biegler (2006), em que o método do complemento de Schur Gill et al. (1990) é usado para resolver sistemas lineares.

Estamos interessados em estudar a aplicação de métodos de programação quadrática convexa esparsa para resolver o problema (2). Uma outra maneira de calcular a projeção é considerar o problema dual de (2), dado por 


$$
\begin{array}{ll}
\text { Minimizar } & \frac{1}{2} y^{T} \tilde{A} \tilde{A}^{T} y+(\tilde{b}-\tilde{A} \tilde{x})^{T} y \\
\text { sujeita a } & y_{i} \geq 0, \quad i=r+1, r+2, \ldots, r+\tilde{m}+2 n
\end{array}
$$

onde $\tilde{A} \in \mathbb{R}^{(r+\tilde{m}+2 n) \times n}, \tilde{A}=\left[\begin{array}{r}A \\ \tilde{C} \\ -I \\ I\end{array}\right]$ e $\tilde{b} \in \mathbb{R}^{r+\tilde{m}+2 n}, \tilde{b}=\left[\begin{array}{r}b \\ \tilde{d} \\ -\ell \\ u\end{array}\right]$.

Note que (3) é um problema de programação quadrática com restrições de caixa. Este problema tem sido muito estudado.

Em Coleman e Hulbert. (1989) mostrou-se que é possível implementar eficientemente um método direto de restrições ativas para resolver problemas de programação quadráticas com restrições de caixa de grande porte.

Em Friedlander e Martínez (1994) introduz-se um novo método para problemas de grande porte, que combina Gradiente Conjugado com Gradiente Projetado como em Moré e Toraldo (1991) e outros métodos conhecidos. Neste método, uma nova estratégia para a decisão de sair ou ficar na face é introduzida, que torna possível obter convergência finita mesmo para uma Hessiana singular e uma solução dual degenerada. Um ano depois, em Friedlander et al. (1995), foi apresentada uma modificação para este novo método, em que o método Barzilai-Borwein (introduzido por Barzilai e Borwein (1988) e analisado por Raydan (1993)) é usado em vez do clássico método do Gradiente Conjugado para minimização dentro das faces.

Em Bielschowski et al. (1997) apresentou-se um algoritmo de restrições ativas, que pode usar diferentes técnicas de minimização irrestrita em cada face, dependendo da dimensão desta. Dois problemas de projeção em poliedro foram mostrados nos testes apresentados nesse artigo.

Em Dostál (2003) foi apresentada uma modificação do "proportioning algorithm", desenvolvido em Dostál (1997). O "proportioning algorithm" é um algoritmo do tipo restrições ativas para problemas de grande porte que, para explorar uma face, usa o método do Gradiente Conjugado até que seja gerada uma iteração desproporcional (conceito introduzido nesse artigo). 
Em Dostál e Schöberl (2005) apresentou-se uma nova modificação do "proportioning algorithm", onde é mostrada a taxa de convergência e a propriedade de terminação finita, mesmo para problemas cuja solução não satisfaz a condição de complementaridade estrita.

Em Dai e Fletcher (2005) estudaram-se os dois métodos Barzilai-Borwein projetado (propostos por Birgin et al. (2000), para minimização de funções diferenciáveis em conjuntos convexos fechados), para problemas de programação quadrática de grande porte com restrições de caixa, fazendo uma adaptação à busca linear não monótona. Os métodos Barzilai-Borwein projetado em Birgin et al. (2000) são a combinação do clássico método do gradiente projetado com a estratégia de busca linear não monótona (sugerida por Grippo et al. (1986), para métodos de Newton) e os tamanhos do passo espectrais (introduzidos em Barzilai e Borwein (1988) e analisados por Raydan (1993)).

Em Zhou et al. (2006), inspirado em Dai e Fletcher (2005) e Birgin et al. (2000), são propostos e analisados métodos de gradiente projetado do tipo monótono para problemas de programação quadrática com restrições de caixa de grande porte. Nestes métodos, no cálculo do tamanho do passo, são usados gradientes projetados contínuos ou descontínuos em vez de gradientes negativos.

Neste trabalho, estudamos e implementamos o método Moré-Toraldo, apresentado em Moré e Toraldo (1991), por ser um método clássico para resolução de problemas de programação quadrática convexa com restrições de caixa de grande porte. Neste método, como em GENLIN, a região factível é dividida em faces. Cada face é determinada pelo conjunto de restrições ativas. Uma variável $[x]_{i}$ é ativa se $[x]_{i}=[l]_{i}$ ou $[x]_{i}=[u]_{i}$. A cada iteração, o método Moré-Toraldo usa o método de Gradientes Conjugados para explorar a face da região factível, e o método de Gradiente Projetado para mudar de face. Além do método Moré-Toraldo, estudamos o "método" NQC, desenvolvido e implementado pela Profa. Dra. Marina Andretta, durante seu pós-doutorado, em parceria com os professores José Mario Martínez e Mikhail Solodov, para resolução de problemas de programação quadrática convexa com restrições de caixa. O "método" NQC, sigla para Newton para Quadráticas convexas em Caixas, usa o método do Gradiente Espectral Projetado para definir em que 
face trabalhar, e o método de Newton para calcular o minimizador da quadrática reduzida a esta face.

Utilizamos o método Moré-Toraldo e o "método" NQC em GENLIN para resolver o problema de projeção e comparamos seus desempenhos.

Neste trabalho, os capítulos estão organizados da maneira que segue. No Capítulo 1, descrevemos dois métodos para resolução de problemas de programação quadrática com restrições de caixa, o Método Moré-Toraldo e o "método" NQC, assim como algumas definições, proposições e teoremas utéis para uma melhor compreensão dos problemas de programação quadrática e dos métodos para a resolução destes problemas. Apresentamos detalhes da implementação de cada um dos métodos e resultados numéricos comparando estes métodos entre si. No Capítulo 2, descrevemos em detalhes GENLIN, um método eficiente para resolução do problema de minimização com restrições lineares. Apresentamos resultados numéricos comparando o desempenho da versão original de GENLIN com as duas novas versões: uma que utiliza o método Moré-Toraldo e outra que utiliza o "método" NQC para calcular projeções esparsas. Por fim, no Capítulo 3, apresentamos as conclusões finais deste trabalho e sugestões para trabalhos futuros. 


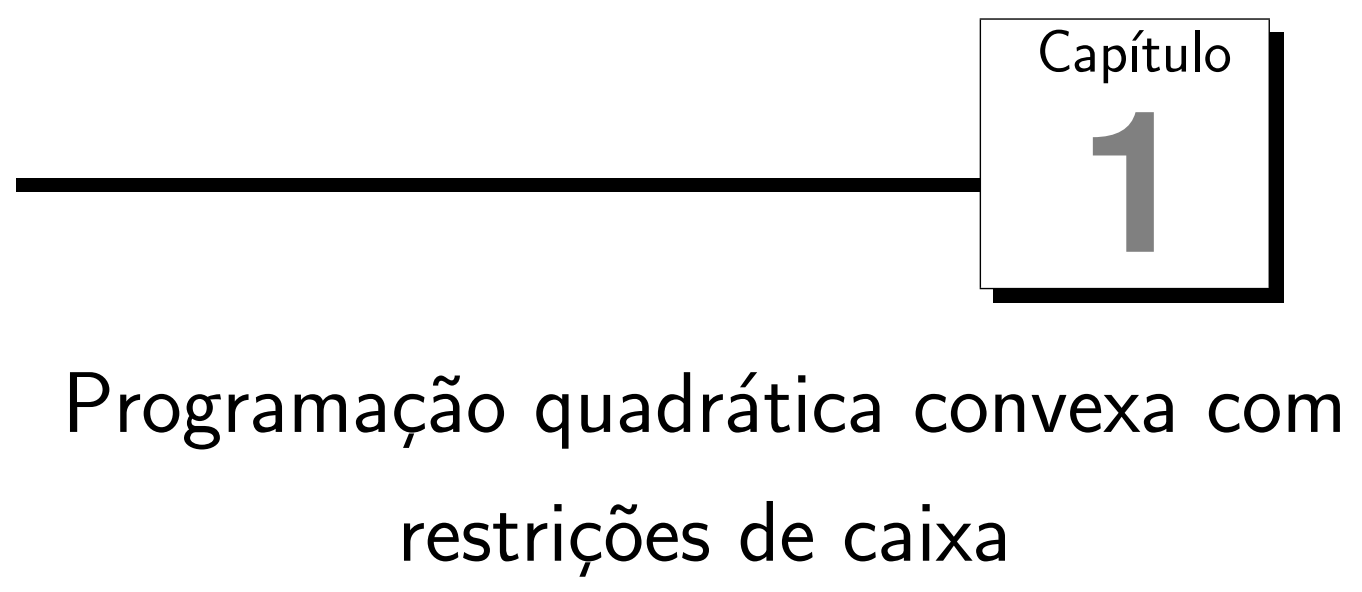

Neste capítulo, iremos apresentar dois métodos para resolução de problemas de programação quadrática convexa com restrições de caixa, o Método Moré-Toraldo e o "método" NQC. Primeiramente, apresentaremos algumas definições, proposições e teoremas utéis para uma melhor compreensão do problema e dos métodos.

O problema de programação quadrática com restrições de caixa é definido como segue:

$$
\begin{array}{ll}
\text { Minimizar } & q(x) \\
\text { sujeita a } & x \in \Omega_{\square},
\end{array}
$$

onde $q: \mathbb{R}^{n} \rightarrow \mathbb{R}$ é uma função quadrática e

$$
\Omega_{\square}=\left\{x \in \mathbb{R}^{n}: l \leq x \leq u\right\},
$$

é a região factível, sendo $l \in(\mathbb{R} \cup\{-\infty\})^{n}, u \in(\mathbb{R} \cup\{+\infty\})^{n}$ e $[l]_{i}<[u]_{i}$.

Observação 1.1. Note que a região factível $\Omega_{\square}$ é um conjunto convexo.

No Teorema 1.1, que é provado no Apêndice A, afirmamos que a solução $x^{*}$ do problema (1.1) é única se a função $q$ é estritamente convexa sobre o 
conjunto $\Omega_{\square}$.

Teorema 1.1. O problema de programação quadrática com restrições de caixa (1.1) tem solução única $x^{*}$ quando q é estritamente convexa na região factivel (1.2).

O Teorema 1.2 mostra uma condição necessária e suficiente para que um ponto seja solução do problema (1.1) (veja Bertsekas (1999), proposição 2.1.2).

Teorema 1.2. Considere $q$ uma função convexa sobre o conjunto $\Omega_{\square} \cdot O$ ponto $x^{*}$ é minimizador da função q sobre o conjunto $\Omega_{\square}$ se, e somente se, $\nabla q\left(x^{*}\right)^{T}\left(x-x^{*}\right) \geq 0$, para todo $x \in \Omega_{\square}$.

Para a prova, veja Apêndice A.

Assim, pelo Teorema 1.2, a solução $x^{*}$ do problema (1.1) pode ser caracterizada como a solução da desigualdade variacional

$$
\nabla q\left(x^{*}\right)^{T}\left(x-x^{*}\right) \geq 0, \quad \forall x \in \Omega_{\square} .
$$

Se o conjunto $\Omega_{\square}$, definido por (1.2), é a região factível, qualquer solução da desigualdade variacional (1.3) satisfaz as condições Karush-Kuhn-Tucker ou condições necessárias de primeira ordem KKT para o problema (1.1) (veja, por exemplo, Nocedal e Wright (1999)). As condições são ditas de primeira ordem pois utilizam apenas informação do gradiente da função objetivo e das restrições.

As condições necessárias de primeira ordem KKT para que um ponto $x^{*}$ seja solução de (1.1) são

$$
\begin{aligned}
\nabla q\left(x^{*}\right)-\sum_{i \in \mathcal{I}_{l}}\left[\lambda^{*}\right]_{i} e_{i}+\sum_{i \in \mathcal{I}_{u}}\left[\mu^{*}\right]_{i} e_{i}=0, & \\
{\left[x^{*}-l\right]_{i} \geq 0, } & \forall i \in \mathcal{I}_{l}, \\
{\left[u-x^{*}\right]_{i} \geq 0, } & \forall i \in \mathcal{I}_{u}, \\
{\left[\lambda^{*}\right]_{i} } & \geq 0, \quad \forall i \in \mathcal{I}_{l}, \\
{\left[\mu^{*}\right]_{i} } & \geq 0, \quad \forall i \in \mathcal{I}_{u}, \\
{\left[\lambda^{*}\right]_{i}\left[x^{*}-l\right]_{i} } & =0, \quad \forall i \in \mathcal{I}_{l}, \\
{\left[\mu^{*}\right]_{i}\left[u-x^{*}\right]_{i} } & =0, \quad \forall i \in \mathcal{I}_{u},
\end{aligned}
$$


com $\left[\lambda^{*}\right]_{i},\left[\mu^{*}\right]_{i} \in \mathbb{R}, e_{i} i$-ésima coluna da matriz identidade, $\mathcal{I}_{l}$ e $\mathcal{I}_{u}$ conjunto dos índices das restrições $[l]_{i} \leq[x]_{i}$, para $[l]_{i} \in \mathbb{R}$ e $[x]_{i} \leq[u]_{i}$, para $[u]_{i} \in \mathbb{R}$, respectivamente. Note que $\mathcal{I}_{l}$ e $\mathcal{I}_{u}$ não necessariamente são o conjunto $\{i: 1 \leq i \leq n\}$, pois é possível que $[l]_{i}=-\infty$ ou $[u]_{i}=\infty$ para algum $1 \leq i \leq n$.

Estas condições podem ser escritas como: se um ponto $x^{*} \in \Omega_{\square}$ é solução de (1.1), então

$$
\begin{aligned}
& {\left[\nabla q\left(x^{*}\right)\right]_{i}=0, \quad \text { se }\left[x^{*}\right]_{i} \in\left([l]_{i},[u]_{i}\right),} \\
& {\left[\nabla q\left(x^{*}\right)\right]_{i} \geq 0, \quad \text { se }\left[x^{*}\right]_{i}=[l]_{i}} \\
& {\left[\nabla q\left(x^{*}\right)\right]_{i} \leq 0, \quad \text { se }\left[x^{*}\right]_{i}=[u]_{i}}
\end{aligned}
$$

A seguir, apresentamos três definições e duas proposições apropriadas para restrições de caixa, úteis para definir uma solução aproximada do problema (1.1).

Definição 1.1. A projeção ortogonal de um ponto $x$ no conjunto viável $\Omega_{\square}$ é dada da seguinte maneira:

$$
P_{\Omega_{\square}}(x)= \begin{cases}{[x]_{i},} & \text { se }[l]_{i} \leq[x]_{i} \leq[u]_{i} \\ {[l]_{i},} & \text { se }[x]_{i}<[l]_{i} \\ {[u]_{i},} & \text { se }[x]_{i}>[u]_{i}\end{cases}
$$

Definição 1.2. O gradiente projetado contínuo no ponto $x$ é dado por:

$$
P_{\Omega_{\square}}(x-\nabla q(x))-x,
$$

onde $P_{\Omega_{\square}}$ é a projeção (1.6).

Definição 1.3. O gradiente projetado descontínuo no ponto $x$ é dado por:

$$
\left[\nabla_{\Omega_{\square}} q(x)\right]_{i}= \begin{cases}{[\nabla q(x)]_{i},} & \text { se }[x]_{i} \in\left([l]_{i},[u]_{i}\right), \\ \min \left\{[\nabla q(x)]_{i}, 0\right\}, & \text { se }[x]_{i}=[l]_{i}, \\ \max \left\{[\nabla q(x)]_{i}, 0\right\}, & \text { se }[x]_{i}=[u]_{i} .\end{cases}
$$

O gradiente projetado descontínuo é o negativo do gradiente projetado definido em Calamai e Moré (1987) para um conjunto convexo fechado qualquer. 
Note que $\nabla_{\Omega_{\square}} q(x)$ pode ser descontínuo na fronteira de $\Omega_{\square}$.

Proposição 1.1. Um ponto $x^{*}$ viável satisfaz $P_{\Omega_{\square}}\left(x^{*}-\nabla q\left(x^{*}\right)\right)-x^{*}=0$, se, e somente se, este ponto também satisfaz as condições KKT (1.4).

Para a prova, veja o Apêndice A.

Proposição 1.2. Um ponto $x^{*}$ viável satisfaz $\nabla_{\Omega_{\square}} q\left(x^{*}\right)=0$, se, e somente se, este ponto também satisfaz as condições KKT (1.4).

Para a prova, veja o Apêndice A.

Com base na Proposição 1.2, definimos um minimizador aproximado do problema (1.1) como um vetor $x \in \Omega_{\square}$ tal que

$$
\left\|\nabla_{\Omega_{\square}} q(x)\right\|_{2} \leq \tau\left\|\nabla q\left(x_{0}\right)\right\|_{2},
$$

sendo $x_{0} \in \Omega_{\square}$ um ponto inicial dado, e $\tau \in(0,1)$ uma tolerância.

Uma definição alternativa para o minimizador aproximado do problema (1.1) é

$$
\left\|P_{\Omega_{\square}}(x-\nabla q(x))-x\right\|_{2} \leq \tau\left\|\nabla q\left(x_{0}\right)\right\|_{2},
$$

$\operatorname{com} x \in \Omega_{\square}$.

Uma vantagem de (1.7) sobre (1.8) é que, se substituímos $q$ por $\alpha q$, para algum $\alpha>0$, apenas (1.7) é invariante à escala. De fato, se substituímos $q$ por $\alpha q$ em (1.8), e $\Omega_{\square}$ é limitado, então (1.8) é satisfeito para qualquer $x \in \Omega_{\square}$, se $\alpha$ é suficientemente grande.

A seguir, definimos formalmente face e conjunto ativo, conceitos que são muito usados no estudo de métodos do tipo restrições ativas para problemas com restrições de caixa.

Definição 1.4. O conjunto dos índices das restrições ativas em $x$ é definido por

$$
\mathcal{A}(x)=\left\{i:[x]_{i}=[l]_{i} \text { ou }[x]_{i}=[u]_{i}\right\}
$$

As variáveis com indices em $\mathcal{A}(x)$ são ditas fixas e as variáveis com indices fora de $\mathcal{A}(x)$ são ditas livres. 
Definição 1.5. A face do conjunto factível $\Omega_{\square}$ que contém $x$ é dada por

$$
\left\{y \in \Omega_{\square}:[y]_{i}=[x]_{i} \text { se }[x]_{i} \in\left\{[l]_{i},[u]_{i}\right\}\right\} .
$$

Ou seja, a face do conjunto factível que contém um ponto $x$ pode ser definida em termos do conjunto ativo.

Na proposição 1.3, que será provada no Apêndice A, afirmamos que, de fato, podemos encontrar um minimizador aproximado $x$ do problema (1.1) em $\Omega_{\square}$.

Proposição 1.3. Considere $x^{*}$ o minimizador do problema (1.1). Se x está suficientemente perto de $x^{*}$ e $x$ pertence à mesma face que $x^{*}$, então $x$ é um minimizador aproximado do problema (1.1).

\subsection{Método Moré-Toraldo}

Nesta seção, descrevemos em detalhes o método usado para solucionar o problema de programação quadrática com restrições de caixa (1.1) para o caso em que $q$ é uma quadrática convexa, apresentado em Moré e Toraldo (1991). O método usa o método dos Gradientes Conjugados (veja, por exemplo, Nocedal e Wright (1999)) para explorar a face da região factível definida pelo iterando atual, e o método do Gradiente Projetado (veja, por exemplo, Nocedal e Wright (1999)) para mudar de face.

O método dos Gradientes Conjugados é um método iterativo para a solução numérica de sistemas lineares cujas matrizes são simétricas e definidas positivas, ou, equivalentemente, o método dos Gradientes Conjugados é uma técnica de minimização de funções quadráticas convexas irrestritas.

O método do Gradiente Projetado é um método de programação não linear para a resolução de problemas de otimização com restrições de igualdade e desigualdade.

Começamos descrevendo o uso do método dos Gradientes Conjugados. Dada a iteração $k$, um ponto $x_{k}$ e o conjunto ativo $\mathcal{A}\left(x_{k}\right)$, usamos o mé- 
todo dos Gradientes Conjugados para calcular uma solução aproximada do subproblema

$$
\begin{array}{ll}
\text { Minimizar } & q\left(x_{k}+d\right) \\
\text { sujeita a } & {[d]_{i}=0, \quad i \in \mathcal{A}\left(x_{k}\right) .}
\end{array}
$$

Este subproblema é um problema de programação quadrática irrestrito nas variáveis livres.

Note que, se $x_{k}$ está na mesma face de $x^{*}, x_{k}+d$ é a solução do problema (1.1), com $d$ minimizador de (1.9).

O método dos Gradientes Conjugados para a solução do subproblema (1.9) é implementado expressando este subproblema em termos de um subproblema equivalente usando apenas as variáveis livres.

Se $i_{1}, \ldots, i_{m_{k}}$ são os índices das variáveis livres e a matriz $Z_{k}$ é definida como uma matriz em $\mathbb{R}^{n \times m_{k}}$ cuja $j$-ésima coluna é a $i_{j}$-ésima coluna da matriz identidade em $\mathbb{R}^{n \times n}$, então o subproblema (1.9) é equivalente ao subproblema irrestrito

$$
\min \left\{q_{k}(w): w \in \mathbb{R}^{m_{k}}\right\}
$$

em que

$$
q_{k}(w) \equiv q\left(x_{k}+Z_{k} w\right)-q\left(x_{k}\right)
$$

Sejam a matriz $H_{k}$ e o vetor $r_{k}$, respectivamente, a matriz Hessiana e o gradiente reduzidos de $q$ em $x_{k}$ com respeito às variáveis livres. Se $H$ é a matriz Hessiana da quadrática $q$, então

$$
H_{k}=Z_{k}^{T} H Z_{k}, \quad r_{k}=Z_{k}^{T} \nabla q\left(x_{k}\right)
$$

Note que a matriz $H_{k}$ é obtida tomando as linhas e colunas de $H$ cujos índices correspondem às variáveis livres. Similarmente, $r_{k}$ é obtido de $\nabla q\left(x_{k}\right)$, tomando as componentes cujos índices correspondem às variáveis livres.

Propriedade 1.1. Seja q uma função quadrática. Sejam $H_{k}$ e $r_{k}$, respectivamente, a matriz Hessiana e o gradiente reduzidos de $q$ em $x_{k}$ com respeito às 
variáveis livres. Então,

$$
q\left(x_{k}+Z_{k} w\right)-q\left(x_{k}\right)=\frac{1}{2} w^{T} H_{k} w+r_{k}^{T} w
$$

Prova: De fato, como $q$ é quadrática

$$
q(x)=\frac{1}{2} x^{T} H x+x^{T} c \quad \text { e } \quad \nabla q(x)=H x+c
$$

com $H$ simétrica. Assim,

$$
\begin{aligned}
q\left(x_{k}+Z_{k} w\right)-q\left(x_{k}\right)= & \frac{1}{2}\left(x_{k}+Z_{k} w\right)^{T} H\left(x_{k}+Z_{k} w\right)+\left(x_{k}+Z_{k} w\right)^{T} c \\
& -\frac{1}{2} x_{k}^{T} H x_{k}-x_{k}^{T} c \\
= & w^{T} Z_{k}^{T} H x_{k}+\frac{1}{2} w^{T} Z_{k}^{T} H Z_{k} w+w^{T} Z_{k}^{T} c \\
= & w^{T} Z_{k}^{T}\left(H x_{k}+c\right)+\frac{1}{2} w^{T} Z_{k}^{T} H Z_{k} w \\
= & w^{T} Z_{k}^{T} \nabla q(x)+\frac{1}{2} w^{T} Z_{k}^{T} H Z_{k} w \\
= & \frac{1}{2} w^{T} H_{k} w+r_{k}^{T} w .
\end{aligned}
$$

Dado um ponto inicial $w_{0} \in \mathbb{R}^{m_{k}}$, o método dos Gradientes Conjugados gera uma sequência de iterandos $w_{0}, w_{1}, \ldots$ que termina na solução do problema (1.10) em, no máximo, $m_{k}$ iterações.

Executamos o método dos Gradientes Conjugados até que seja gerado um ponto $w_{j}$ de forma que

$$
q_{k}\left(w_{j-1}\right)-q_{k}\left(w_{j}\right) \leq \eta_{1} \max \left\{q_{k}\left(w_{l-1}\right)-q_{k}\left(w_{l}\right): 1 \leq l<j\right\},
$$

para alguma constante fixa $\eta_{1} \in(0,1)$.

A solução aproximada do problema (1.9) é, então, $d_{k}=Z_{k} w_{j_{k}}$, em que $j_{k}$ é o primeiro índice $j$ que satisfaz (1.12).

O critério de parada (1.12) detecta quando o método dos Gradientes Conjugados não obtém progresso suficiente. Uma das vantagens deste critério é 
que é invariante à escala, isto é, o critério é invariante se é aplicado à função $\alpha q$ para algum $\alpha>0$. Outra vantagem é que este critério em algum momento será satisfeito dado que a sequência $\left\{q_{k}\left(w_{j}\right)\right\}$ é monótona decrescente e $q_{k}$ é limitada inferiormente (o que acontece neste caso por $q$ ser uma função quadrática convexa).

Podem ser usados outros critérios, mas (1.12) é o apropriado para o método dos Gradientes Conjugados. Por exemplo, o critério $\left\|\nabla q_{k}\left(w_{j}\right)\right\|_{2} \leq \eta_{1}\left\|\nabla q_{k}(0)\right\|_{2}$, para algum $\eta_{1}>0$, é frequentemente usado ao invés de (1.12). Este critério é invariante à escala. Além disso, é, em algum momento, satisfeito, já que $w_{j}$ resolve o subproblema (1.10) para algum $j \leq m_{k}$. Por outro lado, o comportamento de $\left\|\nabla q_{k}\left(w_{j}\right)\right\|_{2}$ é, com frequência, errático.

Em geral, não temos $x_{k+1}=x_{k}+d_{k}$, pois isto pode produzir um $x_{k+1}$ inviável com relação a $\Omega_{\square}$. Alguns algoritmos usam $x_{k+1}=x_{k}+\alpha_{k} d_{k}$, em que $\alpha_{k}$ é o minimizador de $q\left(x_{k}+\alpha d_{k}\right)$ no intervalo $\left[0, \mu_{k}\right]$ e

$$
\mu_{k}=\max \left\{\alpha \geq 0: l \leq x_{k}+\alpha d_{k} \leq u\right\}
$$

Porém, nesta estratégia, somente uma restrição é usualmente adicionada a cada iteração, o que pode levar a um algoritmo ineficiente, já que, para obter convergência, pode-se precisar de um número grande de iterações. Usaremos uma busca projetada para definir $\alpha_{k}$. Assim

$$
x_{k+1}=P_{\Omega_{\square}}\left(x_{k}+\alpha_{k} d_{k}\right),
$$

em que $P_{\Omega_{\square}}$ é a projeção (1.6). A busca projetada será estudada em detalhes na Seção 1.1.1. Por agora, é apenas necessário notar que a busca projetada escolhe um $\alpha_{k}>0$ tal que $q\left(x_{k+1}\right)<q\left(x_{k}\right)$, e que mais de uma restrição pode ser adicionada ao conjunto ativo quando $\alpha_{k}>\mu_{k}$.

Se $x_{k+1}$, gerado pelo método dos Gradientes Conjugados, está na face que contém a solução do problema original, então a face continua sendo explorada. A decisão de continuar com o método dos Gradientes Conjugados é baseada na observação de se identificar se $x$ está na face que contém a solução. 
Definição 1.6. O conjunto identificador em $x$ é definido por

$$
\mathcal{B}(x)=\left\{i:[x]_{i}=[l]_{i} \text { e }[\nabla q(x)]_{i} \geq 0, \text { ou }[x]_{i}=[u]_{i} \text { e }[\nabla q(x)]_{i} \leq 0\right\} .
$$

Se o método dos Gradientes Conjugados produz um ponto $x_{k+1}$ tal que $\mathcal{B}\left(x_{k+1}\right)=\mathcal{A}\left(x_{k+1}\right)$, então continuamos explorando esta face com o método dos Gradientes Conjugados. A condição $\mathcal{B}\left(x_{k+1}\right)=\mathcal{A}\left(x_{k+1}\right)$ não garante que $x_{k+1}$ esteja na mesma face que contém a solução, mas, se $x_{k+1}$ não está na mesma face que contém a solução, a propriedade de terminação finita do método dos Gradientes Conjugados garante que, em algum momento, será gerado um iterando que viola a condição $\mathcal{B}\left(x_{k+1}\right)=\mathcal{A}\left(x_{k+1}\right)$.

Podemos continuar explorando a face atual, salvando o último iterando $w_{j}$ e a direção de busca $s_{j}$ gerada pelo método dos Gradientes Conjugados. Estabelecendo $w_{j}$ e $s_{j}$ como o ponto e a direção inicial da iteração seguinte, preservamos a informação e, assim, a propriedade de terminação finita do método dos Gradientes Conjugados.

Depois que o método dos Gradientes Conjugados explorou uma face, alguns métodos usam múltiplos critérios para escolher uma face diferente. Usaremos o método do Gradiente Projetado (veja, por exemplo, Nocedal e Wright (1999)).

Para o problema de programação quadrática (1.1), o método do Gradiente Projetado gera uma sequência $\left\{y_{j}\right\}$, sendo

$$
y_{j+1}=P_{\Omega_{\square}}\left(y_{j}-\alpha_{j} \nabla q\left(y_{j}\right)\right),
$$

em que $P_{\Omega_{\square}}$ é a projeção (1.6) e $\alpha_{j}>0$ é escolhido por uma busca projetada tal que $q\left(y_{j+1}\right)<q\left(y_{j}\right)$. Esta busca projetada será apresentada em detalhes na Seção 1.1.1.

O método do Gradiente Projetado é usado para selecionar uma nova face da seguinte maneira: dado $x_{k}$, geramos iterandos pelo método do Gradiente Projetado com $y_{0}=x_{k}$. Se, para alguma constante fixa $\eta_{2}$, quaisquer dos dois critérios

$$
\begin{gathered}
\mathcal{A}\left(y_{j}\right)=\mathcal{A}\left(y_{j-1}\right) \quad \text { ou } \\
q\left(y_{j-1}\right)-q\left(y_{j}\right) \leq \eta_{2} \max \left\{q\left(y_{l-1}\right)-q\left(y_{l}\right): 1 \leq l<j\right\}
\end{gathered}
$$


é satisfeito, então exploramos a face do conjunto factível que contém $y_{j_{k}}$, sendo $j_{k}$ o primeiro índice que satisfaz (1.15) ou (1.16).

O Algoritmo GPCG (nomenclatura tomada de Moré e Toraldo (1991), baseada na sigla para Gradient Projection Conjugate Gradient) descreve por completo o método para encontrar a solução para o problema (1.1).

\section{Algoritmo 1.1 GPCG}

Dados um ponto $x_{0}$ e os escalares $\eta_{1}>0, \eta_{2}>0, \epsilon>0, \tau>0$.

Passo 1: Faça $k \leftarrow 0$.

Passo 2: Faça $j \leftarrow 0, y_{j} \leftarrow x_{k}$.

Passo 3: Gere iterações do Gradiente Projetado $y_{1}, y_{2}, \ldots y_{j_{k}}$, sendo $j_{k} \mathrm{O}$ primeiro índice que satisfaz (1.15) ou (1.16). Faça $x_{k} \leftarrow y_{j_{k}}$. Se $\left\|\nabla_{\Omega_{\square}} q\left(x_{k}\right)\right\|_{2} \leq \tau\left\|\nabla q\left(x_{0}\right)\right\|_{2}$, pare e devolva $x_{k}$ como solução.

Passo 4: Faça $j \leftarrow 0, w_{0} \leftarrow 0$.

Passo 5: Gere iterações dos Gradientes Conjugados $w_{1}, w_{2}, \ldots w_{j_{k}}$, sendo $j_{k} \mathrm{O}$ primeiro índice que satisfaz (1.12). Faça $d_{k} \leftarrow Z_{k} w_{j_{k}}$.

Passo 6: Calcule $\alpha_{k}$ usando busca projetada para definir $x_{k+1}$ por (1.14). Se $\left\|\nabla_{\Omega_{\square}} q\left(x_{k+1}\right)\right\|_{2} \leq \tau\left\|\nabla q\left(x_{0}\right)\right\|_{2}$, pare e devolva $x_{k}$ como solução.

Passo 7: Se $\mathcal{B}\left(x_{k+1}\right)=\mathcal{A}\left(x_{k+1}\right)$ então Se $\left\|r_{k}\right\|_{2} \leq \epsilon$ pare com o iterando atual como solução.

Senão, volte para o Passo 5.

Passo 8: Faça $k \leftarrow k+1$, e volte ao Passo 2.

\subsubsection{Busca projetada}

A busca projetada no algoritmo GPCG requer um $\alpha_{k}>0$ que produza um decréscimo suficiente na função $\phi_{k}: \mathbb{R} \rightarrow \mathbb{R}$ definida por

$$
\phi_{k}(\alpha)=q\left(P_{\Omega_{\square}}\left(x_{k}+\alpha d_{k}\right)\right)
$$



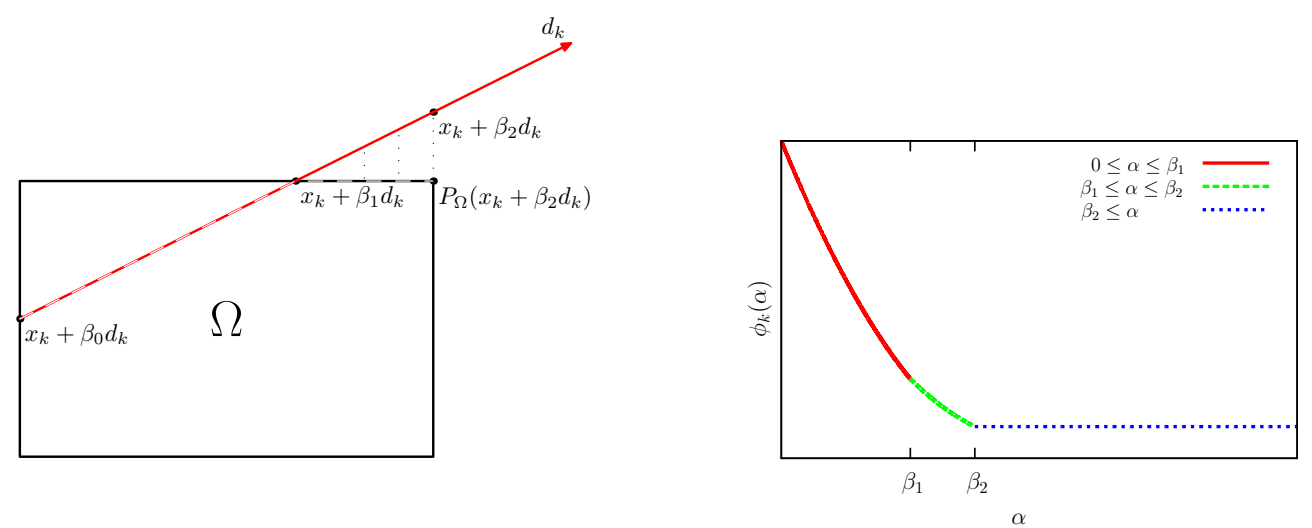

Figura 1.1: Pontos de interrupção de $\phi_{k}$

em que $P_{\Omega_{\square}}$ é a projeção (1.6) e $d_{k}$ é a direção de busca.

A condição de decréscimo suficiente requer que $\alpha_{k}>0$ satisfaça

$$
\phi_{k}\left(\alpha_{k}\right) \leq \phi_{k}(0)+\mu \nabla q\left(x_{k}\right)^{T}\left(P_{\Omega_{\square}}\left(x_{k}+\alpha_{k} d_{k}\right)-x_{k}\right),
$$

para $\mu \in\left(0, \frac{1}{2}\right)$. A condição de decréscimo suficiente pode ser satisfeita para as duas escolhas de $d_{k}$ no algoritmo GPCG. Note que, no método Gradiente Projetado, $d_{k}=-\nabla q\left(x_{k}\right)$, enquanto que, para o método dos Gradientes Conjugados, $d_{k}=Z_{k} w_{j_{k}}$, sendo $w_{j_{k}}$ produzido pelo método dos Gradientes Conjugados para o subproblema (1.10).

Observe que $\phi_{k}$ é contínua e quadrática por partes, devido a $P_{\Omega_{\square}}\left(x_{k}+\alpha d_{k}\right)$ ser uma função linear de $\alpha$ em qualquer intervalo em que o conjunto ativo de $P_{\Omega_{\square}}\left(x_{k}+\alpha d_{k}\right)$ é inalterado. Como $q$ é estritamente convexa, $\phi_{k}$ é estritamente convexa por partes. Veja um exemplo na Figura 1.1.

Definimos os pontos de interrupção de $\phi_{k}$, com base em

$$
0=\beta_{0}<\beta_{1}<\ldots<\beta_{p}<\beta_{p+1}=+\infty
$$

de modo que $\mathcal{A}\left(P_{\Omega_{\square}}\left(x_{k}+\alpha d_{k}\right)\right)$ é constante para $\alpha \in\left(\beta_{i}, \beta_{i+1}\right)$ para $i=0, \ldots, p$. Permitimos valores infinitos para $[l]_{i}$ ou $[u]_{i}$ e, assim, podemos ter $p=0$ na definição dos pontos de interrupção. 
Quando $d_{k}=Z_{k} w_{j_{k}}$, a escolha de $Z_{k}$ garante que $x_{k}+\alpha d_{k}$ é factível para $\alpha$ em $\left[0, \beta_{1}\right]$ e, assim, a condição (1.17) pode ser satisfeita, para todo $\alpha_{k}>0$ suficientemente pequeno, se $\phi_{k}^{\prime}(0)<0$. Note que, para $d_{k}=Z_{k} w_{j_{k}}, \beta_{1}$ coincide com $\mu_{k}=\max \left\{\alpha \geq 0: l \leq x_{k}+\alpha d_{k} \leq u\right\}$ (definido em (1.13)). Por isso, se $r_{k}=Z_{k}^{T} \nabla q\left(x_{k}\right)$ é o gradiente reduzido (definido em (1.11)), então

$$
\phi_{k}^{\prime}(0)=\nabla q\left(x_{k}\right)^{T} d_{k}=r_{k}^{T} w_{j_{k}} .
$$

Como $w_{j_{k}}$ é uma direção de descida no método dos Gradientes Conjugados, temos que $\phi_{k}^{\prime}(0)<0$, a menos que $r_{k}=0$.

Quando $d_{k}=-\nabla q\left(x_{k}\right)$, temos uma situação similar. Digamos que $r_{k}$ é o gradiente projetado descontínuo $\nabla_{\Omega_{\square}} q\left(x_{k}\right)$. Lembremos que, pela Definição 1.3,

$$
\left[\nabla_{\Omega_{\square}} q(x)\right]_{i}= \begin{cases}{[\nabla q(x)]_{i},} & \text { se }[x]_{i} \in\left([l]_{i},[u]_{i}\right), \\ \min \left\{[\nabla q(x)]_{i}, 0\right\}, & \text { se }[x]_{i}=[l]_{i}, \\ \max \left\{[\nabla q(x)]_{i}, 0\right\}, & \text { se }[x]_{i}=[u]_{i} .\end{cases}
$$

Então, $P_{\Omega_{\square}}\left(x_{k}-\alpha \nabla q\left(x_{k}\right)\right)=x_{k}-\alpha r_{k}$, para $\alpha \in\left[0, \beta_{1}\right]$, e

$$
\phi_{k}(\alpha)=q\left(P_{\Omega_{\square}}\left(x_{k}-\alpha \nabla q\left(x_{k}\right)\right)\right)=q\left(x_{k}-\alpha r_{k}\right), \quad \alpha \in\left[0, \beta_{1}\right] .
$$

Como $\nabla q\left(x_{k}\right)^{T} r_{k}=\left\|r_{k}\right\|_{2}^{2}>0$, a menos que $x_{k}$ seja a solução, a condição (1.17) pode ser satisfeita para todo $\alpha$ suficientemente pequeno. Note que, se $x_{k}$ está na borda de $\Omega_{\square}, x_{k}+\alpha d_{k}$ pode ser inviável para qualquer $\alpha>0$. Nesse caso, $\mathcal{A}\left(P_{\Omega_{\square}}\left(x_{k}+\alpha d_{k}\right)\right)=\mathcal{B}\left(x_{k}\right)$, para $\alpha$ em $\left(0, \beta_{1}\right)$.

O algoritmo da busca projetada gera uma sequência decrescente $\left\{\alpha_{k}^{(l)}\right\}$ de valores positivos tais que

$$
\alpha_{k}^{(l+1)} \in\left[\gamma_{1} \alpha_{k}^{(l)}, \gamma_{2} \alpha_{k}^{(l)}\right], \quad 0<\gamma_{1}<\gamma_{2}<1
$$

Escolhemos um $\alpha_{k}^{(0)}>0$ e estabelecemos $\alpha_{k}$ como o primeiro valor que satisfaça a condição de decréscimo suficiente. Uma das vantagens deste processo é que $\alpha_{k}$ é encontrado em um número finito de passos.

O valor inicial testado $\alpha_{k}^{(0)}$ é o minimizador da função quadrática que re- 
presenta $\phi_{k}$ em $\left[0, \beta_{1}\right]$. Assim, afirmamos a seguinte propriedade.

Propriedade 1.2. Seja $k$ a iteração atual e $\phi_{k}: \mathbb{R} \rightarrow \mathbb{R}$ uma função definida por $\phi_{k}(\alpha)=q\left(P_{\Omega_{\square}}\left(x_{k}+\alpha d_{k}\right)\right)$. Então

$$
\alpha_{k}^{(0)}=-\frac{\phi_{k}^{\prime}(0)}{\phi_{k}^{\prime \prime}(0)}
$$

Se $d_{k}=-\nabla q\left(x_{k}\right)$, ent $\tilde{a} O$

$$
\alpha_{k}^{(0)}=\frac{\left\|r_{k}\right\|_{2}^{2}}{r_{k}^{T} H r_{k}}
$$

e, no caso $d_{k}=Z_{k} w_{j_{k}}$, temos que

$$
\alpha_{k}^{(0)}=-\frac{r_{k}^{T} w_{j_{k}}}{\left(w_{j_{k}}\right)^{T} H_{k} w_{j_{k}}}=1 .
$$

Para a prova da Propriedade 1.2, veja o Apêndice A. Se $\alpha_{k}^{(0)}<\beta_{1}$, para cada escolha de $d_{k}$, então $\alpha_{k}^{(0)}$ satisfaz a condição de decréscimo suficiente com $\mu \leq \frac{1}{2}$, e, assim, $\alpha_{k}^{(0)}$ torna-se $\alpha_{k}$.

Dado um valor inicial $\alpha_{k}^{(0)}$, calculamos $\alpha_{k}^{*}$, minimizador da quadrática $\phi_{q}\left(\alpha_{k}\right)=\left(\frac{\phi_{k}\left(\alpha_{k}^{(0)}\right)-\phi_{k}(0)-\alpha_{k}^{(0)} \phi_{k}^{\prime}(0)}{\left(\alpha_{k}^{(0)}\right)^{2}}\right) \alpha_{k}^{2}+\phi_{k}^{\prime}(0) \alpha_{k}+\phi_{k}(0)$, que interpola $\phi_{k}(0)$, $\phi_{k}^{\prime}(0), \phi_{k}\left(\alpha_{k}\right)$ e usamos $\bar{\alpha}_{k}$ como uma nova estimativa, sendo

$$
\bar{\alpha}_{k}=\left\{\begin{aligned}
\frac{1}{100} \alpha_{k}, & \text { se } \alpha_{k}^{*}<\frac{1}{100} \alpha_{k} \\
\alpha_{k}^{*}, & \text { se } \frac{1}{100} \alpha_{k} \leq \alpha_{k}^{*} \leq \frac{1}{2} \alpha_{k} \\
\frac{1}{2} \alpha_{k}, & \text { se } \alpha_{k}^{*}>\frac{1}{2} \alpha_{k} .
\end{aligned}\right.
$$

Veja, a seguir, o algoritmo da busca projetada.

\section{Algoritmo 1.2 Busca projetada}

$\operatorname{Dados} \alpha_{k}^{(0)}$ e $\mu \in\left(0, \frac{1}{2}\right)$ 
Passo 1: Faça $\alpha_{k} \leftarrow \alpha_{k}^{(0)}$.

Passo 2: Se $\phi_{k}\left(\alpha_{k}\right) \leq \phi(0)+\mu \alpha_{k} \phi_{k}^{\prime}(0)$ então pare e devolva $\alpha_{k}$ como solução.

$$
\text { Senão faça } \alpha_{k}^{*}=-\frac{\phi_{k}^{\prime}(0) \alpha_{k}^{2}}{2\left(\phi_{k}\left(\alpha_{k}\right)-\phi(0)-\alpha_{k} \phi_{k}^{\prime}(0)\right)} \text {. }
$$

Passo 3: Se $\alpha_{k}^{*}<\frac{1}{100} \alpha_{k}$, então faça $\alpha_{k}^{*} \leftarrow \frac{1}{100} \alpha_{k}$.

Passo 4: Se $\alpha_{k}^{*}>\frac{1}{2} \alpha_{k}$, então faça $\alpha_{k}^{*} \leftarrow \frac{1}{2} \alpha_{k}$.

Passo 5: Faça $\alpha_{k} \leftarrow \alpha_{k}^{*}$ e volte ao Passo 2 .

\subsubsection{Resultados de convergência}

Os principais resultados teóricos para o algoritmo GPCG são que ele converge para a solução quando aplicado a problemas estritamente convexos, e que, se o problema é não degenerado, no sentido que $\left[\nabla q\left(x^{*}\right)\right]_{i} \neq 0, i \in \mathcal{A}\left(x^{*}\right)$, GPCG termina em um número finito de iterações.

Os seguintes teoremas são enunciados e provados em Calamai e Moré (1987) e servem como uma ferramenta para as provas dos teoremas de convergência.

Teorema 1.3. Seja $q: \mathbb{R}^{n} \rightarrow \mathbb{R}$ continuamente diferenciável em $\Omega_{\square}$ e seja $\left\{x_{k}\right\}$ a sequência gerada pelo método do Gradiente Projetado.

Suponha $\mathcal{K}_{G P}=\left\{k: x_{k}\right.$ é gerado pelo método do Gradiente Projetado $\}$ infinito. Se alguma subsequência $\left\{x_{k}: k \in K\right\}$, com $K \subset \mathcal{K}_{G P}$, é limitada, então

$$
\lim _{k \rightarrow \infty}\left\|\nabla_{\Omega_{\square}} q\left(x_{k+1}\right)\right\|_{2}=0, \quad k \in K .
$$

Ainda mais, qualquer ponto limite de $\left\{x_{k}: k \in \mathcal{K}_{G P}\right\}$ é um ponto estacionário do problema (1.1).

Prova: Ver Teorema 5.2 de Calamai e Moré (1987).

Teorema 1.4. Seja $q: \mathbb{R}^{n} \rightarrow \mathbb{R}$ uma função continuamente diferenciável em $\Omega_{\square}$ e seja $\left\{x_{k}\right\}$ uma sequência arbitrária que converge ao ponto $x^{*}$. Se $\left\{\left\|\nabla_{\Omega_{\square}} q\left(x_{k}\right)\right\|_{2}\right\}$ converge a zero e $x^{*}$ é nãa degenerado, então $\mathcal{A}\left(x_{k}\right)=\mathcal{A}\left(x^{*}\right)$, para algum $k$ suficientemente grande. 
Prova: Ver Teorema 4.1 de Calamai e Moré (1987).

Vejamos agora os teoremas de convergência do método GPCG, que aparecem em Moré e Toraldo (1991) e repetimos aqui por completude.

Teorema 1.5. (Teorema 5.1 de Moré e Toraldo (1991)) Seja $q: \mathbb{R}^{n} \rightarrow \mathbb{R}$ uma função quadrática estritamente convexa. Se $\left\{x_{k}\right\}$ é a sequência gerada pelo algoritmo GPCG para o problema (1.1), então $\left\{x_{k}\right\}$ termina em $x^{*}$ solução do problema (1.1) em um número finito de passos ou $\left\{x_{k}\right\}$ converge a $x^{*}$.

Prova: Note que, se GPCG termina em um número finito de passos, então termina em ponto estacionário do problema (1.1). Como $q$ é estritamente convexa, o problema (1.1) tem um único ponto estacionário, que é a solução $x^{*}$.

Considere agora o caso em que GPCG gere uma sequência infinita $\left\{x_{k}\right\}$. Como $q$ é uma função quadrática estritamente convexa e $\left\{q\left(x_{k}\right)\right\}$ é uma sequência decrescente, podemos afirmar que a sequência $\left\{x_{k}\right\}$ é limitada. Em particular, $\mathcal{K}_{G P}$ é infinito e $\left\{x_{k}: k \in \mathcal{K}_{G P}\right\}$ é limitada. Assim, pelo Teorema 1.3, afirmamos que existe uma subsequência de $\left\{x_{k}\right\}$ que converge a $x^{*}$, e, assim, $\left\{q\left(x_{k}\right)\right\}$ converge a $q\left(x^{*}\right)$.

Agora verifiquemos que $\left\{x_{k}\right\}$ converge a $x^{*}$ como desejamos:

$$
\begin{aligned}
q\left(x_{k}\right)-q\left(x^{*}\right) & =\frac{1}{2} x_{k}^{T} H x_{k}-x_{k}^{T} c-\frac{1}{2} x^{* T} H x^{*}+x^{* T} c-x_{k}^{T} H x^{*}+x_{k}^{T} H x^{*} \\
& =\left(H x^{*}-c\right)^{T} x_{k}-\left(H x^{*}-c\right)^{T} x^{*}+\frac{1}{2}\left(x_{k}-x^{*}\right)^{T} H\left(x_{k}-x^{*}\right) \\
& =\nabla q\left(x^{*}\right)^{T}\left(x_{k}-x^{*}\right)+\frac{1}{2}\left(x_{k}-x^{*}\right)^{T} H\left(x_{k}-x^{*}\right) \\
& \geq \frac{1}{2}\left(x_{k}-x^{*}\right)^{T} H\left(x_{k}-x^{*}\right) \\
& \geq \frac{\gamma}{2}\left(x_{k}-x^{*}\right)^{T}\left(x_{k}-x^{*}\right)
\end{aligned}
$$

sendo $\gamma$ o menor autovalor da matriz Hessiana de $q$.

Assim, $q\left(x_{k}\right)-q\left(x^{*}\right) \geq \frac{\gamma}{2}\left\|x_{k}-x^{*}\right\|_{2}^{2}$ e, como $\left\{q\left(x_{k}\right)\right\}$ converge a $q\left(x^{*}\right),\left\{x_{k}\right\}$ converge a $x^{*}$. 
O Teorema 1.5 não usa fortemente as propriedades do método dos Gradientes Conjugados. A convergência do GPCG depende da propriedade de convergência do método Gradiente Projetado, e o resultado que o método dos Gradientes Conjugados produz iterações tais que $q\left(x_{k+1}\right) \leq q\left(x_{k}\right)$.

Agora mostramos que a terminação finita do GPCG depende da propriedade de terminação finita do método dos Gradientes Conjugados nos subproblemas e na propriedade de identificação do Gradiente Projetado.

Teorema 1.6. (Teorema 5.2 de Moré e Toraldo (1991)) Seja $q: \mathbb{R}^{n} \rightarrow \mathbb{R}$ uma função quadrática estritamente convexa. Se a solução $x^{*}$ do problema (1.1) é não degenerada, no sentido que

$$
\left[\nabla q\left(x^{*}\right)\right]_{i} \neq 0, \quad i \in \mathcal{A}\left(x^{*}\right)
$$

então o algoritmo GPCG termina na solução $x^{*}$ em um número finito de passos.

Prova: Afirmamos que, se o método dos Gradientes Conjugados no algoritmo GPCG gera um ponto $x_{k} \operatorname{com} \mathcal{A}\left(x_{k}\right)=\mathcal{A}\left(x^{*}\right)$ e suficientemente perto de $x^{*}$, então o método dos Gradientes Conjugados produz a solução $x^{*}$ em um número finito de passos.

Estabelecemos esta afirmação, notando que, como o método dos Gradientes Conjugados não viola nenhuma restrição ativa, então

$$
\mathcal{A}\left(x^{*}\right)=\mathcal{A}\left(x_{k}\right) \subset \mathcal{A}\left(x_{k+1}\right) .
$$

Como $\mathcal{A}(x) \subset \mathcal{A}\left(x^{*}\right)$, para qualquer $x$ suficientemente perto de $x^{*}$, mostramos que $\mathcal{A}\left(x_{k+1}\right)=\mathcal{A}\left(x^{*}\right)$.

Agora considere qualquer $x$ suficientemente próximo de $x^{*}$. Pela continuidade de $\nabla q$, a suposição de que $x^{*}$ é não degenerado e que $\mathcal{A}\left(x_{k}\right)=\mathcal{A}\left(x^{*}\right)$, temos que $\mathcal{B}(x)=\mathcal{A}\left(x^{*}\right)=\mathcal{A}(x)$.

Em particular $\mathcal{B}\left(x_{k+1}\right)=\mathcal{A}\left(x_{k+1}\right)$, já que $\mathcal{A}\left(x_{k+1}\right)=\mathcal{A}\left(x^{*}\right)$. Isto implica que o método dos Gradientes Conjugados continua explorando a face atual e 
produz a solução $x^{*}$ em um número finito de passos.

Resta mostrar que o ponto $x_{k}$ produzido pelo GPCG é gerado em um número finito de passos.

Sendo $\mathcal{K}_{G P}$ o conjunto de índices da sequência gerada, como nos Teoremas 1.3 e 1.4 , então $\mathcal{A}\left(y_{j_{k}}\right)=\mathcal{A}\left(x^{*}\right)$, em que $x_{k}$ é suficientemente próximo de $x^{*}$ e $k \in \mathcal{K}_{G P}$.

Assim, o método dos Gradientes Conjugados no GPCG em algum momento gera um ponto $x_{k} \operatorname{com} \mathcal{A}\left(x_{k}\right)=\mathcal{A}\left(x^{*}\right)$ e suficientemente próximo de $x^{*}$.

\subsubsection{Resultados numéricos}

Nesta seção, apresentamos os resultados numéricos obtidos ao usar o método Moré-Toraldo para resolver os três problemas apresentados em Moré e Toraldo (1991): Obstacle, Journal Bearing e Torsion.

Implementamos o método Moré-Toraldo em Fortran 77 e compilamos em gfortran, usando o sistema operacional Ubuntu 11.10. Daqui em diante, chamaremos nossa implementação de GPCG. Os experimentos numéricos foram realizados em um PC com Processador 2a Geração Intel Core i3 2310M, 2.1 GHz, 4GB de memória.

Os resultados foram obtidos com os seguintes parâmetros:

$$
\eta_{1}=0.1, \quad \eta_{2}=0.25, \quad \mu=0.1, \quad \tau=10^{-5}, \quad \epsilon=10^{-8} .
$$

Lembrando que $\eta_{1}$ aparece no critério de parada do método dos Gradientes Conjugados (1.12), $\eta_{2}$ no critério de parada do método do Gradiente Projetado (1.16), $\mu$ na condição de decréscimo suficiente da busca projetada (1.17), $\tau$ no critério de parada (1.7) e $\epsilon$ no Passo 7 do Algoritmo GPCG.

Os três problemas para os experimentos numéricos foram obtidos da coleção CUTEr Bongartz et al. (1995). Como suas Hessianas não são definidas 
positivas, optamos por resolver o problema

$$
\begin{array}{ll}
\text { Minimizar } & \frac{1}{2} x^{T}(H+\rho I) x+b^{T} x \\
\text { sujeita a } & x \in \Omega_{\square},
\end{array}
$$

com $\rho$ tal que $(H+\rho I)$ é definida positiva. Nos três problemas, $\rho=10^{-8}$.

Os resultados numéricos para o problema Journal Bearing foram obtidos considerando o problema equivalente

$$
\min \{\bar{q}(v): v \geq 0\}, \quad \bar{q}(v)=q\left(D^{-1} v\right),
$$

sendo $D$ uma matriz diagonal, tal que a matriz Hessiana de $\bar{q}$ tenha entradas unitárias na diagonal, como feito em Moré e Toraldo (1991).

Os resultados obtidos pela aplicação do GPCG para a resolução dos três problemas da coleção CUTEr, bem como os dados fornecidos no artigo Moré e Toraldo (1991), podem ser encontrados nas Tabelas 1.1-1.7. Nestas tabelas, GPCG denota a implementação da aluna, Moré-Toraldo denota os resultados apresentados em Moré e Toraldo (1991), $n$ é o número de variáveis do problema, $n$ free é o número de variáveis livres no ponto final $x^{*}$ obtido pelo método, $k$ é o número de iterações, $n_{f g}$ é o número de avaliações da função-gradiente e $n_{h}$ é o número de produtos Hessiana-vetor.

Nas Tabelas 1.1-1.7, $x_{0}$ é igual a $l$, se o ponto inicial é o limitante inferior; igual a $u$, se o ponto inicial é o limitante superior; igual a $m^{*}$, se o ponto inicial é o vetor $\frac{1}{2}(l+u)$; igual a $e$, se o ponto inicial é o vetor com todas suas componentes iguais à unidade; igual a 0 , se o ponto inicial é o vetor com todas suas componentes iguais a zero.

Tanto nos resultados apresentados em Moré e Toraldo (1991), como nos resultados da nossa implementação, para todos os problemas o método parou atendendo ao critério de convergência. Ao comparar esses resultados, pode-se ver que a principal diferença está no número de variáveis livres. Isso pode ser devido à implementação dos problemas ou ao tratamento de erros numéricos. Note que existe diferença entre os problemas tomados da coleção CUTEr e os problemas resolvidos em Moré e Toraldo (1991), principalmente no número de 
Tabela 1.1: Obstacle problem A

\begin{tabular}{|c|c|c|c|c|c|c|c|c|c|}
\hline & & \multicolumn{4}{|c|}{ GPCG } & \multicolumn{4}{|c|}{ Moré-Toraldo } \\
\hline$n$ & $x_{0}$ & nfree & $k$ & $n_{f g} / k$ & $n_{h} / k$ & nfree & $k$ & $n_{f g} / k$ & $n_{h} / k$ \\
\hline 5625 & $l$ & 2902 & 9 & 4.0 & 12.2 & 2122 & 9 & 4.1 & 13.2 \\
\hline 5625 & $e$ & 2895 & 9 & 4.0 & 13.4 & 2122 & 11 & 5.6 & 13.3 \\
\hline 10000 & $l$ & 5130 & 11 & 4.1 & 13.4 & 3843 & 10 & 4.5 & 15.7 \\
\hline 10000 & $e$ & 5127 & 10 & 6.3 & 19.6 & 3843 & 12 & 4.1 & 17.6 \\
\hline 15625 & $l$ & 7948 & 12 & 4.1 & 15.2 & 6108 & 11 & 4.3 & 17.1 \\
\hline 15625 & $e$ & 7939 & 14 & 4.4 & 17.9 & 6108 & 14 & 3.8 & 17.0 \\
\hline
\end{tabular}

Tabela 1.2: Obstacle problem B

\begin{tabular}{|c|c|c|c|c|c|c|c|c|c|}
\hline & & \multicolumn{4}{|c|}{ GPCG } & \multicolumn{4}{|c|}{ Moré-Toraldo } \\
\hline$n$ & $x_{0}$ & nfree & $k$ & $n_{f g} / k$ & $n_{h} / k$ & nfree & $k$ & $n_{f g} / k$ & $n_{h} / k$ \\
\hline 5625 & $l$ & 3843 & 8 & 4.0 & $\overline{13.6}$ & 4171 & 9 & 4.2 & 14.5 \\
\hline 5625 & $u$ & 3842 & 8 & 4.3 & 14.0 & 4171 & 8 & 4.2 & 15.0 \\
\hline 5625 & $m^{*}$ & 3843 & 6 & 4.2 & 12.8 & 4171 & 5 & 4.8 & 18.0 \\
\hline 10000 & $l$ & 7056 & 9 & 4.1 & 17.8 & 7588 & 8 & 4.3 & 21.7 \\
\hline 10000 & $u$ & 7057 & 10 & 4.3 & 14.5 & 7588 & 10 & 4.5 & 16.8 \\
\hline 10000 & $m^{*}$ & 7056 & 8 & 4.9 & 14.5 & 7588 & 7 & 4.2 & 18.2 \\
\hline 15625 & $l$ & 11318 & 10 & 5.0 & 19.9 & 11990 & 12 & 3.9 & 19.5 \\
\hline 15625 & $u$ & 11317 & 10 & 4.0 & 16.9 & 11990 & 11 & 4.4 & 19.6 \\
\hline 15625 & $m^{*}$ & 11317 & 7 & 4.7 & 20.4 & 11990 & 8 & 4.2 & 22.1 \\
\hline
\end{tabular}

variáveis dos problemas apresentados nas tabelas 1.3, 1.4 e 1.5. Mas, em geral, os resultados obtidos por GPCG estão próximos dos reportados em Moré e Toraldo (1991), o que nos leva a concluir que a implementação de GPCG está correta.

Tabela 1.3: Elastic-plastic torsion problem, 1 e 2

\begin{tabular}{|c|r|r|r|r|r|r|r|r|r|r|}
\hline & \multicolumn{6}{|c|}{ GPCG } & \multicolumn{5}{|c|}{ Moré-Toraldo } \\
\hline$x_{0}$ & $n$ & $n f r e e$ & $k$ & $n_{f g} / k$ & $n_{h} / k$ & $n$ & $n f r e e$ & $k$ & $n_{f g} / k$ & $n_{h} / k$ \\
\hline 0 & 5476 & 3560 & 8 & 9.6 & 24.8 & 5625 & 3953 & 9 & 4.4 & 27.1 \\
\hline$u$ & 5476 & 3560 & 11 & 4.6 & 15.6 & 5625 & 3953 & 11 & 4.5 & 20.1 \\
\hline 0 & 10000 & 6620 & 10 & 5.0 & 28.1 & 10000 & 7016 & 11 & 4.9 & 27.9 \\
\hline$u$ & 10000 & 6620 & 13 & 4.8 & 19.8 & 10000 & 7016 & 14 & 4.5 & 23.3 \\
\hline 0 & 14884 & 9984 & 11 & 7.0 & 30.2 & 15625 & 10961 & 12 & 5.0 & 33.5 \\
\hline$u$ & 14884 & 9984 & 14 & 4.8 & 22.2 & 15625 & 10961 & 14 & 5.0 & 27.5 \\
\hline
\end{tabular}


Tabela 1.4: Elastic-plastic torsion problem, 3 e 4.

\begin{tabular}{|c|r|r|r|r|r|r|r|r|r|r|}
\hline & \multicolumn{5}{|c|}{ GPCG } & \multicolumn{5}{|c|}{ Moré-Toraldo } \\
\hline$x_{0}$ & $n$ & $n$ free & $k$ & $n_{f g} / k$ & $n_{h} / k$ & $n$ & $n$ free & $k$ & $n_{f g} / k$ & $n_{h} / k$ \\
\hline 0 & 5476 & 1800 & 7 & 6.6 & 15.9 & 5625 & 2073 & 8 & 4.2 & 17.5 \\
\hline$u$ & 5476 & 1800 & 6 & 6.3 & 13.7 & 5625 & 2073 & 7 & 4.4 & 15.8 \\
\hline 0 & 10000 & 3476 & 8 & 7.1 & 17.6 & 10000 & 3632 & 8 & 4.5 & 21.8 \\
\hline$u$ & 10000 & 3476 & 8 & 5.5 & 15.8 & 10000 & 3632 & 7 & 4.7 & 19.8 \\
\hline 0 & 14884 & 5208 & 9 & 5.9 & 20.7 & 15625 & 5789 & 10 & 4.5 & 21.7 \\
\hline$u$ & 14884 & 5208 & 9 & 5.4 & 15.6 & 15625 & 5789 & 9 & 4.4 & 18.5 \\
\hline
\end{tabular}

Tabela 1.5: Elastic-plastic torsion problem, 5 e 6.

\begin{tabular}{|c|r|r|r|r|r|r|r|r|r|r|}
\hline & \multicolumn{4}{|c|}{ GPCG } & \multicolumn{5}{|c|}{ Moré-Toraldo } \\
\hline$x_{0}$ & $n$ & $n$ free & $k$ & $n_{f g} / k$ & $n_{h} / k$ & $n$ & $n$ free & $k$ & $n_{f g} / k$ & $n_{h} / k$ \\
\hline 0 & 5476 & 952 & 5 & 4.2 & 10.8 & 5625 & 1025 & 6 & 3.8 & 11.1 \\
\hline$u$ & 5476 & 952 & 4 & 6.5 & 8.8 & 5625 & 1025 & 5 & 4.0 & 10.0 \\
\hline 0 & 10000 & 1708 & 6 & 5.3 & 13.7 & 10000 & 1786 & 6 & 4.0 & 13.6 \\
\hline$u$ & 10000 & 1708 & 5 & 5.2 & 10.2 & 10000 & 1786 & 5 & 4.2 & 11.8 \\
\hline 0 & 14884 & 2568 & 7 & 5.3 & 15.0 & 15625 & 9248 & 6 & 4.5 & 18.8 \\
\hline$u$ & 14884 & 2568 & 6 & 5.3 & 11.8 & 15625 & 9248 & 5 & 4.8 & 14.6 \\
\hline
\end{tabular}

Tabela 1.6: Journal bearing problem A

\begin{tabular}{|c|r|r|r|r|r|r|r|r|r|}
\hline \multicolumn{2}{|c|}{} & \multicolumn{4}{|c|}{ GPCG } & \multicolumn{4}{|c|}{ Moré-Toraldo } \\
\hline$x_{0}$ & $n$ & $n$ free & $k$ & $n_{f g} / k$ & $n_{h} / k$ & $n$ free & $k$ & $n_{f g} / k$ & $n_{h} / k$ \\
\hline 0 & 5625 & 3528 & 9 & 5.6 & 20.7 & 3808 & 9 & 4.3 & 28.2 \\
\hline 0 & 10000 & 6359 & 10 & 7.1 & 25.1 & 6768 & 10 & 4.6 & 33.3 \\
\hline 0 & 15625 & 9968 & 10 & 8.5 & 30.3 & 10564 & 10 & 5.2 & 41.5 \\
\hline
\end{tabular}

Tabela 1.7: Journal bearing problem B

\begin{tabular}{|c|r|r|r|r|r|r|r|r|r|}
\hline \multicolumn{2}{|c|}{} & \multicolumn{5}{|c|}{ GPCG } & \multicolumn{4}{c|}{ Moré-Toraldo } \\
\hline$x_{0}$ & $n$ & $n$ nree & $k$ & $n_{f g} / k$ & $n_{h} / k$ & nfree & $k$ & $n_{f g} / k$ & $n_{h} / k$ \\
\hline 0 & 5625 & 2989 & 4 & 11.5 & 23.0 & 3359 & 5 & 5.0 & 31.6 \\
\hline 0 & 10000 & 5383 & 4 & 16.3 & 29.5 & 6040 & 7 & 4.7 & 31.0 \\
\hline 0 & 15625 & 8477 & 5 & 13.2 & 30.6 & 9426 & 7 & 5.1 & 40.2 \\
\hline
\end{tabular}




\section{2 "Método" NQC}

Durante o pós-doutorado da orientadora, Profa. Dra. Marina Andretta, em parceria com os professores José Mario Martínez (IMECC-Unicamp) e Mikhail Solodov (IMPA), foi desenvolvido e implementado o "método" NQC, usado para solucionar problemas de programação quadrática convexa com restrições de caixa. NQC é sigla para Newton para Quadráticas convexas em Caixas. Escrevemos "método" pois ele não possui convergência garantida (de fato, há problemas para o qual este procedimento não converge), mas ele se mostra muito eficiente na prática.

Lembrando que o problema a ser resolvido é o seguinte:

$$
\begin{array}{ll}
\text { Minimizar } & q(x) \\
\text { sujeita a } & x \in \Omega_{\square},
\end{array}
$$

onde $q: \mathbb{R}^{n} \rightarrow \mathbb{R}$ é uma função quadrática e

$$
\Omega_{\square}=\left\{x \in \mathbb{R}^{n}: l \leq x \leq u\right\},
$$

é a região factível, sendo $l \in(\mathbb{R} \cup\{-\infty\})^{n}, u \in(\mathbb{R} \cup\{+\infty\})^{n}$ e $[l]_{i}<[u]_{i}$.

A ideia original surgiu de aplicar o método de Newton não diferenciável (ver, por exemplo, Gaujoux (2005)) ao sistema de equações não diferenciáveis (1.22), obtido ao reformular as condições KKT (1.4) usando a fórmula introduzida por Fischer (1992).

$$
\left\{\begin{aligned}
\nabla q\left(x^{*}\right)-\sum_{i \in \mathcal{I}_{l}}\left[\lambda^{*}\right]_{i} e_{i}+\sum_{i \in \mathcal{I}_{u}}\left[\mu^{*}\right]_{i} e_{i}= & 0, \\
{[x-l+\lambda]_{i}-\sqrt{[x-l]_{i}^{2}+[\lambda]_{i}^{2}}=} & 0, \quad \forall i \in \mathcal{I}_{l}, \\
{[u-x+\mu]_{i}-\sqrt{[u-x]_{i}^{2}+[\mu]_{i}^{2}}=} & 0, \quad \forall i \in \mathcal{I}_{u} .
\end{aligned}\right.
$$

O "método" NQC usa o método do Gradiente Espectral Projetado, apresentado em Birgin et al. $(2000,2001)$ ), para definir em que face trabalhar e o método de Newton (ver, por exemplo, Nocedal e Wright (1999)), para calcular o minimizador da quadrática reduzida a esta face. 


\section{Algoritmo 1.3 NQC}

Dados um ponto inicial $x_{0}$ e $\epsilon>0$. Seja $s_{k}=x_{k}-x_{k-1}$ e $y_{k}=\nabla q\left(x_{k}\right)-$ $\nabla q\left(x_{k-1}\right), s_{0}=x_{0}+t s * g p, y_{0}=\nabla q\left(s_{0}\right), t s=10^{-8} \max \left\{\frac{\max _{i}\left\{\left|\left[x_{0}\right]_{i}\right|\right\}}{\max _{i}\left\{[g p]_{i}\right\}}, 1\right\} \mathrm{e}$ $[g p]_{i}=\min \left\{[u]_{i}, \max \left\{[l]_{i},\left[x_{0}-\nabla q\left(x_{0}\right)\right]_{i}\right\}\right\}-\left[x_{0}\right]_{i}$

Passo 1: Faça $k \leftarrow 0$.

Passo 2: Se $\left\|P_{\Omega_{\square}}\left(x_{k}-\nabla q\left(x_{k}\right)\right)-x_{k}\right\|<\epsilon$ então pare com $x_{k}$ como solução.

Passo 3: Calcule o tamanho de passo $\sigma$

$$
\sigma= \begin{cases}1, & \text { se } s_{k}^{T} y_{k} \leq 0, \\ \min \left\{10^{10}, \max \left\{10^{-10}, \frac{s_{k}^{T} s_{k}}{s_{k}^{T} y_{k}}\right\}\right\}, & \text { caso contrário. }\end{cases}
$$

Passo 4: Projete $x_{k}-\sigma \nabla q\left(x_{k}\right)$ no conjunto viável $\Omega_{\square}$, obtendo o ponto $\bar{x}_{k}$. Ou seja, faça $\bar{x}_{k}=P_{\Omega_{\square}}\left(x_{k}-\sigma \nabla q\left(x_{k}\right)\right)$.

Passo 5: Defina $\mathcal{A}_{l}$ o conjunto de índices para os quais $\left[\bar{x}_{k}\right]_{i}=[l]_{i}$ e $\mathcal{A}_{u} \mathrm{o}$ conjunto de índices para os quais $\left[\bar{x}_{k}\right]_{i}=[u]_{i}$.

Passo 6: Se os conjuntos $\mathcal{A}_{l}$ e $\mathcal{A}_{u}$ já foram visitados pelo algoritmo, pare declarando a existência de um ciclo.

Passo 7: Considere o problema

$$
\begin{array}{ll}
\text { Minimizar } q(x) & \\
\text { sujeita a } & {[x]_{i}=[l]_{i}, \quad i \in \mathcal{A}_{l},} \\
& {[x]_{i}=[u]_{i}, \quad i \in \mathcal{A}_{u},}
\end{array}
$$

e o resolva através do sistema de Newton.

Passo 8: Faça $k \leftarrow k+1$ e volte ao Passo 1.

Note que não é pedido nem decréscimo da função, nem viabilidade dos iterandos. Isso permite que o método seja eficiente, mas, em alguns casos, pode falhar por entrar em um ciclo.

\subsection{Resultados numéricos}

Nesta seção, apresentamos os resultados numéricos obtidos ao usar o método Moré-Toraldo e o "método" NQC para resolver problemas de programação quadrática com restrições de caixa ou irrestritos. 
Implementamos o método Moré-Toraldo em Fortran 77. O "método" NQC foi implementado pela orientadora, Profa. Dra. Marina Andretta, durante seu pós-doutorado, também em Fortran 77. Daqui em diante, chamaremos estas duas implementações de GPCG e NQC, respectivamente. Os códigos foram compilados em gfortran, usando o sistema operacional Ubuntu 11.10. Os experimentos numéricos foram realizados em um PC com Processador $2^{\text {a }}$ Geração Intel Core i3 2310M, 2.1 GHz, 4GB de memória.

Os resultados para NQC foram obtidos utilizando $\epsilon=10^{-5}$ no critério de convergência (Passo 2 do Algoritmo 1.3).

Os resultados para GPCG, foram obtidos com os seguintes parâmetros:

$$
\eta_{1}=0.1, \quad \eta_{2}=0.25, \quad \mu=0.1, \quad \epsilon=10^{-8}
$$

Lembrando que $\eta_{1}$ aparece no critério de parada do método dos Gradientes Conjugados (1.12), $\eta_{2}$ no critério de parada do método do Gradiente Projetado (1.16), $\mu$ na condição de decréscimo suficiente da busca projetada (1.17), e $\epsilon$ no Passo 7 do algoritmo GPCG.

Nestes resultados, para fazer uma comparação mais justa do método MoréToraldo com o "método" NQC, na implementação GPCG usamos o critério de parada

$$
\left\|\nabla_{\Omega_{\square}} q(x)\right\|_{2} \leq \tau
$$

$\operatorname{com} \tau=10^{-5}$

Usamos GPCG e NQC para resolver todos os problemas de programação quadrática com restrições de caixa e irrestritos da coleção CUTEr (Bongartz et al. (1995)), totalizando 51 problemas. Alguns destes problemas tem mais de uma dimensão possível e, nestes casos, resolvemos o problema usando as três dimensões maiores, totalizando 116 problemas. Os pontos iniciais usados são os sugeridos para cada problema da coleção CUTEr.

As Hessianas $H$ dos problemas tomados da coleção CUTEr podem não ser definidas positivas. Nos experimentos de ambos os métodos, em vez de resolver 
o problema original, resolvemos o problema

$$
\begin{array}{ll}
\text { Minimizar } & \frac{1}{2} x^{T}(H+\rho I) x+b^{T} x \\
\text { sujeita a } & x \in \Omega_{\square},
\end{array}
$$

com $\rho$ tal que $(H+\rho I)$ é definida positiva. Em NQC, para resolver os sistemas lineares Newtonianos, é usada a rotina MA57 (Duff (2004)) da biblioteca HSL.

Os problemas NCVXBQP1, NCVXBQP2 e NCVXBQP3 da coleção CUTEr não foram resolvidos, já que, para deixar suas Hessianas definidas positivas e ter, assim, problemas convexos, precisaríamos somar na diagonal um número muito grande, e teríamos problemas totalmente diferentes dos originais. Os problemas HARKERP2 de dimensões 5000 e 10000 também não foram resolvidos, já que as suas matrizes Hessianas são densas. Os resultados da aplicação de GPCG e NQC ao problema BQPGAUSS não foram levados em consideração, já que, ao aplicar GPCG, este parou por atingir o número máximo de iterações permitidas (5000). Ao aplicar NQC, este parou por encontrar um ciclo. Ao aplicar GPCG nos problemas ODNAMUR e PALMER1D, este parou sem satisfazer o critério de convergência. Para o problema ODNAMUR, parou por atingir o tempo máximo de execução permitido (1 hora) e para o problema PALMER1D, parou por progresso insuficiente. Ao aplicar NQC nestes dois problemas, o critério de convergência foi satisfeito. Nos 108 problemas restantes o critério de convergência foi satisfeito na aplicação dos algoritmos GPCG e NQC. Os três problemas apresentados nas Tabelas 1.1-1.7 são repetidos por completude.

Os resultados obtidos pela aplicação de GPCG e NQC para a resolução dos problemas da coleção CUTEr podem ser encontrados nas Tabelas 1.8-1.11 e 1.12-1.15, respectivamente. Nestas tabelas, Nome é o nome do problema; $n$ é o número de variáveis do problema; $\rho$ é o número somado na diagonal da Hessiana do problema (1.21); Tempo é o tempo de execução em segundos; $k$ é o número de iterações; $f\left(x^{*}\right)$ é o valor da função no ponto final $x^{*}$ obtido pelos métodos; $n_{f g}$ é o número de avaliações da função-gradiente; $n_{h}$ o número de produtos Hessiana-vetor; $n_{f}$ é o número de avaliações da função; $n_{g}$ é número de avaliações do gradiente; SN é o número de vezes que é resolvido um sistema de Newton; GC e GP são o número de iterações do Gradiente Conjugado e do Gradiente Projetado, respectivamente; gpc e gpd são a norma do gradiente 
projetado contínuo no ponto $x^{*}$ obtido por NQC e a norma do gradiente projetado descontínuo no ponto $x^{*}$ obtido por GPCG, respectivamente; e ciclos é o tamanho do ciclo (zero se nenhum ciclo é encontrado).

Nas Tabelas 1.8-1.11, inf é igual a zero, se o critério de parada (1.23) é satisfeito; igual a 1, se o número máximo de iterações é atingido (definido como 5000); igual a 2 se progresso insuficiente; igual a -1 se o tempo máximo de execução é atingindo (definido como 3600 segundos). Nas Tabelas 1.12-1.15, inf é igual a zero, se o gradiente projetado contínuo é menor ou igual a $\epsilon$; igual a 4 se um ciclo é encontrado.

A fim de comparar os resultados obtidos ao aplicar GPCG e NQC, dizemos que dois valores de função $f_{1}$ e $f_{2}$ são iguais se

$$
\left|f_{1}-f_{2}\right| \leq \max \left\{10^{-10}, 10^{-6} \min \left\{\left|f_{1}\right|,\left|f_{2}\right|\right\}\right\} .
$$

Em 10 problemas o valor da função ao aplicar o NQC foi menor. GPCG produziu melhores soluções em 5 problemas. Nos 95 problemas restantes, ao aplicar ambos os métodos, os valores das funções foram iguais.

Estamos interessados em comparar, basicamente, o tempo gasto por cada método e a qualidade da solução encontrada por cada método. Para visualizar melhor esta comparação, construímos os gráficos do perfil de desempenho, que podem ser vistos nas Figuras 1.2-1.3. Nestes gráficos, usando o tempo como medida de desempenho, se um ponto da curva correspondente a um dos métodos vale $I$ no eixo das abscissas e $J$ no eixo das ordenadas, significa que este método resolve $(J \times 100) \%$ dos problemas em um tempo menor ou igual a $I$ vezes o tempo mais rápido. Os pontos mais significativos do gráfico são o mais à esquerda e o mais à direita. À esquerda medimos a eficiência dos métodos (verificamos a porcentagem de problemas para os quais cada método é o mais rápido) e à direita medimos a robustez (verificamos a porcentagem de problemas que cada método foi capaz de resolver).

Para a construção dos gráficos das Figuras 1.2 e 1.3, comparamos o tempo de execução de cada método. Para os gráficos da Figura 1.2, quando um dos dois métodos encontra valor de função maior para um mesmo problema, consideramos que este método levou tempo infinito para encontrar a solução. Para 
os gráficos da Figura 1.3, consideramos apenas os problemas para os quais ambos os métodos chegaram ao mesmo valor de função.

Observando os dados das Tabelas 1.8-1.15 e os gráficos das Figuras 1.2 e 1.3, podemos notar que NQC se mostrou um pouco mais robusto que GPCG (Robustez - GPCG 99,09\%, NQC 100\%). Por outro lado, GPCG se mostrou um pouco mais eficiente, tanto quando penalizamos os métodos que encontraram pontos com valores de função piores (Eficiência - GPCG 80,00\%, NQC $33,63 \%)$, como quando usamos na comparação apenas os problemas em que os valores de função são iguais (Eficiência - GPCG 80,73\%, NQC 33,02\%). 

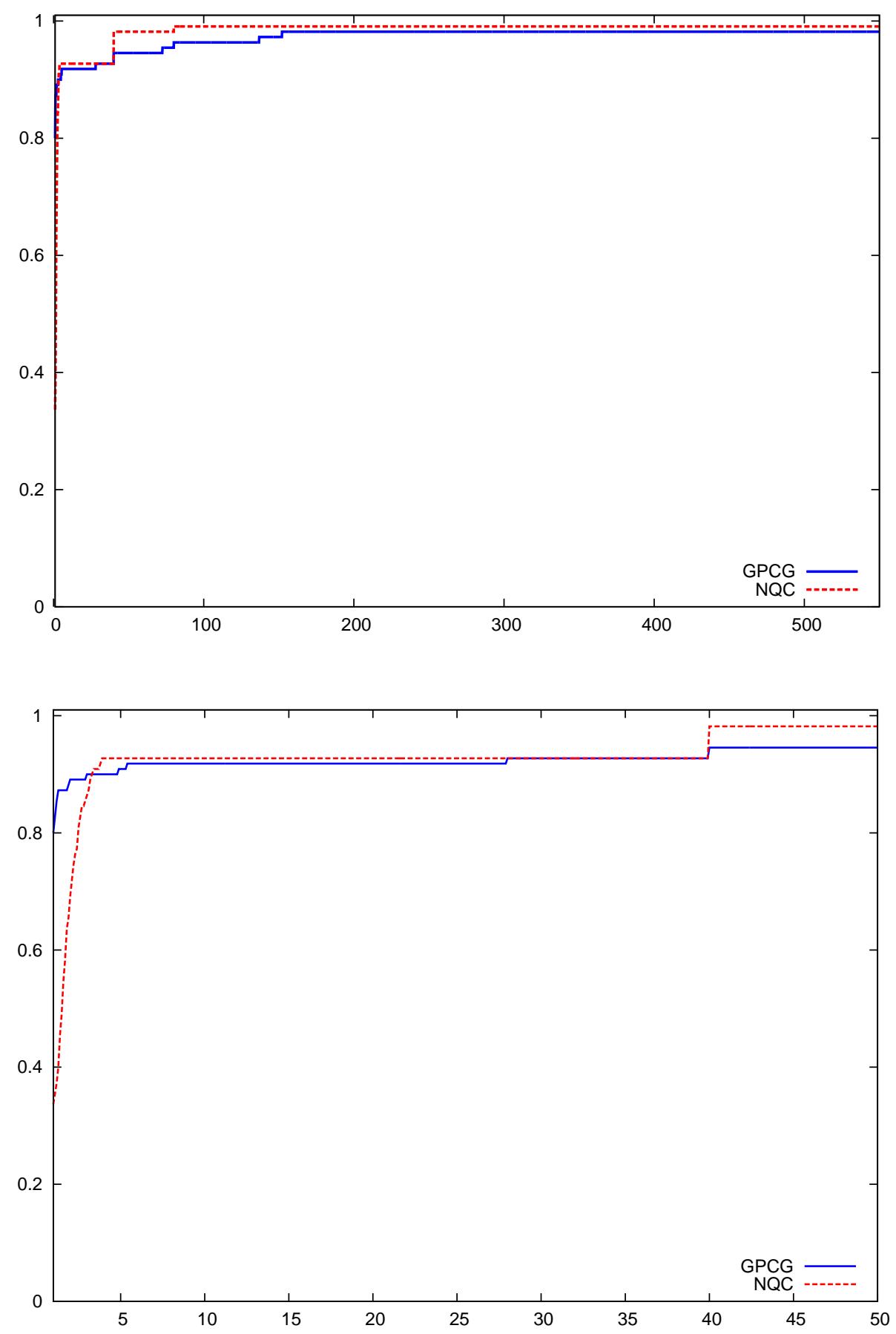

Figura 1.2: Curva de perfil de desempenho comparando GPCG com NQC usando tempo como medida de desempenho. O segundo gráfico é apenas um destaque da região mais à esquerda do primeiro gráfico. Para a construção destes gráficos, quando um dos dois métodos encontra valor de função diferente para um mesmo problema, consideramos que este método levou tempo infinito para encontrar a solução. 

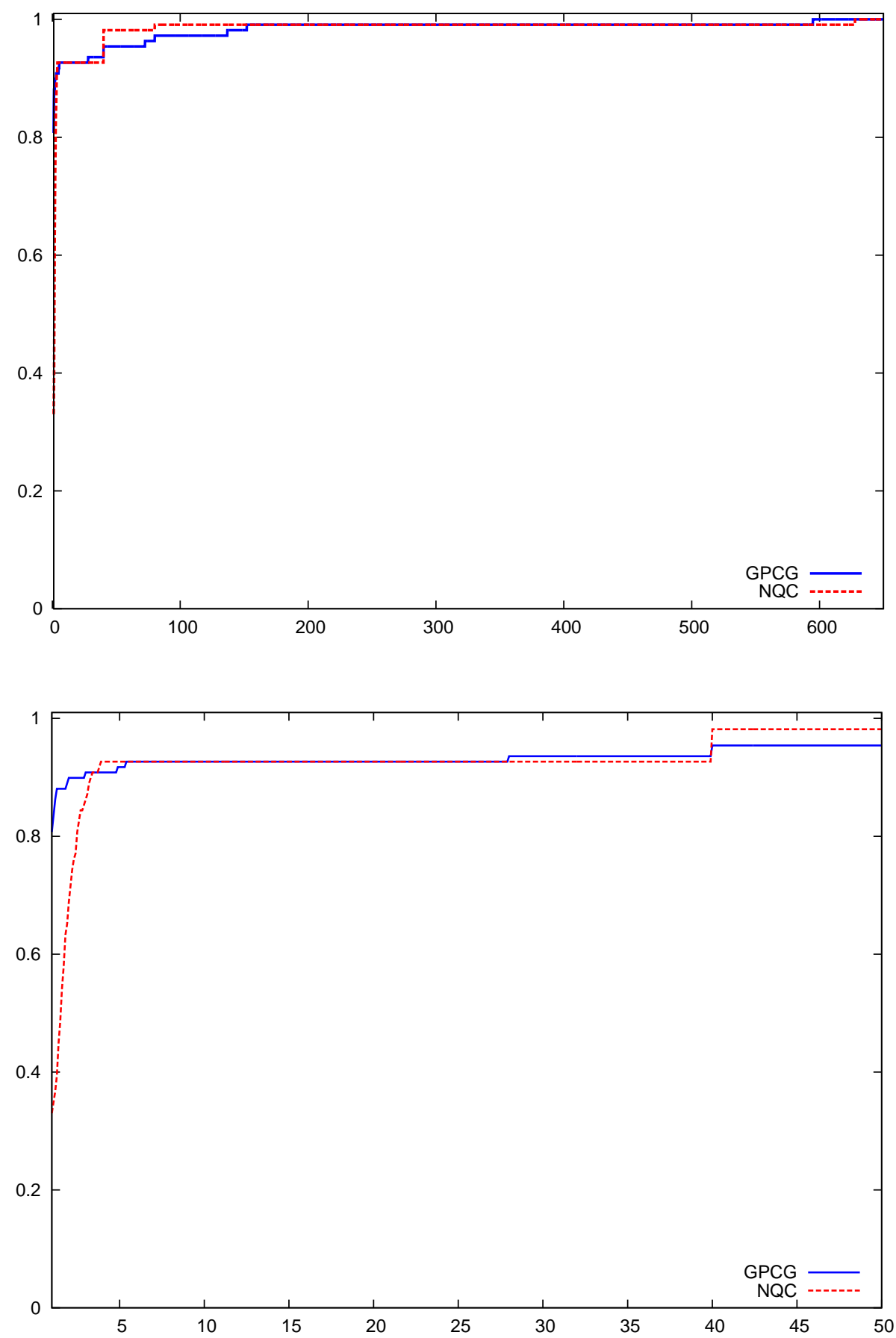

Figura 1.3: Curva de perfil de desempenho comparando GPCG com NQC usando tempo como medida de desempenho. O segundo gráfico é apenas um destaque da região mais à esquerda do primeiro gráfico. Para a construção destes gráficos, consideramos apenas os problemas para os quais ambos os métodos chegaram ao mesmo valor de função. 
三 *

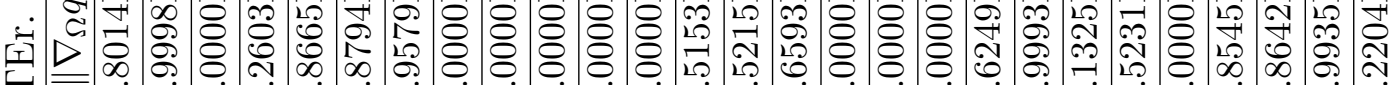

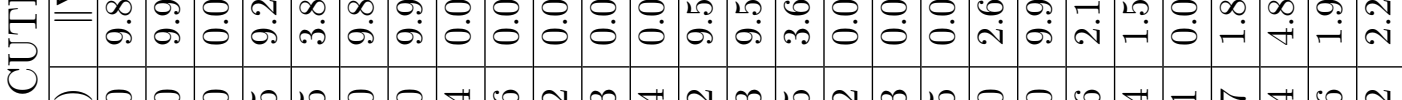
○ 仓

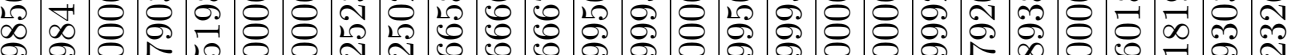
皃

$\frac{0}{0}$

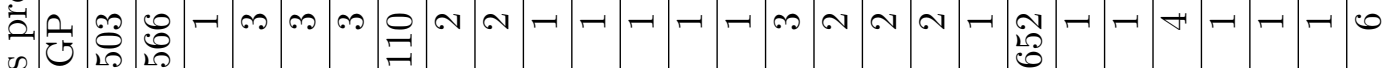
잉 


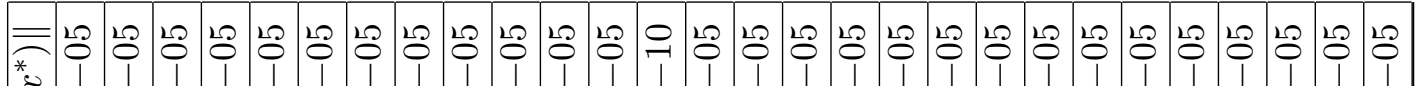

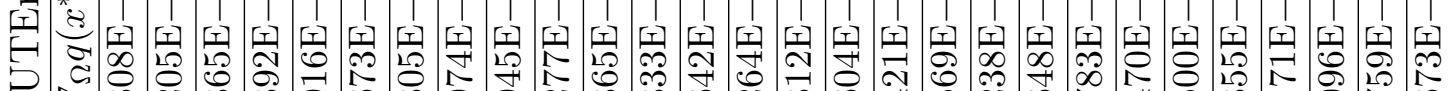

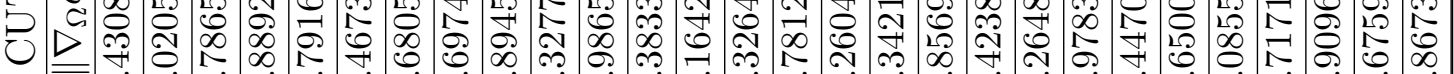

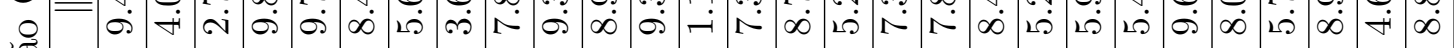

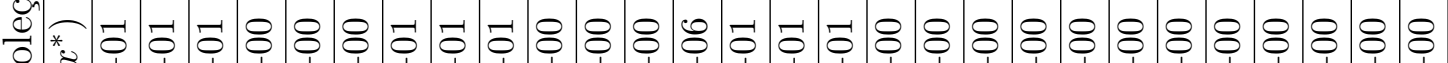
至 U द्वे 党

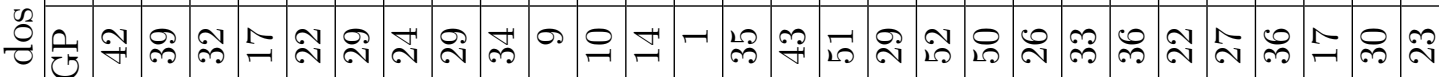
,

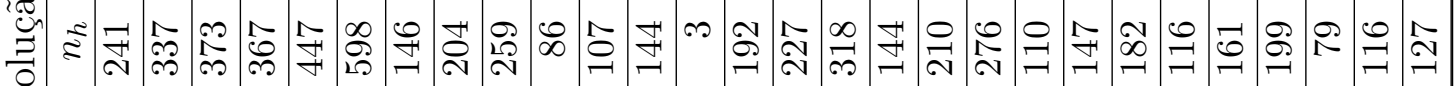

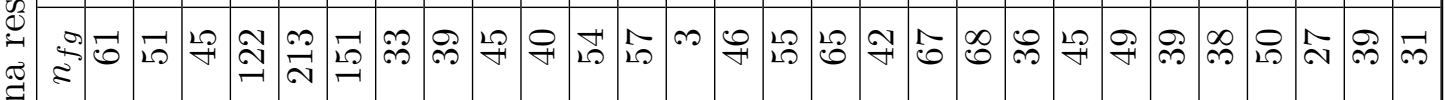

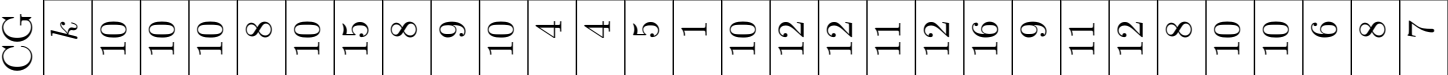
৩  ,

đ:

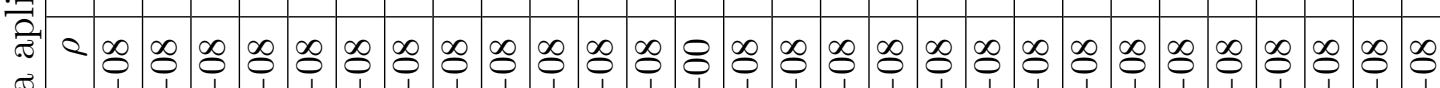

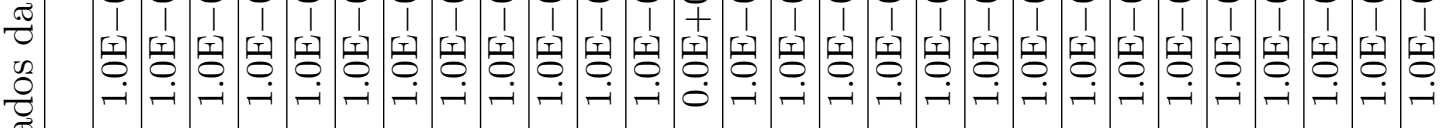

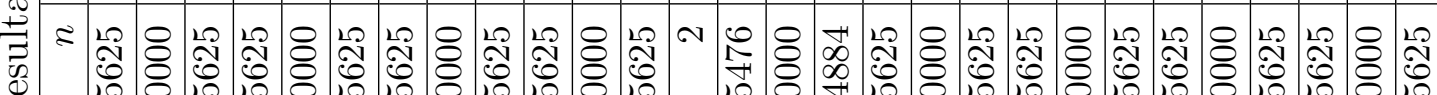

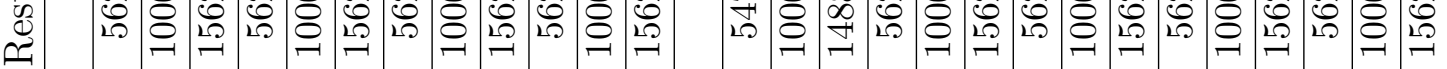
$\ddot{-}$

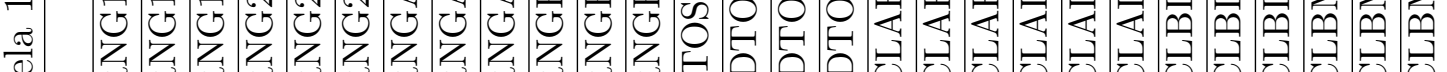
呈

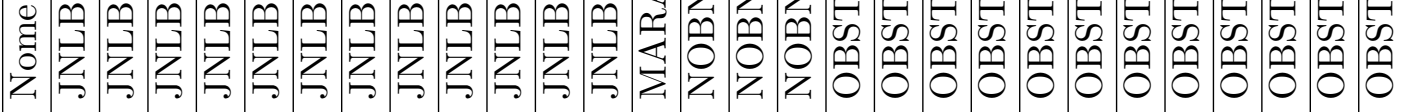




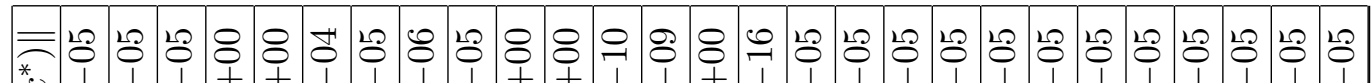

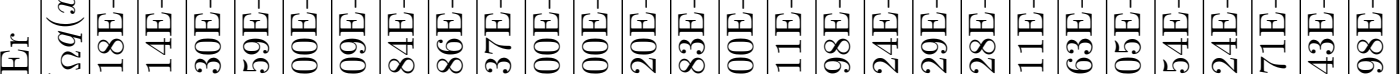

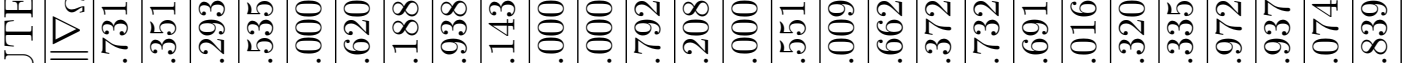

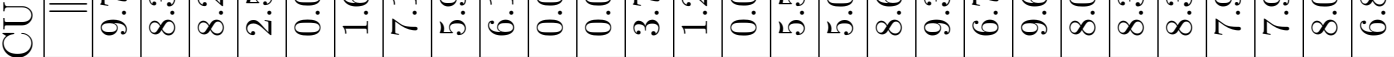
赵 兽

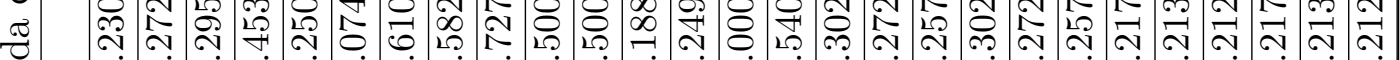
है

?

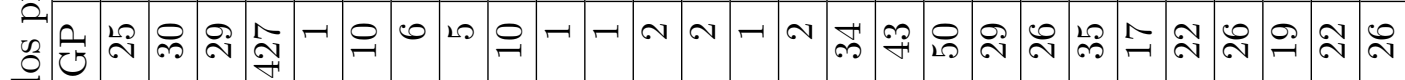

(a)

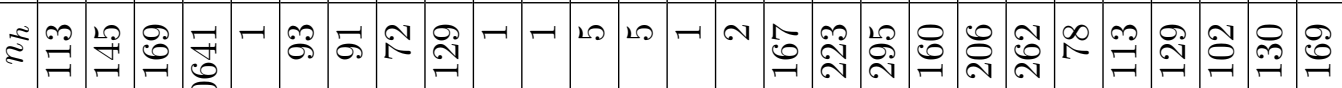

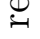

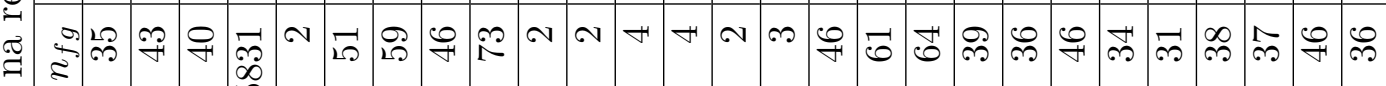

¿

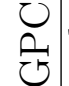

\& $\sigma=0$ 응

$\stackrel{0}{0}$

\&

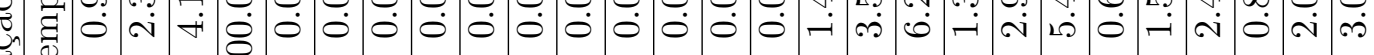

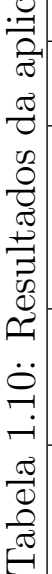

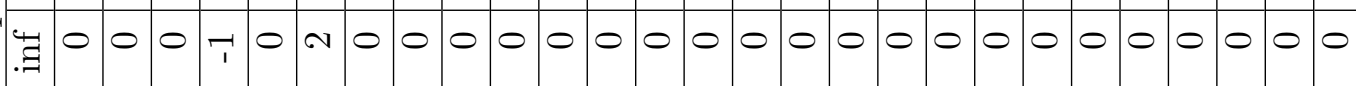

人

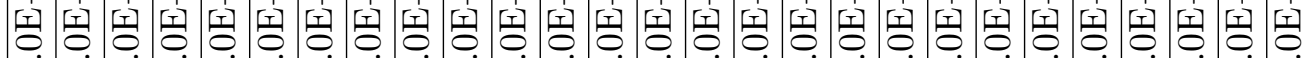

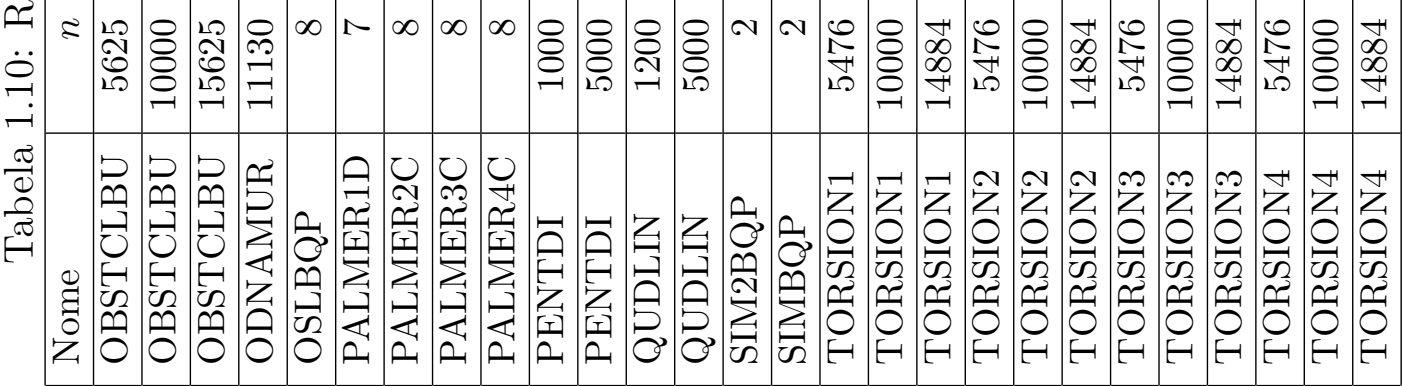




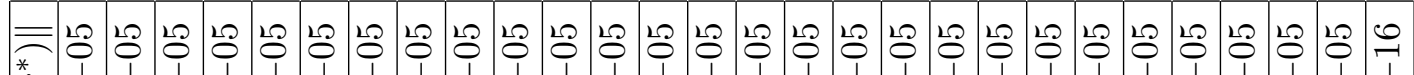
帒

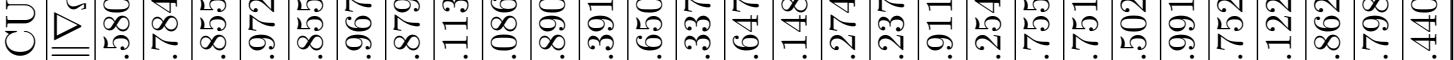

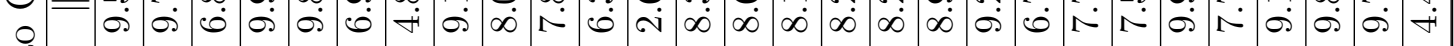

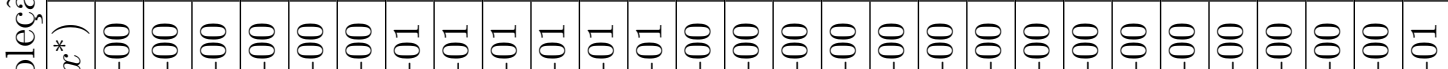

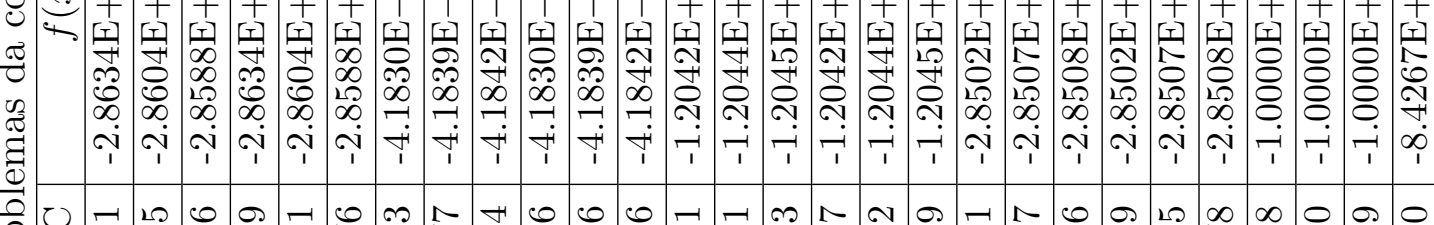
完

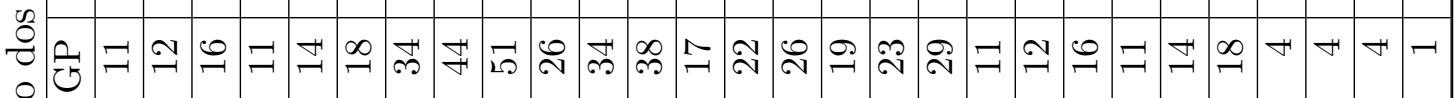
i。্তে

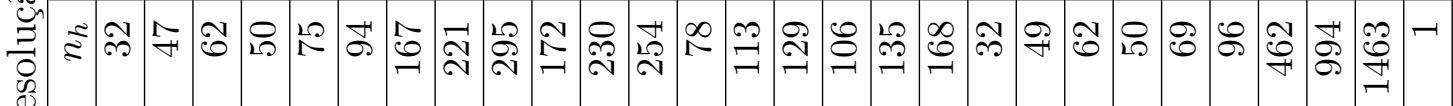

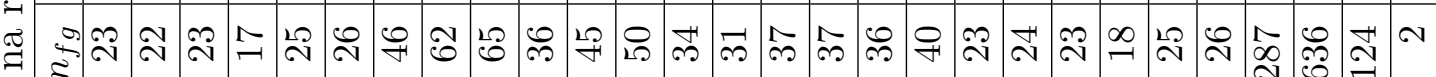
$\circlearrowright$

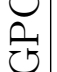

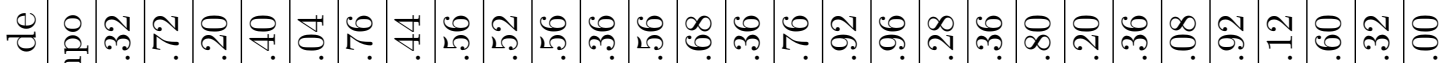
ı

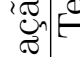

(1)

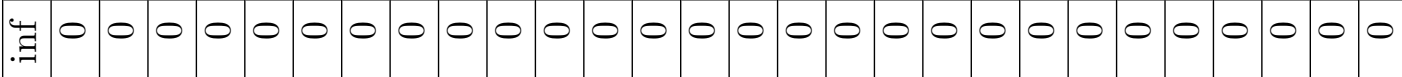

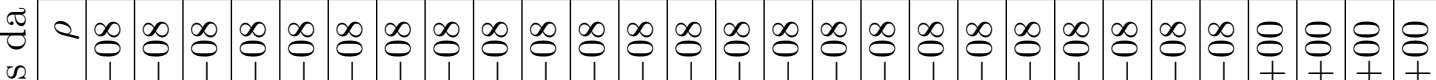

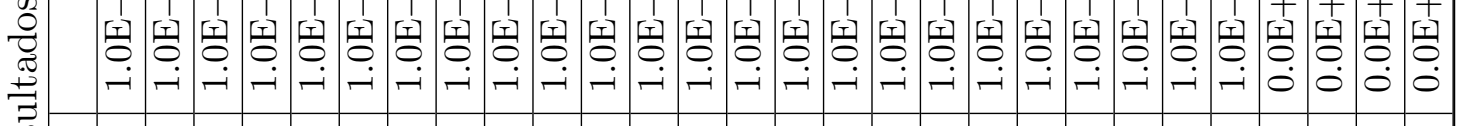

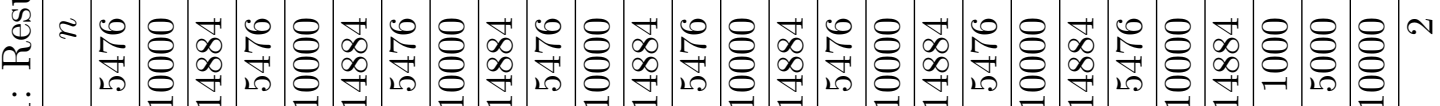

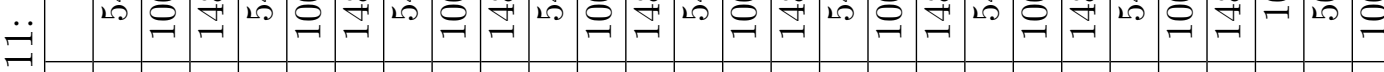

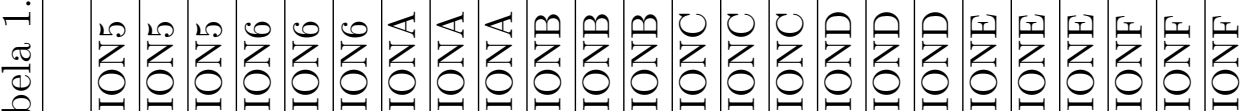

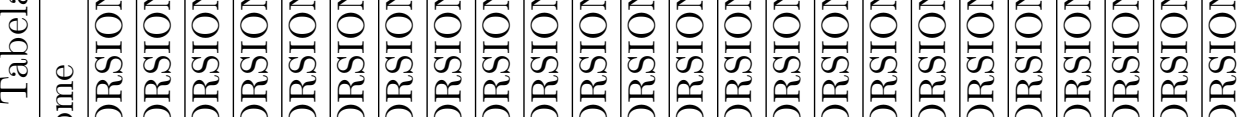
( 


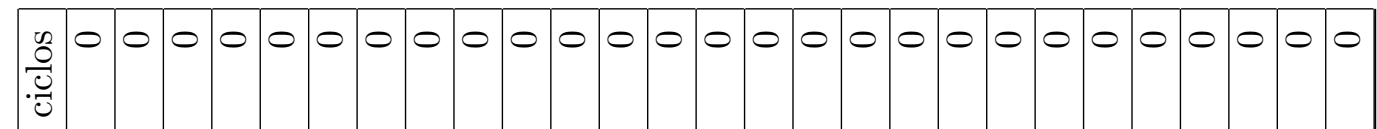

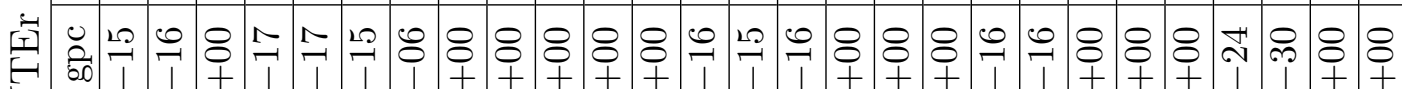

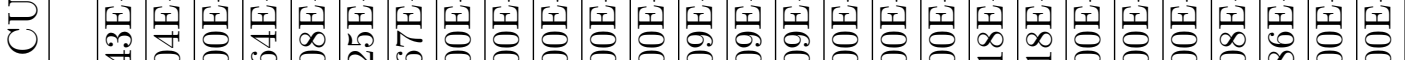

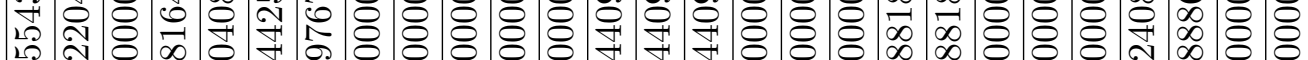


悉

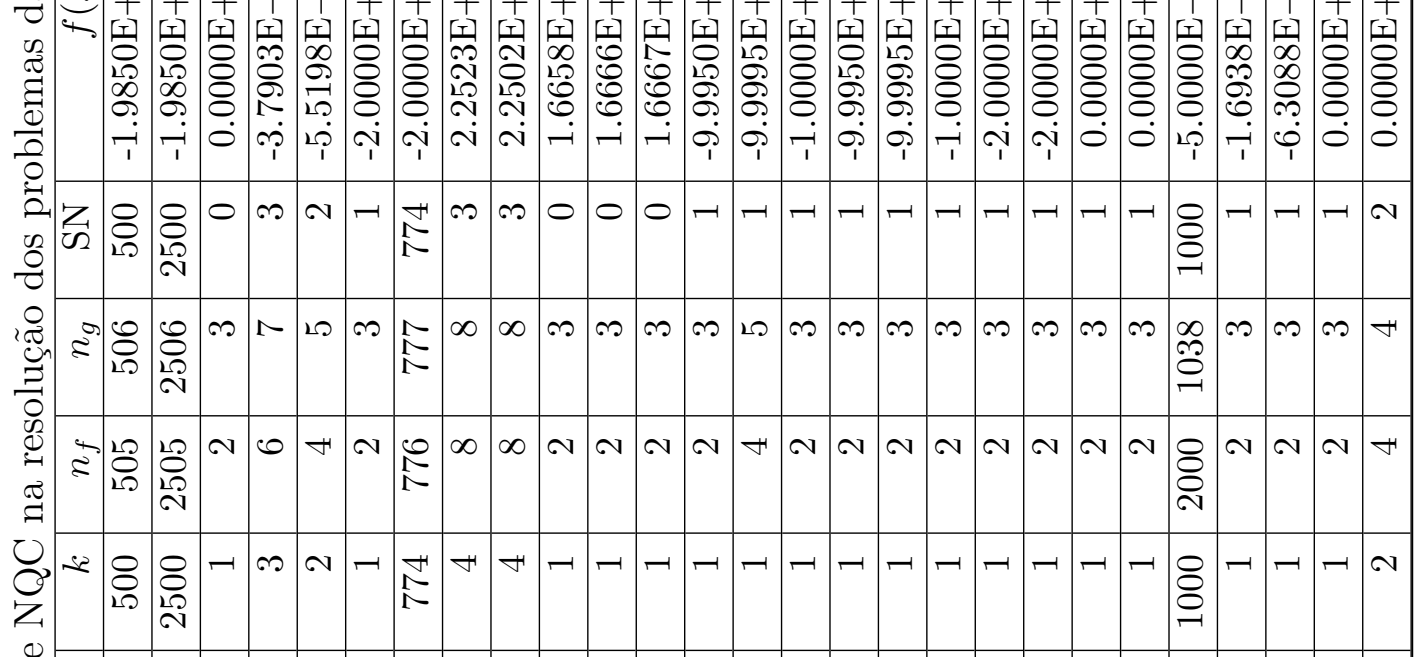

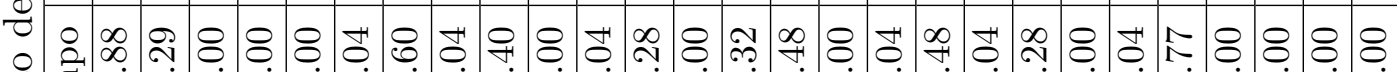
承

के

\%

○

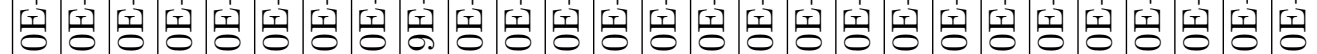

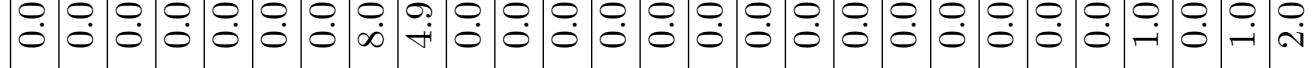
거

至

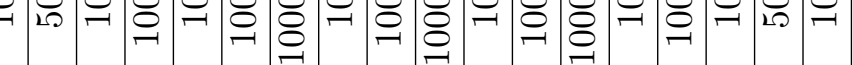
勿焉 \& 号商 
\begin{tabular}{|l|l|l|l|l|l|l|l|l|l|l|l|l|l|l|l|l|l|l|l|l|l|l|l|l|l|l|l|l|}
\hline 兵总 & 0 & 0 & 0 & 0 & 0 & 0 & 0 & 0 & 0 & 0 & 0 & 0 & 0 & 0 & 0 & 0 & 0 & 0 & 0 & 0 & 0 & 0 & 0 & 0 & 0 & 0 & 0 & 0
\end{tabular}

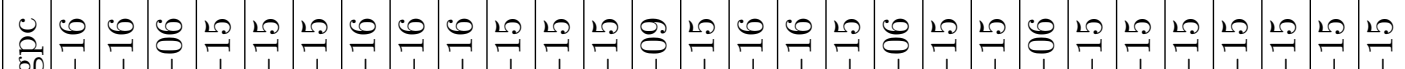

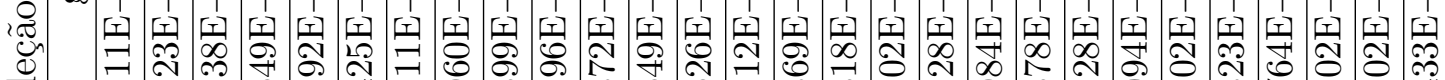

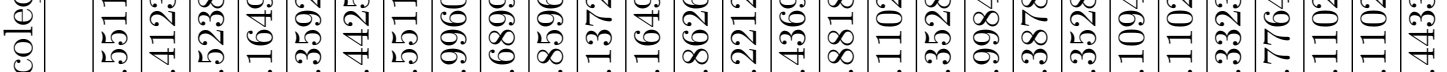

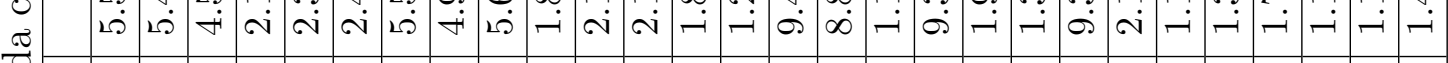
孔

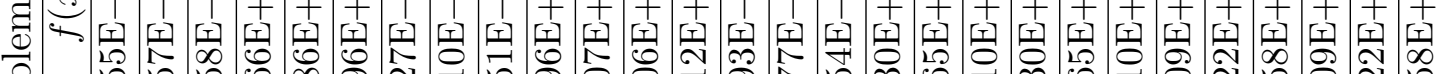
$\frac{10}{0}$

పี

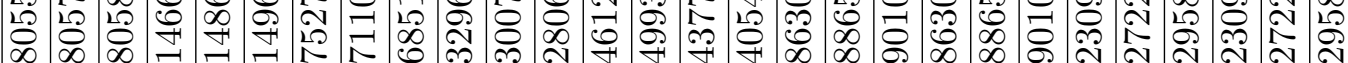
$\infty=-1 N$ N 0 m m ?용 烍

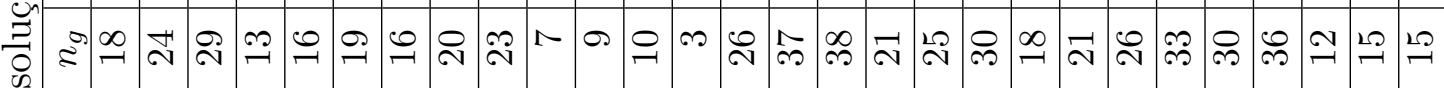

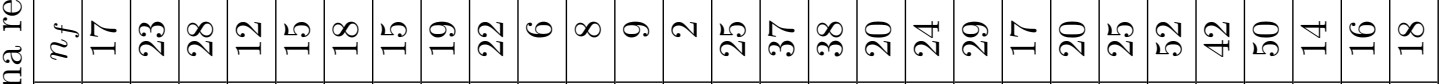

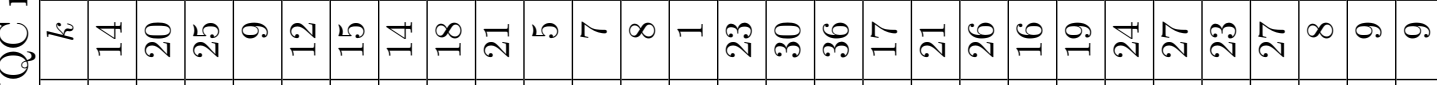

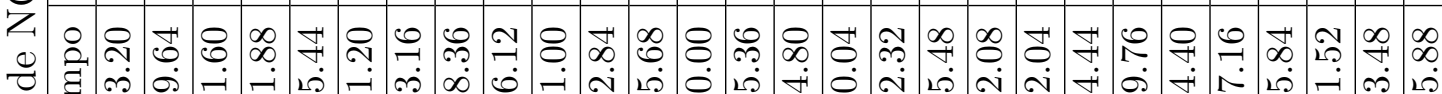
O :

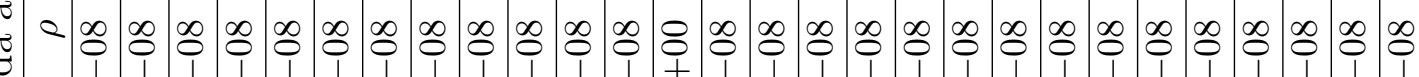

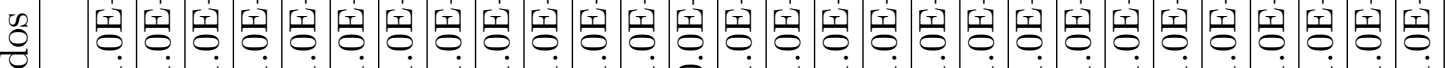

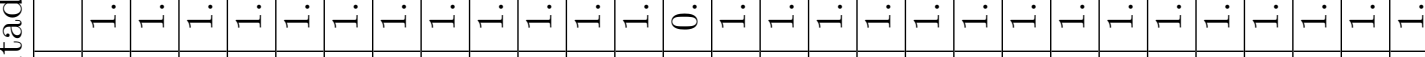
に

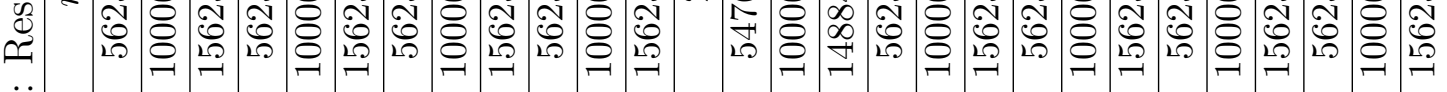

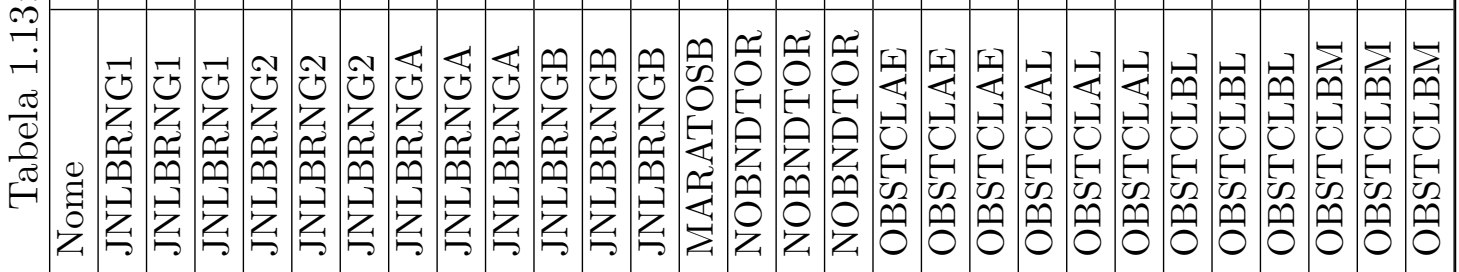




\begin{tabular}{|c|c|c|c|c|c|c|c|c|c|c|c|c|c|c|c|c|c|c|c|c|c|c|c|c|c|c|c|}
\hline & $\infty$ & & & & 0 & |c & 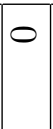 & & & & & & & & 0 & & & & & & & & & & & & \\
\hline & 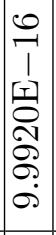 & 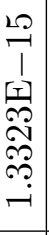 & 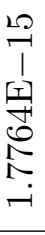 & 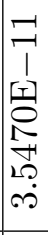 & 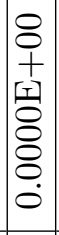 & 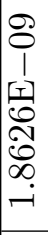 & 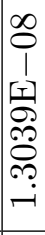 & $\begin{array}{r}8 \\
0 \\
1 \\
1 \\
0 \\
0 \\
0 \\
0 \\
\\
+ \\
\end{array}$ & 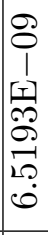 & \begin{tabular}{|l}
1 \\
$\mid 1$ \\
$\infty$ \\
$\infty$ \\
$\infty$ \\
$\infty$ \\
$\infty$ \\
$\infty$ \\
$\infty$ \\
\end{tabular} & \begin{tabular}{l}
1 \\
\multicolumn{1}{c}{} \\
$\infty$ \\
$\infty$ \\
$\infty$ \\
$\infty$ \\
$\infty$ \\
\end{tabular} & 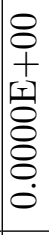 & $\begin{array}{l}+ \\
\text { 帘 } \\
8 \\
8 \\
8 \\
0 \\
0\end{array}$ & $\begin{array}{l}1 \\
8 \\
8 \\
8 \\
8\end{array}$ & $\begin{array}{l}+ \\
\text { 国 } \\
8 \\
8 \\
0 \\
0 \\
0\end{array}$ & 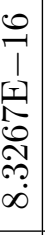 & & 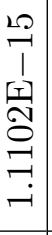 & $\begin{array}{l}1 \\
\text { II } \\
\infty \\
\infty \\
\infty \\
\infty \\
\infty \\
\infty\end{array}$ & & 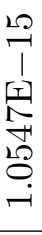 & $\begin{array}{c}0 \\
-1 \\
1 \\
1 \\
1 \\
0 \\
\infty \\
\infty \\
\infty \\
\infty \\
\infty\end{array}$ & $\overbrace{\substack{1 \\
\text { II }}}^{10}$ & 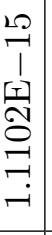 & 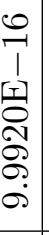 & & 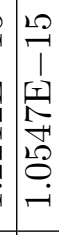 \\
\hline & 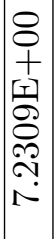 & 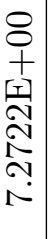 & 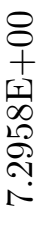 & 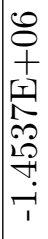 & 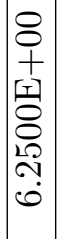 & 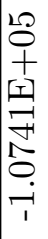 & 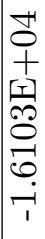 & 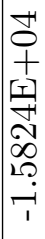 & 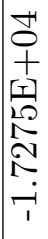 & $\begin{array}{l}-1 \\
0 \\
1 \\
1 \\
1 \\
8 \\
8 \\
0 \\
10 \\
i \\
1\end{array}$ & $\begin{array}{l}1 \\
\text { T1 } \\
8 \\
0 \\
0 \\
10 \\
1 \\
1\end{array}$ & 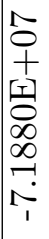 & 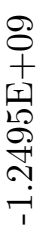 & 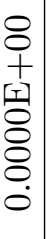 & $\begin{array}{c}8 \\
8 \\
+ \\
\text { 国 } \\
8 \\
8 \\
8 \\
0 \\
0\end{array}$ & Pُ & $\stackrel{\substack{* \\
\hdashline}}{ }$ & 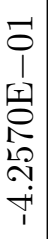 & 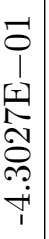 & 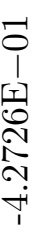 & $\stackrel{N}{\sim}$ & 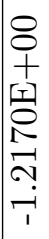 & 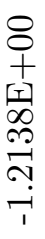 & 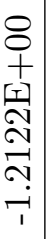 & 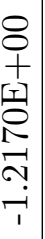 & & $\left\{\begin{array}{l}\sigma \\
\sigma \\
\sigma \\
\sigma\end{array}\right.$ \\
\hline 㮩 & $\stackrel{0}{-1}$ & $\stackrel{\curvearrowright}{\curvearrowright}$ & $\stackrel{N}{N}$ & $\stackrel{-1}{\prime}$ & -1 & -1 & - & - & -1 & -1 & -1 & N & - & 0 & $\neg$ & $\ddot{\sim}$ & $\bar{\omega}$ & $\hat{\infty}$ & $\stackrel{\infty}{\rightarrow}$ & $\stackrel{0}{0}$ & $\sigma$ & $\exists$ & $\stackrel{10}{-1}$ & $\stackrel{\theta}{-}$ & +1 & & \\
\hline$\stackrel{0}{0}$ & $\stackrel{\Im}{\sim}$ & $\underset{\sim}{\curvearrowright}$ & $\stackrel{\leftrightarrow}{\sim}$ & $\stackrel{\infty}{-}$ & & $\infty$ & p & $\infty$ & $\infty$ & $\infty$ & $\infty$ & $\nabla$ & $\infty$ & 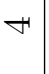 & 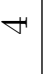 & $\stackrel{\circ}{\circ}$ & m & S) & $\stackrel{\simeq}{\simeq}$ & $\vec{N}$ & & $\mathscr{9}$ & $\stackrel{\sim}{\sim}$ & $\vec{\nabla}$ & $\stackrel{\infty}{-}$ & & \\
\hline$\stackrel{u}{2}$ & 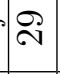 & $\vec{m}$ & 的 & $\stackrel{\sim}{\curvearrowright}$ & $\infty$ & $\sim$ & $N$ & $N$ & $N$ & $N$ & $N$ & $\infty$ & $N$ & $\infty$ & $\infty$ & $\stackrel{\leftrightarrow}{\sim}$ & ๙ิ & $\begin{array}{l}\infty \\
\infty \\
\infty\end{array}$ & $\stackrel{0}{-1}$ & $\stackrel{\curvearrowright}{\curvearrowright}$ & & $\stackrel{\mathcal{F}}{-}$ & $\stackrel{0}{-}$ & $\stackrel{\curvearrowright}{\circ}$ & $\stackrel{\sim}{\sim}$ & $\vec{N}$ & \\
\hline & $\underset{-1}{0}$ & ○ & & $\stackrel{\ominus}{-1}$ & -1 & -1 & -1 & -1 & -1 & -1 & -1 & N & - & $\neg$ & -1 & & $\vec{\sigma}$ & $\Lambda$ & $\stackrel{\oplus}{\rightarrow}$ & $\stackrel{0}{0}$ & & $\exists$ & $\stackrel{20}{\longrightarrow}$ & $\stackrel{g}{-}$ & $\exists$ & $\stackrel{\infty}{\sim}$ & \\
\hline 寓 & $\mid \begin{array}{c}\vec{H} \\
\stackrel{\sim}{\sim}\end{array}$ & $\bar{\delta}$ & \begin{tabular}{l}
$\infty$ \\
$\infty$ \\
$\infty$ \\
\hdashline
\end{tabular} & 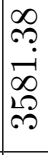 & 8 & $\stackrel{8}{0}$ & $\stackrel{b}{0}_{0}$ & $\stackrel{8}{\stackrel{8}{0}}$ & $\stackrel{8}{8}$ & \& & & $\stackrel{8}{8}$ & $\begin{array}{l}8 \\
0 \\
0\end{array}$ & 8 & \begin{tabular}{l}
8 \\
\hdashline \\
0
\end{tabular} & & & 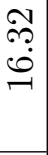 & 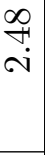 & קִ & & $\begin{array}{l}\stackrel{0}{1} \\
\stackrel{0}{0}\end{array}$ & $\begin{array}{l}\stackrel{8}{0} \\
\text { ì }\end{array}$ & $\begin{array}{c}\infty \\
\stackrel{\infty}{+} \\
\dot{+}\end{array}$ & & & \\
\hline & & & 0 & & & 0 & lo & & 0 & 0 & & 0 & 0 & 0 & 0 & 0 & 0 & 0 & 0 & 0 & & 0 & 0 & 0 & 0 & 0 & \\
\hline & $\mid \begin{array}{c}\infty \\
0 \\
1 \\
1 \\
0 \\
0 \\
-1\end{array}$ & $\begin{array}{c}0 \\
0 \\
1 \\
1 \\
\text { İ } \\
0 \\
-i\end{array}$ & $\begin{array}{l}\infty \\
0 \\
1 \\
1 \\
\text { - } \\
0 \\
\\
- \\
-\end{array}$ & $\begin{array}{l}8 \\
0 \\
+ \\
\text { I } \\
\text { O } \\
\dot{0}\end{array}$ & $\begin{array}{l}8 \\
0 \\
+ \\
\text { 团 } \\
0 \\
\dot{0}\end{array}$ & $\begin{array}{l}8 \\
0 \\
+ \\
\text { 国 } \\
0 \\
0\end{array}$ & $\mid \begin{array}{l}8 \\
+ \\
+ \\
\text { 团 } \\
0 \\
0 \\
0\end{array}$ & $\begin{array}{l}8 \\
0 \\
+ \\
1 \\
0 \\
0 \\
0\end{array}$ & $\mid \begin{array}{l}8 \\
+ \\
+ \\
\text { I } \\
0 \\
0 \\
0\end{array}$ & $\begin{array}{l}8 \\
0 \\
+ \\
\text { 至 } \\
0 \\
0\end{array}$ & $\begin{array}{l}8 \\
0 \\
+ \\
\text { 禾 } \\
0 \\
0\end{array}$ & 8 & $\begin{array}{l}8 \\
\stackrel{8}{+} \\
+ \\
\text { I } \\
0 \\
\dot{v}\end{array}$ & $\begin{array}{l}8 \\
0 \\
+ \\
\text { I } \\
0 \\
0 \\
0\end{array}$ & $\begin{array}{l}8 \\
8 \\
+ \\
\text { 至 } \\
0 \\
\dot{0}\end{array}$ & & & & $\begin{array}{l}\infty \\
0 \\
1 \\
1 \\
\text { II } \\
0 \\
- \\
-\end{array}$ & & & $\begin{array}{l}\infty \\
0 \\
1 \\
1 \\
1 \\
0 \\
0 \\
-1\end{array}$ & $\begin{array}{l}\infty \\
0 \\
1 \\
1 \\
\text { 1 } \\
0 \\
\\
-\end{array}$ & - & $\begin{array}{c}\infty \\
0 \\
0 \\
1 \\
0 \\
0 \\
0 \\
-i\end{array}$ & & 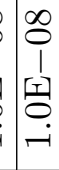 \\
\hline & $\begin{array}{l}20 \\
\stackrel{2}{0} \\
20 \\
20\end{array}$ & 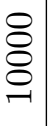 & & & $\infty$ & N & $\infty$ & $\infty$ & $\infty$ & 8 & & & & $\sim$ & 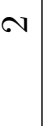 & & & & & & & 0 & $\S$ & -1 & $\begin{array}{l}0 \\
\stackrel{2}{2}\end{array}$ & & 4 \\
\hline & 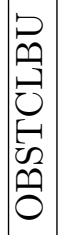 & 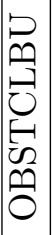 & $\begin{array}{l}0 \\
\qquad \\
\text { ins }\end{array}$ & 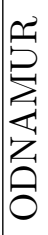 & 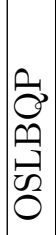 & 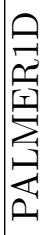 & 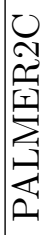 & 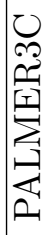 & & & f & & & $\stackrel{\Xi}{\Im}$ & $F$ & & & $\frac{\overrightarrow{2}}{\sqrt{2}}$ & $=$ & & & 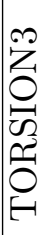 & 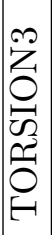 & 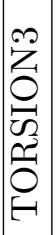 & 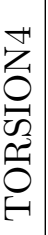 & $\underline{\tilde{N}}$ & 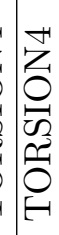 \\
\hline
\end{tabular}




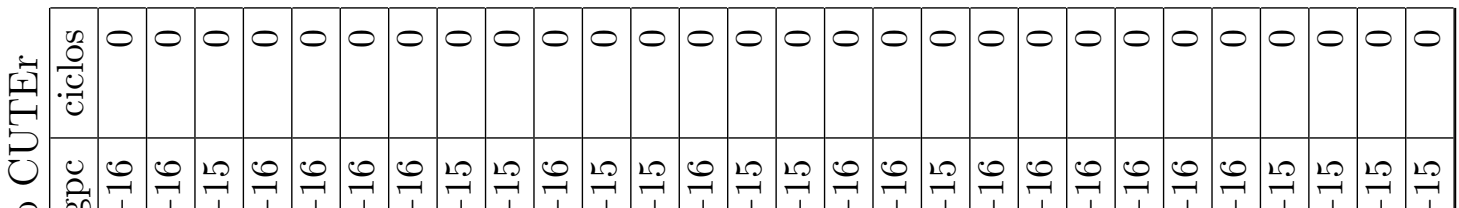

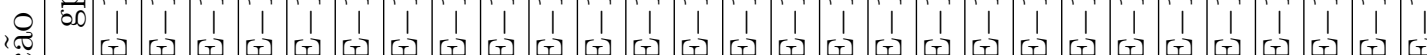

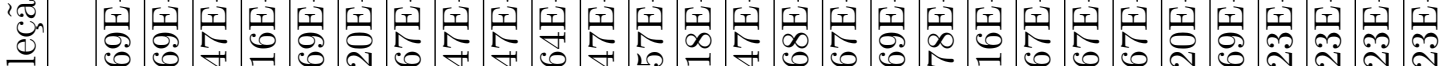
范

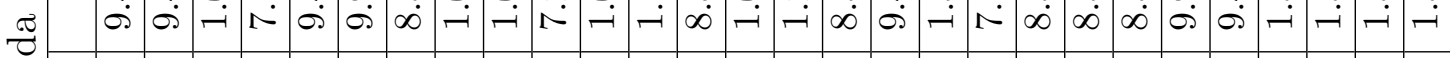
‡

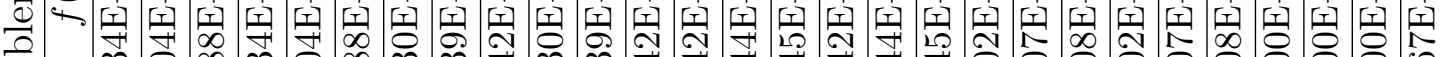

응

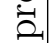

$\stackrel{2}{0}$

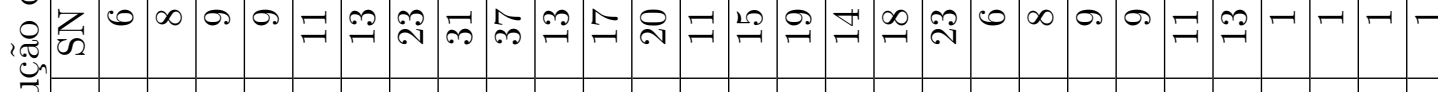

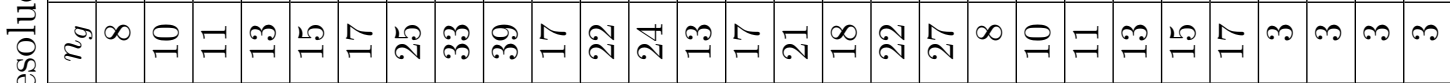

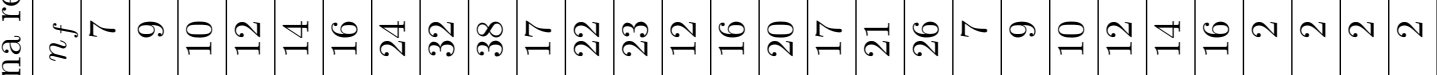

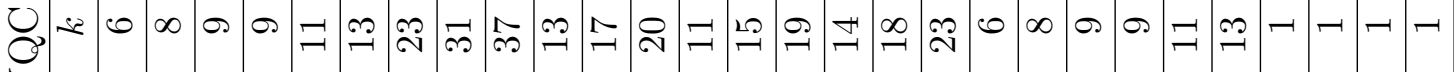
에 ت

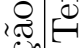

.

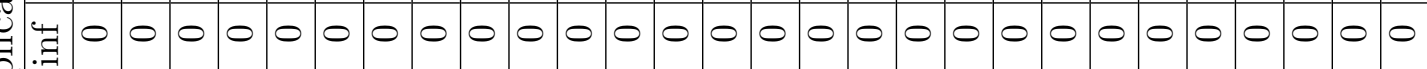

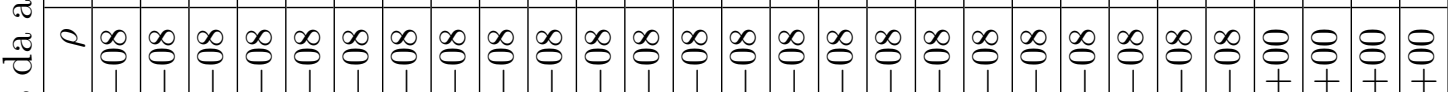

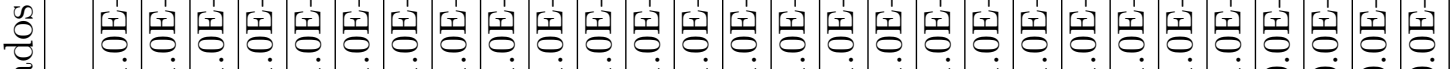
욤

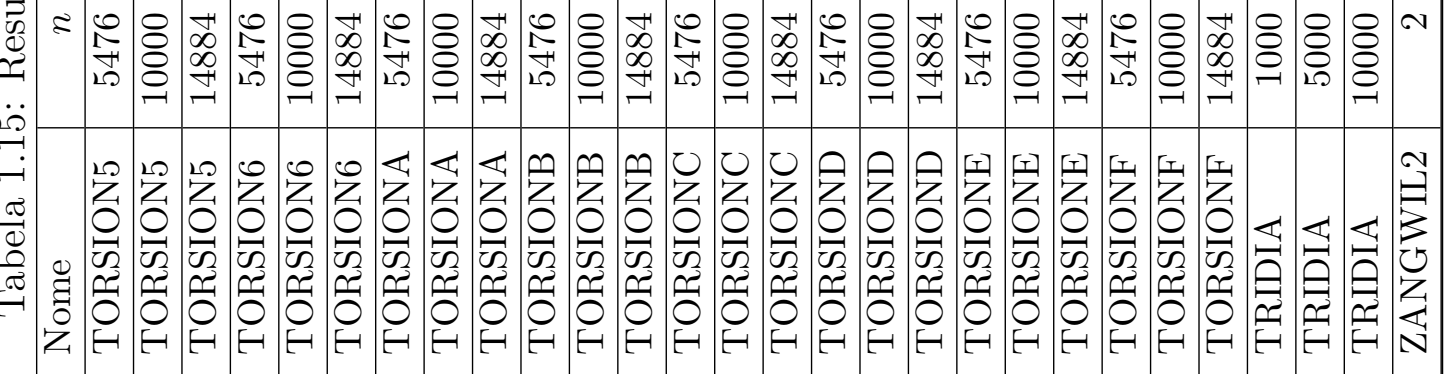


Capítulo

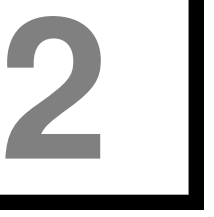

\section{Método para resolução de problemas de minimização com restrições lineares}

Neste capítulo, descrevemos em detalhes GENLIN, um método eficiente para resolução do problema de minimização com restrições lineares, apresentado em Andretta et al. (2010).

O problema de minimização com restrições lineares a ser resolvido por GENLIN é

$$
\begin{array}{ll}
\text { Minimizar } & f(x) \\
\text { sujeita a } & x \in \Omega=\left\{x \in \mathbb{R}^{n} \mid A x=b, C x \leq d, l \leq x \leq u\right\}
\end{array}
$$

onde $f$ é uma função em $\mathcal{C}^{2}, x \in \mathbb{R}^{n}, A \in \mathbb{R}^{r \times n}, b \in \mathbb{R}^{r}, C \in \mathbb{R}^{m \times n}, d \in \mathbb{R}^{m}, l \in$ $(\mathbb{R} \cup\{-\infty\})^{n}, u \in(\mathbb{R} \cup\{+\infty\})^{n}$ e $[l]_{i}<[u]_{i}, 1 \leq i \leq n$.

GENLIN é um método de restrições ativas e sua implementação faz parte do Projeto TANGO (Trustable Algorithms for Nonlinear General Optimization). A seguir, definimos formalmente o que é face do conjunto factível $\Omega$, conceito muito usado no estudo de métodos de restrições ativas.

Definição 2.1. Para todo $I \subset\{1,2, \ldots, m\}$ e $J \subset\{1,2, \ldots, 2 n\}$ definimos uma 
face do conjunto factivel $\Omega$ por

$$
\begin{aligned}
F_{I, J}=\left\{x \in \Omega \mid \quad a^{k} x\right. & =[b]_{k}, 1 \leq k \leq r, \\
c^{i} x & =[d]_{i}, \text { se } i \in I, \\
c^{i} x & <[d]_{i}, \text { se } i \notin I, \\
{[x]_{j} } & =[l]_{j}, \text { se } j \in J, \\
& {[x]_{j}=[u]_{j}, \text { se } n+j \in J, } \\
& {\left.[l]_{j}<[x]_{j}<[u]_{j}, \text { se } j \notin J, n+j \notin J\right\}, }
\end{aligned}
$$

onde $a^{k}$ é a k-ésima linha da matriz $A$ e $c^{i}$ é a i-ésima linha da matriz $C$.

O poliedro $\Omega$ é a união de todas as faces. As restrições lineares de igualdade, as restrições $i$ tais que $i \in I$ e $j$ tais que $j \in J$ são chamadas de restrições ativas. Definimos $V_{I, J}$ o menor espaço afim que contém $F_{I, J}$ e $S_{I, J}$ o subespaço linear paralelo a $V_{I, J}$.

De maneira análoga à que é feita no Capítulo 1, a seguir definimos o gradiente projetado contínuo.

Definição 2.2. Dado $\delta \in \mathbb{R}_{+}^{m}$, o gradiente projetado contínuo no ponto z é dado por:

$$
g_{P}(z, \delta)=P_{\Omega(z, \delta)}(z-\nabla f(z))-z,
$$

$\operatorname{com} \Omega(z, \delta)=\left\{x \in \mathbb{R}^{n} \mid A x=b, l \leq x \leq u, c^{j} x \leq[d]_{j}, \forall j \in I(z, \delta)\right\}$ e $I(z, \delta)=$ $\left\{j \mid c^{j} z-[d]_{j} \geq-[\delta]_{j}\right\} . P_{\Omega(x, \delta)}(x)$ é a projeção ortogonal do ponto $x$ no conjunto $\Omega(x, \delta)$.

Note que $\Omega(z, \delta)$ é o conjunto das restrições de caixa e das restrições que são satisfeitas por igualdade ou que estão relativamente próximas de serem satisfeitas por igualdade e que, para $\delta=\infty$, temos $\Omega(z, \delta)=\Omega$.

GENLIN usa um algoritmo "irrestrito" com regiões de confiança ou com busca linear, apresentados em detalhes nas Seções 2.1.1 e 2.1.2, respectivamente, para encontrar o minimizador da função restrito à face da região factível definida pelo iterando atual, e o método do Gradiente Espectral Projetado Parcial (PSPG) para mudar de face, apresentado na Seção 2.2. 


\subsection{Algoritmos "irrestritos"}

Nesta seção, descrevemos o uso dos algoritmos "irrestritos" para encontrar o minimizador da função restrito a uma face.

Dada uma iteração $k$ e um iterando $x_{k}$, suponha, sem perda de generalidade, que as variáveis livres em $F_{I, J}$ são $\bar{x}=\left([x]_{1}, \ldots,[x]_{s}\right)^{T}$ e que as variáveis restantes estão fixas em $[\hat{x}]_{s+1}, \ldots,[\hat{x}]_{n}\left([\hat{x}]_{i} \in\left\{[l]_{i},[u]_{i}\right\}\right.$, para $\left.s+1 \leq i \leq n\right)$. Definamos $\bar{f}(\bar{x})=f\left([x]_{1}, \ldots,[x]_{s},[\hat{x}]_{s+1}, \ldots,[\hat{x}]_{n}\right), \bar{\Omega}=\left\{x \in \mathbb{R}^{s} \mid(x, \hat{x})^{T} \in F_{I, J}\right\}$, $\bar{A}$ e $\bar{b}$ correspondentes às restrições ativas de $\bar{\Omega}$ em $x_{k}$.

Usemos um algoritmo "irrestrito" para resolver o seguinte problema:

$$
\begin{array}{ll}
\text { Minimizar } & \bar{f}(x) \\
\text { sujeita a } & \bar{A} x=\bar{b},
\end{array}
$$

onde $x \in \mathbb{R}^{s}, \bar{A} \in \mathbb{R}^{t \times s}$ e $\bar{b} \in \mathbb{R}^{t}$.

Ao trabalhar com o problema (2.3), trataremos apenas do caso em que a matriz $\bar{A}$ possui linhas linearmente independentes. Isso é razoável na prática, pois quando alguma linha de $\bar{A}$ depende linearmente de outra, ou a linha em questão pode ser eliminada sem nenhuma alteração da solução de (2.3) ou a região viável é vazia, não havendo solução para o problema. Considere $Z \in \mathbb{R}^{s \times(s-t)}$ e $Y \in \mathbb{R}^{s \times t}$ as matrizes cujas colunas formam, respectivamente, uma base para espaço nulo e para a imagem do espaço gerado pelas linhas de $\bar{A}$. Assim, temos que $\bar{A} Z=0$ e $\bar{A} Y$ é não-singular.

Note que, uma vez escolhidas as matrizes $Z$ e $Y$, todo vetor $x$ em $\mathbb{R}^{s}$ possui uma única representação da forma

$$
x=Y x_{y}+Z x_{z}
$$

com $x_{y} \in \mathbb{R}^{t}$ e $x_{z} \in \mathbb{R}^{(s-t)}$. Em particular, tomando $x^{*}$ solução de (2.3), temos $x^{*}=Y x_{y}^{*}+Z x_{z}^{*}$. Como $x^{*}$ é viável, vale que

$$
\bar{A} x^{*}=\bar{A}\left(Y x_{y}^{*}+Z x_{z}^{*}\right)=\bar{b}
$$


Como $\bar{A} Z=0$, temos

$$
x_{y}^{*}=(\bar{A} Y)^{-1} \bar{b}
$$

pois $\bar{A} Y$ é não singular.

Assim, a solução $x^{*}$ do problema (2.3) (assim como qualquer ponto viável de (2.3)) pode ser vista como a soma de uma solução particular para o sistema linear $\bar{A} x=\bar{b}$ (fazendo-se, por exemplo, $x_{z}^{*}=0$ ) e de um ponto no núcleo do espaço gerado pelas linhas de $\bar{A}$ (ou seja, $Z x_{z}^{*}$ ). Desta forma, reduzimos o problema restrito (2.3) para um problema irrestrito de $s-t$ variáveis, dado por

$$
\operatorname{Minimizar} \bar{f}_{1}\left(x_{z}\right)=\bar{f}\left(Y(\bar{A} Y)^{-1} \bar{b}+Z x_{z}\right) \text {. }
$$

Note que o gradiente e a Hessiana de $\bar{f}_{1}$ são dados por

$$
\nabla \bar{f}_{1}\left(x_{z}\right)=Z^{T} \nabla \bar{f}(x)
$$

e

$$
\nabla^{2} \bar{f}_{1}\left(x_{z}\right)=Z^{T} \nabla^{2} \bar{f}(x) Z
$$

Formularemos, então, um algoritmo para resolver o problema (2.4) que gera uma sequência de pontos viáveis. Para manter a viabilidade de cada ponto gerado pelo algoritmo, a cada iteração $k$ partimos de um ponto $x_{k}$ viável e calculamos um passo $p_{k}$ tal que

$$
\bar{A} p_{k}=0
$$

Desta forma, temos que o ponto $x_{k+1}=x_{k}+\alpha_{k} p_{k}$ é viável, pois

$$
\bar{A} x_{k+1}=\bar{A}\left(x_{k}+\alpha_{k} p_{k}\right)=\bar{A} x_{k}+\alpha_{k} \bar{A} p_{k}=\bar{A} x_{k}=\bar{b}
$$

já que $x_{k}$ também é viável. Note que

$$
\bar{A} p_{k}=0 \Longleftrightarrow p_{k}=Z p^{z}
$$

para algum vetor $p^{z} \in \mathbb{R}^{(s-t)}$.

Nas Seções 2.1.1 e 2.1.2 apresentamos um algoritmo "irrestrito" com re- 
giões de confiança e com busca linear, respectivamente, para resolver o problema (2.4).

\subsubsection{Algoritmo "irrestrito" com regiões de confiança}

Nesta seção, apresentamos uma iteração de um algoritmo "irrestrito" baseado em regiões de confiança introduzido em Andretta et al. (2005), adaptado ao caso em que o problema original possui restrições lineares (veja Andretta (2008)).

Algoritmo 2.1 Iteração de um algoritmo "irrestrito" com regiões de confiança

Seja $k$ a iteração atual. Sejam $\bar{\Omega}$ e $\bar{f}(x)$ como definidos anteriormente. Dados $\bar{A} \in \mathbb{R}^{t \times s}, x_{k}$ tais que $\bar{A} x_{k}=\bar{b}, Z \in \mathbb{R}^{s \times(s-t)}$ tal que $\bar{A} Z=0, \Delta_{\text {min }}>0 \mathrm{e}$ $\Delta_{k} \geq \Delta_{\min }$, faça:

Passo 1. Se $Z^{T} \nabla \bar{f}\left(x_{k}\right)=0$ então pare e devolva $x_{k}$ como solução declarando "Ponto estacionário de primeira ordem".

Passo 2. Calcule $\Delta_{\text {borda }}$ a distância de $x_{k}$ à borda da face.

Se $\Delta_{\text {borda }}<2 \Delta_{\text {min }}$ então faça uma iteração do método SPG,

(Birgin et al. $(2000,2001)$ ), restrito a $F_{I, J}$ (face atual) para calcular $x_{k+1}$ e pare.

Passo 3. Calcule $s_{k}$ uma solução aproximada de

$$
\begin{array}{rlrl} 
& \text { Minimizar } & \varphi(w) & \equiv \frac{1}{2} w^{T} Z^{T} \nabla^{2} \bar{f}\left(x_{k}\right) Z w+\left(Z^{T} \nabla \bar{f}\left(x_{k}\right)\right)^{T} w \\
\text { sujeita a } & \|w\| & \leq \Delta_{k} .
\end{array}
$$

Passo 4. Se $\varphi\left(s_{k}\right)=0$ então pare e devolva $x_{k}$ como solução declarando "Ponto estacionário de segunda ordem". Senão, calcule $p_{k}=Z s_{k}$.

Passo 5. Se $x_{k}+p_{k}$ não pertence à face atual então calcule

$$
\alpha_{\max }=\max \left\{\alpha \in[0,1] \mid\left[x_{k}, x_{k}+\alpha p_{k}\right] \subset \bar{\Omega}\right\} .
$$

Se $\bar{f}\left(x_{k}+\alpha_{\max } p_{k}\right)<\bar{f}\left(x_{k}\right)$ então faça $x_{k+1}=x_{k}+\alpha_{\max } p_{k}$ e pare declarando "Iterando na borda". Senão faça 


$$
\Delta_{k}=\Delta_{\min }+0.7\left(\frac{\Delta_{\text {borda }}}{1+\sigma}-\Delta_{\text {min }}\right)
$$

e volte para o Passo 3.

Passo 6. Calcule $\rho_{k}=\frac{\bar{f}\left(x_{k}+p_{k}\right)-\bar{f}\left(x_{k}\right)}{\varphi\left(s_{k}\right)}$.

Passo 7. Se $\rho_{k} \leq 0.1$ então faça $\Delta_{k}=\Delta \in\left[0.1\left\|p_{k}\right\|, 0.9\left\|p_{k}\right\|\right]$ e volte para o Passo 3.

Passo 8. Faça $x_{k+1}=x_{k}+p_{k}$.

Passo 9. Escolha $\Delta_{k+1} \geq \Delta_{\min }$.

\section{Observações:}

- No Passo 1, utiliza-se o critério $\left\|Z^{T} \nabla f\left(x_{k}\right)\right\|_{\infty} \leq \epsilon$.

- No Passo 3, $s_{k}$ é calculado usando-se o algoritmo Moré-Sorensen (Moré e Sorensen (1983)) para minimização de quadrática em bolas (com parâmetro $\sigma$ ).

- No Passo 4, utiliza-se o teste $\left|\varphi\left(p_{k}\right)\right| \leq \epsilon_{\varphi}$.

- No Passo 7, calcula-se $\Delta_{k}=0.25\left\|p_{k}\right\|$.

- No Passo 9, o cálculo de $\Delta_{k+1}$ é feito da seguinte maneira:

$$
\begin{aligned}
& \Delta= \begin{cases}0.25\left\|p_{k}\right\|_{2}, & \text { se } \rho_{k} \leq 0.25 \\
2 \Delta_{k}, & \text { se } \rho_{k} \geq 0.5 \text { e }\left|\left\|p_{k}\right\|_{2}-\Delta_{k}\right| \leq 10^{-5} \\
\Delta_{k}, & \text { caso contrário, }\end{cases} \\
& \Delta_{k+1}=\max \left\{\Delta_{\min }, \Delta\right\} .
\end{aligned}
$$

- Define-se $\Delta_{0}$ como $\Delta_{0}=\max \left\{\Delta_{\min }, 100 \max \left\{1,\left\|x_{0}\right\|\right\}\right\}$.

Na Seção 2.1.3 apresentaremos como pode ser calculada a matriz $Z$. Assim a descrição do Algoritmo 2.1 se torna completa. 


\subsubsection{Algoritmo "irrestrito" com busca linear}

Nesta seção, apresentamos uma outra possibilidade para definir uma iteração de um algoritmo "irrestrito" para resolver o problema (2.4). O Algoritmo 2.2 é baseado em um método de Newton truncado com busca linear.

Algoritmo 2.2 Iteração de um algoritmo "irrestrito" com busca linear

Seja $k$ a iteração atual. Seja $\bar{\Omega}$ e $\bar{f}(x)$ como definido anteriormente. Dados $\bar{A} \in \mathbb{R}^{t \times s}, x_{k}$ tal que $\bar{A} x_{k}=\bar{b}, Z \in \mathbb{R}^{s \times(s-t)}$ tal que $\bar{A} Z=0, \theta \in(0,1) \mathrm{e}$ $\gamma \in(0,1)$.

Passo 1. Se $Z^{T} \nabla \bar{f}\left(x_{k}\right)=0$ então pare e devolva $x_{k}$ como solução declarando "Ponto estacionário de primeira ordem".

Passo 2. Calcule um vetor não nulo $p^{z} \in \mathbb{R}^{s-t}$ que satisfaça

$$
\left(Z^{T} \nabla \bar{f}\left(x_{k}\right)\right)^{T} p^{z} \leq-\theta\left\|Z^{T} \nabla \bar{f}\left(x_{k}\right)\right\|\left\|p^{z}\right\| .
$$

Calcule a direção $p_{k}=Z p^{z}$.

Passo 3. Calcule $\alpha_{\max }=\max \left\{\alpha \in[0,1] \mid\left[x_{k}, x_{k}+\alpha p_{k}\right] \subset \bar{\Omega}\right\}$.

Se $\alpha_{\max }<1$ e $\bar{f}\left(x_{k}+\alpha_{\max } p_{k}\right)<\bar{f}\left(x_{k}\right)$, então

faça $x_{k+1}=x_{k}+\alpha_{\max } p_{k}$ e pare declarando "Iterando na borda".

Passo 4. Calcule $\alpha_{k}<\alpha_{\max }$ para o qual valha

$$
\bar{f}\left(x_{k}+\alpha_{k} p_{k}\right) \leq \bar{f}\left(x_{k}\right)+\gamma \alpha_{k}\left(Z^{T} \nabla \bar{f}\left(x_{k}\right)\right)^{T} p^{z} .
$$

Passo 5. Faça $x_{k+1}=x_{k}+\alpha_{k} p_{k}$.

\section{Observações:}

- No Passo 1, utiliza-se o critério $\left\|Z^{T} \nabla f\left(x_{k}\right)\right\|_{\infty} \leq \epsilon$.

- No Passo 2, $p^{z}$ é calculado utilizando um método de gradientes conjugados para resolver o sistema Newtoniano

$$
B_{k} p^{z}=-Z^{T} \nabla \bar{f}\left(x_{k}\right)
$$

onde $B_{k}$ é uma aproximação de $Z^{T} \nabla^{2} \bar{f} Z$ no ponto $x_{k}$. A cada iteração de 
gradientes conjugados, toma-se o cuidado de satisfazer a condição (2.5). Quando esta não é válida, toma-se o ponto calculado na iteração anterior.

- No Passo 4, utiliza-se "backtracking" com interpolação quadrática unidimensional para calcular $\alpha_{k}$. A quadrática interpolante é dada por $q(w)$, $\operatorname{com} q(0)=\bar{f}\left(x_{k}\right), q\left(\alpha_{k}\right)=\bar{f}\left(x_{k}+\alpha_{k} p_{k}\right)$ e $\nabla q(0)=\nabla \bar{f}\left(x_{k}\right)^{T} p_{k}$. Definimos $\bar{\alpha}_{\text {novo }}$ como o minimizador da função $q$ e utilizamos $\bar{\alpha}_{\text {novo }}$ para calcular $\alpha_{k}$.

Passo 4.1. Faça $\alpha_{\text {tent }} \leftarrow \alpha_{\max }$.

Passo 4.2. Se $\bar{f}\left(x_{k}+\alpha_{\text {tent }} p_{k}\right) \leq \bar{f}\left(x_{k}\right)+\gamma \alpha_{\text {tent }}\left(Z^{T} \nabla \bar{f}\left(x_{k}\right)\right)^{T} p^{z}$, então faça $\alpha_{k}=\alpha_{\text {tent }}$ e pare.

Passo 4.3. Calcule

$$
\begin{gathered}
\bar{\alpha}_{\text {novo }}=-\frac{\alpha_{\text {tent }}^{2}\left(Z^{T} \nabla \bar{f}\left(x_{k}\right)\right)^{T} p^{z}}{2\left(\bar{f}\left(x_{k}+\alpha_{\text {tent }} p_{k}\right)-\bar{f}\left(x_{k}\right)-\alpha_{\text {tent }}\left(Z^{T} \nabla \bar{f}\left(x_{k}\right)\right)^{T} p^{z}\right)} . \\
\text { Se } \bar{\alpha}_{\text {novo }} \geq 0.1 \alpha_{\text {tent }} \text { e } \bar{\alpha}_{\text {novo }} \leq 0.9 \alpha_{\text {tent }}, \\
\quad \text { então faça } \alpha_{\text {novo }} \leftarrow \bar{\alpha}_{\text {novo }} \text { Senão, faça } \alpha_{\text {novo }} \leftarrow 0.5 \alpha_{\text {tent }} . \\
\text { Faça } \alpha_{\text {tent }} \leftarrow \alpha_{\text {novo }} \text { e volte para o Passo } 4.2 .
\end{gathered}
$$

Para que a descrição do Algoritmo 2.2 esteja completa, restará apenas mostrar como calcular a matriz $Z$, o que será feito na Seção 2.1.3.

\subsubsection{Cálculo da base do núcleo}

Apresentamos aqui duas técnicas que podem ser utilizadas para calcular a matriz $Z$ usada nos Algoritmos 2.1 e 2.2.

\subsubsection{Fatoração $L Q$}

A primeira ideia para o cálculo da matriz $Z$ é o uso da fatoração LQ da matriz $\bar{A}$. Nesta fatoração, que gasta $O\left(s t^{2}\right)$ operações, temos uma matriz ortogonal $Q \in \mathbb{R}^{s \times s}$ tal que

$$
\bar{A} Q=\left(\begin{array}{ll}
L & 0
\end{array}\right)
$$


onde $L \in \mathbb{R}^{t \times t}$ é triangular inferior. Como estamos supondo que as linhas da matriz $\bar{A}$ são linearmente independentes, a matriz $L$ é não-singular.

Escrevendo a matriz $Q$ como $Q=\left[Q_{1} ; Q_{2}\right]$, com $Q_{1} \in \mathbb{R}^{s \times t}$ e $Q_{2} \in \mathbb{R}^{s \times(s-t)}$, temos que

$$
\bar{A} Q_{1}=L \text { e } \bar{A} Q_{2}=0
$$

É fácil ver que as colunas de $Q_{1}$ e $Q_{2}$ formam, respectivamente, uma base ortogonal para a imagem e o núcleo do espaço gerado pelas linhas de $\bar{A}$. Assim, uma escolha natural para a matriz $Z$ é $Q_{2}$.

Note que, se utilizarmos técnicas do tipo Newton para calcular o passo $p^{z}$ (Passo 2 do Algoritmo 2.2), é importante levar em conta o condicionamento da Hessiana reduzida $Z^{T} \nabla^{2} \bar{f}\left(x_{k}\right) Z$.

Neste caso, temos que

$$
\operatorname{cond}\left(Z^{T} \nabla^{2} \bar{f}\left(x_{k}\right) Z\right) \leq \operatorname{cond}\left(\nabla^{2} \bar{f}\left(x_{k}\right)\right)(\operatorname{cond}(Z))^{2}
$$

Assim, como este limitante é justo (pode valer a igualdade), se o número de condição da matriz $Z$ fosse grande, a matriz Hessiana reduzida $Z^{T} \nabla^{2} \bar{f}\left(x_{k}\right) Z$ poderia ser mau-condicionada, independentemente do número de condição da matriz Hessiana $\nabla^{2} \bar{f}\left(x_{k}\right)$. No entanto, utilizando a fatoração LQ para determinar $Z$, temos $\operatorname{cond}(Z)=1$. Ou seja,

$$
\operatorname{cond}\left(Z^{T} \nabla^{2} \bar{f}\left(x_{k}\right) Z\right) \leq \operatorname{cond}\left(\nabla^{2} \bar{f}\left(x_{k}\right)\right)
$$

o que mostra que, neste caso, o condicionamento do problema (2.4) não é pior que o condicionamento do problema (2.3). Portanto, uma grande vantagem do uso desta técnica é o fato de que esta escolha de $Z$ não piora o condicionamento do problema de encontrar uma solução para o problema (2.4).

Para formar a matriz $Q$ usando a fatoração LQ, é necessário calcular o produto de transformações ortogonais usadas para triangularizar a matriz $\bar{A}$. Na implementação de métodos que usam a matriz $Z$ somente em produtos matrizvetor, pode não ser eficiente calcular a matriz $Q$ (e, conseqüentemente, $Z$ ) explicitamente, principalmente quando o número de restrições é pequeno. Neste caso é mais interessante armazenar as transformações ortogonais de forma com- 
pacta e aplicá-las ao vetor apropriado para calcular o produto matriz-vetor sempre que necessário.

\subsubsection{Redução de variáveis}

Apesar das vantagens do uso da fatoração LQ para o cálculo da matriz $Z$, esta é uma operação computacionalmente custosa e que não aproveita uma eventual esparsidade de $\bar{A}$. Uma alternativa é a técnica de redução de variáveis.

Tomando a matriz $\bar{A}$ com linhas linearmente independentes, podemos particioná-la em

$$
\bar{A}=\left(\begin{array}{ll}
V & U
\end{array}\right),
$$

onde $V \in \mathbb{R}^{t \times t}$ é não singular e $U \in \mathbb{R}^{t \times(s-t)}$. Usando esta partição de $\bar{A}$, a matriz $Z$ pode ser definida como

$$
Z=\left(\begin{array}{c}
-V^{-1} U \\
I
\end{array}\right)
$$

onde $I$ é a matriz identidade $(s-t) \times(s-t)$. Note que

$$
\bar{A} Z=\left(\begin{array}{ll}
V & U
\end{array}\right)\left(\begin{array}{c}
-V^{-1} U \\
I
\end{array}\right)=-V V^{-1} U+U=0
$$

como desejamos.

Geralmente a matriz $Z$, tal como descrita acima, não é armazenada explicitamente. Produtos matriz-vetor envolvendo $Z$ e $Z^{T}$ podem ser obtidos resolvendo sistemas lineares envolvendo $V$ e $V^{T}$. A grande dificuldade desta técnica é encontrar uma maneira eficiente de particionar $\bar{A}$ de forma que a matriz $V$ escolhida fique relativamente bem-condicionada.

\subsection{Método do Gradiente Espectral Projetado Parcial}

Quando GENLIN, apresentado em Andretta et al. (2010), decide mudar de face, ou seja, decide mudar o conjunto de restrições ativas corrente, usamos o método do Gradiente Espectral Projetado Parcial (PSPG) (inspirado no método do Gradiente Espectral Projetado SPG, apresentado em Birgin et al. (2000, 2001)), para definir qual será a nova face a ser trabalhada. Em outras 
palavras, para escolher quais restrições devem entrar no conjunto das restrições ativas e quais devem sair deste conjunto.

O método do Gradiente Espectral Projetado é usado para resolver o seguinte problema:

$$
\begin{array}{ll}
\text { Minimizar } & f(x) \\
\text { sujeita a } & x \in \Omega,
\end{array}
$$

onde $f$ é uma função em $\mathcal{C}^{1}, x \in \mathbb{R}^{n}$ e $\Omega$ é um conjunto convexo fechado. No nosso caso, $\Omega=\left\{x \in \mathbb{R}^{n} \mid A x=b, C x \leq d, l \leq x \leq u\right\}$.

A ideia do algoritmo é a seguinte: na iteração $k$, calculamos uma direção $p_{k}$ e damos um passo $\alpha_{k}$ tal que $x_{k+1}=x_{k}+\alpha_{k} p_{k} \in \Omega$ satisfaça a condição de Armijo. A direção $p_{k}$ é calculada com base na projeção ortogonal de $-\lambda_{k}^{\mathrm{PSPG}} \nabla f\left(x_{k}\right)$ no conjunto $\Omega\left(x_{k}, \delta\right)$, onde $\lambda_{k}^{\mathrm{PSPG}}$ (chamado de passo espectral) é um escalar calculado a partir de informações fornecidas pelo ponto atual $x_{k}$ e pelo ponto $x_{k-1}$ da iteração anterior.

Denotamos por $\Omega^{0}$ o conjunto de pontos interiores de $\Omega$. Os passos de uma iteração de PSPG são dados a seguir.

\section{Algoritmo 2.3 Iteração de PSPG}

Seja $k$ a iteração atual. Suponha que $\nabla f\left(x_{k}\right) \neq 0$. Dados $x_{k} \in \Omega^{0}, 0<$ $\lambda_{\min }^{\mathrm{PSPG}}<\lambda_{\max }^{\mathrm{PSPG}}<\infty, \gamma \in(0,1), \delta_{k} \geq \delta_{\min }>0$.

Passo 1. Cálculo do $\lambda_{k}^{\mathrm{PSPG}}$

Se $k=0$ ou $\left(x_{k}-x_{k-1}\right)^{T}\left(\nabla f\left(x_{k}\right)-\nabla f\left(x_{k-1}\right)\right) \leq 0$, então faça

$$
\begin{aligned}
& \lambda_{k}^{\mathrm{PSPG}}=1 \text {. Senão, calcule } s_{k}=x_{k}-x_{k-1}, \\
& y_{k}=\nabla f\left(x_{k}\right)-\nabla f\left(x_{k-1}\right) \text { e faça } \\
& \lambda_{k}^{\mathrm{PSPG}}=\min \left\{\lambda_{\max }^{\mathrm{PSPG}}, \max \left\{\lambda_{\min }^{\mathrm{PSPG}}, \frac{s_{k}^{T} s_{k}}{s_{k}^{T} y_{k}}\right\}\right\} .
\end{aligned}
$$

Passo 2. Calcule $p_{k}^{\mathrm{PSPG}}=P_{\Omega\left(x_{k}, \delta_{k}\right)}\left(x_{k}-\lambda_{k}^{\mathrm{PSPG}} \nabla f\left(x_{k}\right)\right)-x_{k}$.

Passo 3. Calcule $\alpha_{\max }=\max \left\{\alpha \in[0,1] \mid\left[x_{k}, x_{k}+\alpha p_{k}^{\mathrm{PSPG}}\right] \subset \Omega\right\}$. 
Passo 4. Faça $\alpha_{\text {tent }} \leftarrow \alpha_{\text {max }}$.

Passo 5. Se $f\left(x_{k}+\alpha_{\text {tent }} p_{k}\right) \leq f\left(x_{k}\right)+\gamma \alpha_{\text {tent }} \nabla f\left(x_{k}\right)^{T} p_{k}^{\mathrm{PSPG}}$,

então faça $\alpha_{k}=\alpha_{\text {tent }}$ e termine a iteração com

$x_{k+1}=x_{k}+\alpha_{k} p_{k}^{\mathrm{PSPG}}$.

Senão, escolha $\alpha_{\text {novo }} \in\left[0,1 \alpha_{\text {tent }}, 0.9 \alpha_{\text {tent }}\right]$, faça $\alpha_{\text {tent }} \leftarrow \alpha_{\text {novo }}$

e repita o Passo 5.

\section{Observações:}

- No Passo 5, escolhemos $\alpha_{\text {novo }}$ usando interpolação quadrática uni-dimensional com salvaguardas. A quadrática interpolante é dada por $q(w)$, $\operatorname{com} q(0)=f\left(x_{k}\right), q\left(\alpha_{k}\right)=f\left(x_{k}+\alpha_{k} p_{k}^{\mathrm{PSPG}}\right)$ e $\nabla q(0)=\nabla f\left(x_{k}\right)^{T} p_{k}^{\mathrm{PSPG}}$. Definimos $\bar{\alpha}_{\text {novo }}$ como o minimizador da função $q$ com salvaguardas.

Passo 5.1. Faça

$$
\bar{\alpha}_{\text {novo }}=-\frac{\alpha_{\text {tent }}^{2} \nabla f\left(x_{k}\right)^{T} p_{k}^{\mathrm{PSPG}}}{2\left(f\left(x_{k}+\alpha_{\text {tent }} p_{k}^{P S P G}\right)-f\left(x_{k}\right)-\alpha_{\text {tent }} \nabla f\left(x_{k}\right)^{T} p_{k}^{\mathrm{PSPG}}\right)} .
$$

Passo 5.2. Salvaguarda de $\alpha_{\text {novo }}$

Se $\bar{\alpha}_{\text {novo }} \geq 0.1 \alpha_{\text {tent }}$ e $\bar{\alpha}_{\text {novo }} \leq 0.9 \alpha_{\text {tent }}$ então faça

$\alpha_{\text {novo }} \leftarrow \bar{\alpha}_{\text {novo }}$. Senão, faça $\alpha_{\text {novo }} \leftarrow 0.5 \alpha_{\text {tent }}$.

- Definimos $\lambda_{\min }^{\mathrm{PSPG}}=10^{-10}$ e $\lambda_{\max }^{\mathrm{PSPG}}=10^{10}$.

Note que, para $\delta=\infty$, o algoritmo 2.3 se reduz ao método SPG, apresentado em Birgin et al. (2000, 2001).

Definido o método PSPG para mudar de face e dados os algoritmos "irrestritos" que podem ser usados para encontrar o minimizador da função restrito à face atual, podemos agora escrever o método de restrições ativas GENLIN para resolver o problema (1). 


\subsection{Método de restrições ativas para problemas com res- trições lineares}

Agora que dispomos de todas as ferramentas necessárias, descrevemos o método de restrições ativas para problemas com restrições lineares chamado GENLIN.

Algoritmo 2.4 Método de restrições ativas para problemas com restrições lineares (GENLIN)

$\operatorname{Dados} x_{0} \in \mathbb{R}^{n}$ e $\delta_{1} \geq \delta_{\min }>0$.

Passo 1. Cálcule $x_{1}=P_{\Omega}\left(x_{0}\right)$.

Se é possível calcular $x_{1}$, então faça $k \leftarrow 1$ e $b d \leftarrow 0$. Senão, pare declarando "Problema inviável".

Passo 2. Atingiu borda por 20 iterações seguidas. Sai da face.

Se $b d=20$ então vá para o Passo 3. Senão, vá para o Passo 4.

Passo 3. Critério de convergência.

Se $g_{P}\left(x_{k}, \delta_{k}\right)=0$, então pare e devolva $x_{k}$ como solução.

Senão, use uma iteração do Algoritmo 2.3 para calcular $x_{k+1}$, faça $b d \leftarrow 0, k \leftarrow k+1$ e vá para o Passo 4 .

Passo 4. $x_{k}$ é um minimizador na face.

Se $P_{S_{I, J}}\left(-\nabla f\left(x_{k}\right)\right)=0$ então volte para o Passo 3.

Passo 5. Permanece na mesma face

Calcule $x_{k+1} \in \bar{\Omega}$ usando o Algoritmo 2.1 ou 2.2 para resolver o problema (2.3) com $x_{k}$ como ponto inicial.

Passo 6. Ponto $x_{k+1}$ está na borda.

Se o algoritmo "irrestrito" usado no Passo 5 termina sua execução com $x_{k+1}$ declarando "Iterando na borda", então faça $b d \leftarrow b d+1$. Senão, faça $b d \leftarrow 0$.

Passo 7. Faça $k \leftarrow k+1$ e volte para o Passo 2 . 
Para que GENLIN esteja totalmente definido, mostraremos na Seção 2.4 como é calculada a projeção de um ponto nas restrições lineares.

\subsection{Projeção}

Note que projetar um ponto $\tilde{x} \in \mathbb{R}^{n}$ em um conjunto $\tilde{\Omega}=\{x \in \mathbb{R} \mid A x=$ $b, \tilde{C} x \leq \tilde{d}, l \leq x \leq u\}$ significa encontrar um ponto $\tilde{x}^{*}$ que seja solução do seguinte problema:

$$
\begin{array}{ll}
\text { Minimizar } \quad & \frac{1}{2}\|x-\tilde{x}\|_{2}^{2} \equiv \frac{1}{2} x^{T} I x-\tilde{x}^{T} x \\
\text { sujeita a } \quad & A x=b \\
& \tilde{C} x \leq \tilde{d} \\
& l \leq x \leq u
\end{array}
$$

onde $I$ é a matriz identidade em $\mathbb{R}^{n \times n}, \tilde{C} \in \mathbb{R}^{\tilde{m} \times n}$ e $\tilde{d} \in \mathbb{R}^{\tilde{m}}$ correspondem às restrições lineares de desigualdade utilizadas na projeção.

Como este é um problema de programação quadrática convexa, existem algoritmos conhecidos que podem ser utilizados para resolver (2.6). Um algoritmo clássico para a resolução deste problema é o proposto em Goldfarb e Idnani (1983).

Uma outra maneira de calcular a projeção seria considerar o problema dual de (2.6), dado por

$$
\begin{array}{ll}
\text { Minimizar } & \frac{1}{2} y^{T} \tilde{A} \tilde{A}^{T} y+(\tilde{b}-\tilde{A} \tilde{x})^{T} y \\
\text { sujeita a } & y_{i} \geq 0, \quad i=r+1, r+2, \ldots, r+\tilde{m}+n l+n u
\end{array}
$$

onde $\tilde{A} \in \mathbb{R}^{(r+\tilde{m}+n l+n u) \times n}, \tilde{A}=\left[\begin{array}{c}A \\ \tilde{C} \\ -I_{l} \\ I_{u}\end{array}\right], \tilde{b} \in \mathbb{R}^{r+\tilde{m}+n l+n u}, \tilde{b}=\left[\begin{array}{c}b \\ \tilde{d} \\ -\ell \\ u\end{array}\right], \mathcal{I}_{l}=$ $\left\{i \mid[x]_{i} \geq[l]_{i},[l]_{i} \in \mathbb{R}\right\}, \mathcal{I}_{u}=\left\{i \mid[x]_{i} \leq[u]_{i},[u]_{i} \in \mathbb{R}\right\}, n l=\left|\mathcal{I}_{l}\right|, n u=\left|\mathcal{I}_{u}\right|$, $I_{l} \in \mathbb{R}^{n l \times n}$ é a matriz formada pelas linhas da identidade com índice em $\mathcal{I}_{l}$ e $I_{u} \in \mathbb{R}^{n u \times n}$ é a matriz formada pelas linhas da identidade com índice em $\mathcal{I}_{u}$. 
Assim, qualquer algoritmo para minimização de quadráticas em caixas poderia ser utilizado para encontrar a solução $y^{*}$ do problema (2.7). Dado $y^{*}$, a solução primal (ou seja, o ponto $\tilde{x}^{*}$ projeção de $\tilde{x}$ em $\tilde{\Omega}$ ) é dada por $\tilde{x}^{*}=\tilde{x}-\tilde{A}^{T} y^{*}$.

Embora sofra de problemas de condicionamento, esta formulação é mais interessante para o caso esparso, para o qual há menos implementações de métodos para resolver o problema de programação quadrática (2.6). No caso de problemas esparsos, é necessário um método para resolver (2.7) que não fatore a matriz Hessiana do problema.

Usaremos os métodos de programação quadrática para problemas com restrições de caixa apresentados no Capítulo 1 para resolver o problema de projeção, usando a formulação (2.7).

\subsection{Teoria de convergência}

Para simplificar a teoria de convergência de GENLIN, vamos escrever o problema (1) como

$$
\begin{array}{ll}
\text { Minimizar } & f(x) \\
\text { sujeita a } & x \in \Omega=\left\{x \in \mathbb{R}^{n} \mid C_{i}(x) \leq 0, i=1, \ldots, p\right\}
\end{array}
$$

com

$$
C_{i}(x)=\left(a^{i}\right)^{T} x-[b]_{i}, a^{i}=\left(a_{1}^{i}, \ldots, a_{n}^{i}\right)^{T} .
$$

Vamos supor que $\Omega$ é compacto. Claramente, os problemas (1) e (2.8) são equivalentes. Dado $I \subset\{1, \ldots, p\}$, definimos $F_{I}$ de maneira análoga a $F_{I, J}$, ou seja,

$$
F_{I}=\left\{x \in \Omega \mid C_{i}(x)=0 \text { se e somente se } i \in I\right\} .
$$

Note que $\Omega$ é a união dos conjuntos $F_{I}$, para $I \subset\{1, \ldots, p\}$. Ainda, $F_{I} \neq F_{J}$ implica que $F_{I} \cap F_{J}=\emptyset$. O menor espaço afim que contém a face não-vazia $F_{I}$ será denotado por $V_{I}$ e o subespaço linear paralelo a $V_{I}$ será denotado por $S_{I}$.

Dados $z \in \Omega$ e $\delta \in[0, \infty]^{p}$, definimos

$$
\Omega(z, \delta)=\left\{x \in \mathbb{R}^{n} \mid C_{i}(x) \leq 0 \text { para todo } i \in I(z, \delta)\right\}
$$


onde

$$
I(z, \delta)=\left\{i \mid C_{i}(z) \geq-[\delta]_{i}\right\} .
$$

Claramente, $\Omega \subset \Omega(z, \delta)$ e $\Omega(z, \infty)=\Omega$.

A seguir, apresentaremos uma propriedade que será útil para provar a convergência de GENLIN.

Propriedade 2.1. Se $z \in \Omega$ satisfaz as condições KKT de (2.8) então z também satisfaz as condições KKT de

$$
\begin{array}{ll}
\text { Minimizar } & f(x) \\
\text { sujeita a } & x \in \Omega(z, \delta),
\end{array}
$$

para todo $\delta \geq 0$. Reciprocamente, se z satisfaz as condições KKT de (2.9) para algum $\delta \geq 0$, então z satisfaz as condições KKT de (2.8).

Para a prova, veja o Apêndice A.

Dado $z \in \Omega$, definimos

$$
g_{P}(z)=P_{\Omega}(z-\nabla f(z))-z
$$

e

$$
g_{P}(z, \delta)=P_{\Omega(z, \delta)}(z-\nabla f(z))-z .
$$

Além disso, para todo $\sigma>0$, definimos

$$
g_{P}(z, \delta, \sigma)=P_{\Omega(z, \delta)}(z-\sigma \nabla f(z))-z .
$$

Finalmente, se $z \in F_{I} \subset \Omega$, definimos

$$
g_{S}(z)=P_{S_{I}}(-\nabla f(z))=-Z^{T} \nabla f(z) .
$$

Note que vale que

$$
g_{P}(z)=g_{P}(z, \infty)=g_{P}(z, \infty, 1)
$$

e

$$
g_{P}(z, \delta)=g_{P}(z, \delta, 1) .
$$


Dados $\delta \in[0, \infty)^{p}$ e $\sigma>0$, satisfazer as condições KKT para $z \in \Omega$ é equivalente a $g_{P}(z, \delta, \sigma)=0$. Ainda, se $\left[\delta_{k}\right]_{i} \geq \delta_{\min }>0$ para todo $i=1, \ldots, p$, $k \in \mathbb{N}, z_{k} \rightarrow z$ e $z$ é um ponto KKT, temos que $\left\|g_{P}\left(z_{k}, \delta_{k}\right)\right\| \rightarrow 0$. Isso justifica o uso de $\left\|g_{P}\left(z_{k}, \delta_{k}\right)\right\|$ como critério de parada do método.

Para provar a convergência do Algoritmo 2.4, precisamos definir e apresentar o teorema de convergência de um algoritmo auxiliar. Primeiramente, vejamos a definição do método de Métrica Variável Inexata (Birgin et al. (2003)). Este método é usado para resolver o problema de minimizar uma função $f(x)$ sujeita a $x \in \Omega$, com $\Omega$ fechado e convexo, e $f$ com derivadas parciais contínuas em um conjunto aberto que contém $\Omega$.

Defina $\mathcal{M}$ o conjunto das matrizes em $\mathbb{R}^{n \times n}$ definidas positivas tais que, para $B \in \mathcal{M},\|B\| \leq L$ e $\left\|B^{-1}\right\| \leq L$, para algum $L$. Defina $g_{k}=\nabla f\left(x_{k}\right)$.

\section{Algoritmo 2.5 Iteração do método de Métrica Variável Inexata}

Sejam $k$ a iteração atual e $x_{0} \in \Omega$ um ponto inicial arbitrário. Dados $\eta \in$ $(0,1], \gamma \in(0,1), 0<\sigma_{1}<\sigma_{2}<1, M$ um inteiro positivo, $x_{k} \in \Omega, B_{k} \in \mathcal{M}$.

Passo 1. Cálculo da direção de busca

Considere o subproblema

$$
\begin{array}{ll}
\text { Minimizar } & Q_{k}(d) \\
\text { sujeita a } & x_{k}+d \in \Omega,
\end{array}
$$

com

$$
Q_{k}(d)=\frac{1}{2} d^{T} B_{k} d+g_{k}^{T} d
$$

Seja $\bar{d}_{k}$ o minimizador de (2.16). Seja $d_{k}$ tal que $x_{k}+d_{k} \in \Omega$ e

$$
Q_{k}\left(d_{k}\right) \leq \eta Q_{k}\left(\bar{d}_{k}\right)
$$

Se $d_{k}=0$, então pare declarando "Ponto estacionário".

Passo 2. Cálculo do tamanho do passo.

$$
\text { Faça } \alpha=1 \text { e } f_{\max }=\max \left\{f\left(x_{k-j+1}\right) \mid 1 \leq j \leq \min \{k+1, M\}\right\} \text {. }
$$


Passo 3. Teste de decréscimo da função.

Se

$$
f\left(x_{k}+\alpha d_{k}\right) \leq f_{\max }+\gamma \alpha g_{k}^{T} d_{k},
$$

então faça $\alpha_{k}=\alpha, x_{k+1}=x_{k}+\alpha_{k} d_{k}$ e pare. Senão, escolha

$\alpha_{\text {novo }} \in\left[\sigma_{1} \alpha, \sigma_{2} \alpha\right]$, faça $\alpha \leftarrow \alpha_{\text {novo }}$ e repita o Passo 3 .

O Lema 2.1 e o Teorema 2.1, apresentados a seguir, são o Lema 2.1 e o Teorema 2.1 de Birgin et al. (2003), respectivamente.

Lema 2.1. O método de Métrica Variável Inexata é bem-definido.

Teorema 2.1. Suponha que o conjunto de nível $\left\{x \in \Omega \mid f(x) \leq f\left(x_{0}\right)\right\}$ é limitado. Então, ou o método de Métrica Variável Inexata pára em algum ponto estacionário $x_{k}$, ou todo ponto limite de sequência gerada é estacionário.

Vejamos agora a definição de uma iteração da versão monótona do método SPG (Birgin et al. (2000, 2001)), que será usado na prova da convergência de GENLIN.

Algoritmo 2.6 Iteração de SPG monótono

Seja $\hat{\Omega} \subset \mathbb{R}^{n}$ um conjunto convexo fechado. Suponha que $\alpha \in(0,1), 0<$ $\sigma_{\min }<\sigma_{\max }<\infty$. Seja $x_{0} \in \hat{\Omega}$ um ponto inicial arbitrário. Seja $k$ a iteração atual. Dados $x_{k} \in \hat{\Omega}, \sigma_{k} \in\left[\sigma_{\min }, \sigma_{\max }\right]$.

Passo 1. Cálculo da direção de busca.

Calcule

$$
p_{k}=P_{\hat{\Omega}}\left(x_{k}-\frac{1}{\sigma_{k}} \nabla f\left(x_{k}\right)\right)-x_{k} .
$$

Se $p_{k}=0$, pare com $x_{k}$ declarando "Ponto estacionário".

Passo 2. Cálculo do tamanho do passo.

Escolha $t_{\text {inicial }} \geq t_{\text {min }}$ e faça $t \leftarrow t_{\text {inicial }}$. 
Passo 3. Teste de decréscimo da função.

Se

$$
f\left(x_{k}+t p_{k}\right) \leq f\left(x_{k}\right)+\alpha t \nabla f\left(x_{k}\right)^{T} p_{k},
$$

então faça $x_{k+1}$ tal que $f\left(x_{k+1}\right) \leq f\left(x_{k}+t p_{k}\right)$ e pare. Senão, escolha $t_{\text {novo }} \in[0.1 t, 0.9 t]$, faça $t \leftarrow t_{\text {novo }}$ e repita o Passo 3 .

Teorema 2.2. Seja $\hat{\Omega}$ convexo e fechado. Suponha que a sequência gerada pelo Algoritmo 2.6 seja limitada. Então

- Se o Algoritmo 2.6 não pára em $x_{k} \in \hat{\Omega}$, então $x_{k+1}$ é bem-definido.

- Se o Algoritmo 2.6 pára em $x_{k} \in \hat{\Omega}$, então $x_{k}$ é um ponto estacionário do problema

$$
\begin{array}{ll}
\text { Minimizar } & f(x) \\
\text { sujeita a } & x \in \hat{\Omega} .
\end{array}
$$

- Se $x^{*}$ é um ponto limite da sequência gerada pelo Algoritmo 2.6, então $x^{*}$ é um ponto KKT de (2.19). Mais ainda,

$$
\lim _{k \rightarrow+\infty}\left\|p_{k}\right\|=\lim _{k \rightarrow+\infty}\left\|P_{\hat{\Omega}}\left(x_{k}-\nabla f\left(x_{k}\right)\right)-x_{k}\right\|=0
$$

Prova: Note que o método SPG monótono se reduz ao Método de Métrica Variável Inexata apresentado anteriormente. Basta tomar $M=1$ e $Q_{k}(d)=$ $\frac{\|d\|^{2} \sigma_{k}}{2}+g_{k}^{T} d$. Assim, a prova segue diretamente do Lema 2.1 e do Teorema 2.1.

Agora podemos provar a convergência de GENLIN (Algoritmo 2.4). O algoritmo "irrestrito" a ser utilizado no Passo 5 do Algoritmo 2.4 admite várias implementações. Básicamente, é um algoritmo para minimização irrestrita que pára quando atinge a borda da região viável. Como visto na Seção 2.1, utilizamos os algoritmos "irrestritos" com regiões de confiança ou com busca linear. Para provar a convergência de GENLIN, estes algoritmos devem satisfazer as seguintes hipóteses.

\section{Hipótese 1}

- Se $x_{k+1}$ é calculado pelo algoritmo "irrestrito" e $x_{k} \in F_{I}$, então $x_{k+1} \in \bar{F}_{I}$ e $f\left(x_{k+1}\right) \leq f\left(x_{k}\right)$. 
- Se $x_{k+j} \in F_{I}$ é calculado pelo algoritmo "irrestrito" para todo $j \geq 1$, existe $j$ tal que $\left\|g_{S}\left(x_{k+j}\right)\right\|_{\infty} \leq \epsilon$, em que $g_{S}(x)$ está definido em (2.13).

Teorema 2.3. O Algoritmo 2.1 satisfaz Hipótese 1.

Prova: Pela construção do algoritmo, se ele pára em $x_{k}$, vale a Hipótese 1. Basta verificar o caso em que o algoritmo gera uma sequência infinita $\left\{x_{k}\right\}$. Neste caso, ou temos infinitas iterações de SPG ou temos infinitas iterações de regiões de confiança. Se são realizadas infinitas iterações de SPG, temos que o ponto limite da sequência $\left\{x_{k}\right\}$ é um ponto estacionário. Se são realizadas infinitas iterações de regiões de confiança, por argumentos clássicos de minimização irrestrita, temos também que o ponto limite da sequência $\left\{x_{k}\right\}$ é um ponto estacionário. Ou seja, em ambos os casos, para $k$ suficientemente grande, $\left\|g_{S}\left(x_{k+j}\right)\right\|_{\infty} \leq \epsilon$.

Teorema 2.4. O Algoritmo 2.2 satisfaz Hipótese 1.

Prova: Pela construção do algoritmo, se ele pára em $x_{k}$, vale a Hipótese 1 . Basta verificar o caso em que o algoritmo gera uma sequência infinita $\left\{x_{k}\right\}$. Neste caso, ou temos infinitas iterações irrestritas de Newton truncado, ou o algoritmo atinge a borda e realiza infinitas iterações de backtracking para obter um ponto onde haja decréscimo suficiente da função. Em ambos os casos, argumentos clássicos de minimização irrestrita garantem que o ponto limite da sequência $\left\{x_{k}\right\}$ é um ponto estacionário. Ou seja, em ambos os casos, para $k$ suficientemente grande, $\left\|g_{S}\left(x_{k+j}\right)\right\|_{\infty} \leq \epsilon$.

Teorema 2.5. Suponha que GENLIN (Algoritmo 2.4) seja aplicado ao problema (2.8). Então:

- Para todo $k=0,1,2, \ldots$, se GENLIN não termina em $x_{k}$, então $x_{k+1}$ está bem-definido.

- A sequência $\left\{x_{k}\right\}$ gerada pelo GENLIN termina em um número finito de iterações em um ponto no qual $\left\|g_{P}\left(x_{k}, \delta_{k}\right)\right\|_{\infty} \leq \epsilon$.

Prova: A primeira parte da tese segue da Hipótese 1 e dos Teoremas 2.3 e 2.4. Vamos provar a segunda parte. Suponha, por contradição, que GENLIN 
gera infinitas iterações. Consideramos dois casos:

1. Há infinitas iterações de PSPG.

2. Há um número finito de iterações de PSPG.

Considere o primeiro caso. Seja $K \subset \mathbb{N}$ o conjunto de índices $k$ tal que $x_{k+1}$ é computado pelo método PSPG. Como o número de faces $F_{I}$ é finito, existem $I \subset\{1, \ldots, p\}$ e $K_{1}$ um subconjunto infinito de $K$ tal que $x_{k} \in F_{I}$ para todo $k \in K_{1}$. Note que o número de subconjuntos diferentes $\Omega\left(x_{k}, \delta_{k}\right)$ também é finito e, portanto, existem $\hat{\Omega}$ e $K_{2}$, um subconjunto infinito de $K_{1}$, tais que

$$
\Omega\left(x_{k}, \delta_{k}\right)=\hat{\Omega} \quad \text { para todo } k \in K_{2} .
$$

Portanto, para todo $k \in K_{2}$,

$$
p_{k}=P_{\hat{\Omega}}\left(x_{k}-\lambda_{k}^{\mathrm{PSPG}} \nabla f\left(x_{k}\right)\right)-x_{k} .
$$

Suponha, sem perda de generalidade, que $\hat{\Omega}$ seja definido pelas desigualdades

$$
\left(a^{i}\right)^{T} x-[b]_{i} \leq 0 \quad \text { para todo } i=1, \ldots, q .
$$

Seja $i \in\{q+1, \ldots, p\}$. Então,

$$
\left(a^{i}\right)^{T} x_{k}-[b]_{i}<-\left[\delta_{k}\right]_{i}
$$

Suponha que $\alpha \geq 0$ seja tal que

$$
\left(a^{i}\right)^{T}\left(x_{k}+\alpha p_{k}\right)-[b]_{i}=0 .
$$

Então,

$$
\alpha=\frac{[b]_{i}-\left(a^{i}\right)^{T} x_{k}}{\left(a^{i}\right)^{T} p_{k}} .
$$

Portanto,

$$
\alpha>\frac{\left[\delta_{k}\right]_{i}}{\left\|a^{i}\right\|\left\|p_{k}\right\|}
$$

Como $\Omega$ é compacto, a sequência $\left\{x_{k}\right\}$ é limitada e, então, a sequência $\left\{p_{k}\right\}$ 
também é limitada. Ou seja $\left\|p_{k}\right\| \leq c$ para todo $k$. Portanto,

$$
\alpha>\frac{\left[\delta_{k}\right]_{i}}{\left\|a^{i}\right\| c} \geq \frac{\min \left\{\left[\delta_{k}\right]_{i}\right\}}{\max \left\{\left\|a^{i}\right\|\right\} c} \equiv \alpha_{\min } .
$$

Isso implica que o primeiro ponto da forma $x_{k}+\alpha_{\max } p_{k}$ que é testado no método PSPG está longe de $x_{k}+\alpha_{\min } p_{k}$ para $\alpha_{\min }>0$. Portanto, como $f\left(x_{k+1}\right)<f\left(x_{k}\right)$ para todo $k$, a sequência $\left\{x_{k}\right\}_{k \in K_{2}}$ pode ser pensada, depois de renomeados seus índices, como sendo gerada pelo Algoritmo 2.6 aplicado à minimização de $f$ em $\hat{\Omega}$ (tomando $t_{\text {inicial }}=\alpha_{\max }$ ). Portanto, qualquer ponto limite $x^{*}$ da sequência é um ponto estacionário. Como $\Omega \subset \hat{\Omega}$, isso implica que $x^{*}$ é um ponto estácionario de (2.8). Então, $\lim _{k \rightarrow K_{2}}\left\|g_{P}\left(x_{k}, \delta_{k}\right)\right\|=0$. Portanto, para $k \in K_{2}$ suficientemente grande, temos que $\left\|g_{P}\left(x_{k}, \delta_{k}\right)\right\|_{\infty} \leq \epsilon$ e GENLIN teria parado em $x_{k}$. Isso contradiz a hipótese de que $\left\{x_{k}\right\}$ é infinita.

No caso em que há um número finito de iterações de PSPG, temos que, para $k$ suficientemente grande, todas as iterações são internas. Mais ainda, depois de um número finito de iterações, todos os iterandos pertencem à mesma face $F_{I}$. Pela Hipótese 1 , isso implica que existe $k$ tal que $\left\|g_{S}\left(x_{k}\right)\right\|_{\infty} \leq \epsilon$. Portanto, nesta iteração devemos ter $\left\|g_{P}\left(x_{k}, \delta_{k}\right)\right\|_{\infty} \leq \epsilon$, caso contrário o próximo iterando seria obtido por PSPG.

\subsection{Detalhes de implementação}

Implementamos, em Fortran 77, duas novas versões de GENLIN, que chamamos de GENLIN-GPCG e GENLIN-NQC.

A versão original de GENLIN (que chamamos de, simplesmente, GENLIN) já estava disponível e implementada em Fortran 77. Ela utiliza, para calcular as projeções, a formulação primal (2.6). Para resolver este problema, utiliza a rotina QL, implementada por Powell (1983), modificada por Schittkowski (2004) e apresentada em Goldfarb e Idnani (1983). Esta rotina calcula as projeções usando álgebra linear densa e é ideal para problemas de pequeno e médio porte.

Para calcular as projeções, as duas novas versões de GENLIN utilizam a formulação (2.7). GENLIN-GPCG utiliza a implementação GPCG, apresen- 
tada na Seção 1.1, para resolver os problemas de projeção de forma esparsa. Já GENLIN-NQC utiliza a implementação NQC, apresentada na Seção (1.2), para resolver os problemas de projeção, também de forma esparsa.

Todos os parâmetros padrão de GENLIN foram mantidos. O cálculo da matriz $Z$ (veja Seção 2.1.3) é feito usando fatoração LQ e o algoritmo "irrestrito" usado é o algoritmo de busca linear com Newton truncado (Algoritmo 2.2). Para detalhes, veja Andretta et al. (2010).

A precisão da viabilidade das caixas e das restrições lineares $\epsilon_{\text {viab }}$ nos três algoritmos é de $10^{-8}$. A precisão na rotina $\mathrm{QL}$ é de $\epsilon_{\mathrm{QL}}=\left(\epsilon_{\mathrm{viab}}\right)^{1.25}$, e a precisão nos algoritmos GPCG e NQC é de $\epsilon_{\mathrm{GPCG}}=\epsilon_{\mathrm{NQC}}=10^{-8}$. O número máximo de iterações é de 5000, para os três algoritmos.

Como a matriz $\tilde{A} \tilde{A}^{T}$ da formulação (2.7) pode não ser definida positiva, optamos por resolver o problema

$$
\begin{array}{ll}
\text { Minimizar } & \frac{1}{2} y^{T}\left(\tilde{A} \tilde{A}^{T}+I \gamma\right) y+(\tilde{b}-\tilde{A} \tilde{x})^{T} y \\
\text { sujeita a } & y_{i} \geq 0, \quad i=r+1, r+2, \ldots, r+\tilde{m}+2 n,
\end{array}
$$

$\operatorname{com}[\gamma]_{i}=\max \left\{10^{-16}, \gamma^{0}[d]_{i}\right\}$, tal que $\left(\tilde{A} \tilde{A}^{T}+I \gamma\right)$ é definida positiva, sendo $d$ a diagonal da matriz $\tilde{A} \tilde{A}^{T}$ e $10^{-16} \leq \gamma^{0} \leq 10^{-5}$.

Nas Seções 2.6.1 e 2.6.2, apresentamos detalhes da implementação de GENLINGPCG e GENLIN-NQC, respectivamente.

\subsubsection{Detalhes da implementação de GENLIN-GPCG}

A rotina GPCG possui um parâmetro de saída $\mathrm{INFO}_{\mathrm{GPCG}}$, que pode ter os seguintes valores:

- $\mathrm{INFO}_{\mathrm{GPCG}}=0$ : Sucesso. O critério de convergência $\left\|\nabla_{\Omega_{\square}} q(x)\right\|_{2} \leq$ $\epsilon_{\mathrm{GPCG}}$ foi satisfeito;

- $\mathrm{INFO}_{\mathrm{GPCG}}=1$ : Número máximo de iterações foi atingido;

- $\mathrm{INFO}_{\mathrm{GPCG}}=2$ : Não foi encontrado um $\alpha$ que satisfaça a condição de decréscimo suficiente da busca projetada (1.17); 
- $\mathrm{INFO}_{\mathrm{GPCG}}=3$ : Progresso insuficiente;

- $\mathrm{INFO}_{\mathrm{GPCG}}=4$ : Matriz Hessiana não é definida positiva;

- $\mathrm{INFO}_{\mathrm{GPCG}}=5$ : Valor da função tende a menos infinito. Neste caso, valor da função menor do que $-10^{20}$.

Independentemente do critério de convergência satisfeito por GPCG, verificamos a viabilidade do ponto obtido. Nosso objetivo na projeção é obter um ponto viável com tolerância $\epsilon_{\text {viab}}$, ou seja, estamos interessados em um ponto $x$ que satisfaça $\rho_{\text {viab }}(x) \leq \epsilon_{\text {viab }}$, com

$$
\rho_{\text {viab }}(x)=\max _{\substack{i=1, \ldots, m \\ j=1, \ldots, n}}\left\{0,\|A x-b\|,[C x-d]_{i},[l-x]_{j},[x-u]_{j}\right\}
$$

Para que isso aconteça, utilizamos o seguinte procedimento, usando $\epsilon_{\mathrm{GPCG}}=$ $10^{-8}, \gamma^{0}=10^{-16}$ e $R=\emptyset$.

Passo 1. Primeira tentativa.

Chame a rotina GPCG com $\epsilon_{\mathrm{GPCG}}$ e $\gamma^{0}$. Seja $\bar{x}$ o ponto projetado e $\mathrm{INFO}_{\mathrm{GPCG}}$ o status de saída da rotina. Faça $R=R \cup\{\bar{x}\}$.

Se $\rho_{\text {viab }}(\bar{x}) \leq \epsilon_{\text {viab}}$, então pare e devolva $\bar{x}$ como solução declarando "Sucessso na projeção".

Se $\mathrm{INFO}_{\mathrm{GPCG}}=0$, então vá para o Passo 2. Senão, vá para o

Passo 3.

Passo 2. Tentativa de satisfazer $\rho_{\mathrm{viab}}(x) \leq \epsilon_{\mathrm{viab}}$ utilizando tolerância mais justa.

$$
\begin{aligned}
& \text { Se } \epsilon_{\mathrm{GPCG}}>10^{-16}, \\
& \text { faça } \epsilon_{\mathrm{GPCG}}=10^{-2} \epsilon_{\mathrm{GPCG}} \text {. } \\
& \text { Se } \gamma^{0}<10^{-8} \text {, faça } \gamma^{0}=10 \gamma^{0} \text { e repita o Passo } 1 . \\
& \text { Senão, vá para o Passo } 4 .
\end{aligned}
$$

Passo 3. Tentativa com $\gamma^{0}$ maior ou tolerância menos restrita. 
Se $\gamma^{0}<10^{-8}$, então faça $\gamma^{0}=10 \gamma^{0}$ e repita o Passo 1 .

Senão,

faça $\epsilon_{\mathrm{GPCG}}=10 \epsilon_{\mathrm{GPCG}}$.

Chame a rotina GPCG com $\epsilon_{\mathrm{GPCG}}$ e $\gamma^{0}$. Seja $\bar{x}$ o ponto projetado e $\mathrm{INFO}_{\mathrm{GPCG}}$ o status de saída da rotina.

Faça $R=R \cup\{\bar{x}\}$.

Se $\rho_{\text {viab }}(\bar{x}) \leq \epsilon_{\text {viab }}$, então pare e devolva $\bar{x}$ como solução declarando "Sucessso na projeção".

Passo 4. Tratamento de perda de viabilidade.

Seja $\hat{x}=\operatorname{argmin}_{x \in R}\left\{\rho_{\text {viab }}(x)\right\}$. Se $\rho_{\text {viab }}(\hat{x}) \leq \sqrt{\epsilon_{\text {viab }}}$, então pare e devolva $\hat{x}$ como solução declarando "Perda parcial de viabilidade." Senão, pare declarando "Falha na projeção".

\subsubsection{Detalhes da implementação de GENLIN-NQC}

A rotina $\mathrm{NQC}$ possui um parâmetro de saída $\mathrm{INFO}_{\mathrm{NQC}}$, que pode ter os seguintes valores:

- $\mathrm{INFO}_{\mathrm{NQC}}=0$ : Sucesso. O critério de convergência $\| P_{\Omega_{\square}}\left(x_{k}-\nabla q\left(x_{k}\right)\right)-$ $x_{k} \|<\epsilon_{\mathrm{NQC}}$ foi satisfeito;

- $\mathrm{INFO}_{\mathrm{NQC}}=4$ : Ciclo encontrado.

- $\mathrm{INFO}_{\mathrm{NQC}}<0$ : Problema na matriz Hessiana.

Independentemente do critério de convergência satisfeito por NQC, verificamos a viabilidade do ponto obtido. Nosso objetivo na projeção é obter um ponto viável com tolerância $\epsilon_{\mathrm{viab}}$, ou seja, estamos interessados em um ponto $x$ que satisfaça $\rho_{\text {viab }}(x) \leq \epsilon_{\text {viab }}$, com $\rho_{\text {viab }}(x)$ como em (2.21).

Para que isso aconteça, utilizamos o seguinte procedimento, usando $\epsilon_{\mathrm{NQC}}=$ $10^{-8}, \gamma^{0}=10^{-16}$ e $R=\emptyset$.

Passo 1. Primeira tentativa. 
Chame a rotina NQC com $\epsilon_{\mathrm{NQC}}$ e $\gamma^{0}$. Sejam $\bar{x}$ o ponto projetado e $\mathrm{INFO}_{\mathrm{NQC}}$ o status de saída da rotina.

Se $\mathrm{INFO}_{\mathrm{NQC}}$ é menor que zero ou igual a 4 , então

Se $\gamma^{0}<10^{-5}$, faça $\gamma^{0}=10 \gamma^{0}$ e repita o Passo 1 .

Senão,

faça $R=R \cup\{\bar{x}\}$.

Se $\rho_{\text {viab }}(\bar{x}) \leq \epsilon_{\text {viab }}$, então pare e devolva $\bar{x}$ como solução

declarando "Sucessso na projeção".

Se $\mathrm{INFO}_{\mathrm{NQC}}=0$, então vá para o Passo 2. Senão, vá para o

Passo 3.

Passo 2. Tentativa de satisfazer $\rho_{\mathrm{viab}}(x) \leq \epsilon_{\mathrm{viab}}$ utilizando tolerância mais justa.

Se $\epsilon_{\mathrm{GPCG}}>10^{-16}$, faça $\epsilon_{\mathrm{GPCG}}=10^{-2} \epsilon_{\mathrm{GPCG}}$. Repita o Passo 1.

Senão, vá para o Passo 4.

Passo 3. Tentativa de tolerância menos restrita.

Faça $\epsilon_{\mathrm{NQC}}=10 \epsilon_{\mathrm{NQC}}$. Chame a rotina NQC com $\epsilon_{\mathrm{NQC}}$ e $\gamma^{0}$. Seja $\bar{x}$

o ponto projetado e $\mathrm{INFO}_{\mathrm{NQC}}$ o status de saída da rotina.

Faça $R=R \cup\{\bar{x}\}$.

Se $\rho_{\text {viab }}(\bar{x}) \leq \epsilon_{\text {viab }}$, então pare e devolva $\bar{x}$ como solução declarando "Sucessso na projeção".

Passo 4. Tratamento de perda de viabilidade.

Seja $\hat{x}=\operatorname{argmin}_{x \in R}\left\{\rho_{\text {viab }}(x)\right\}$. Se $\rho_{\text {viab }}(\hat{x}) \leq \sqrt{\epsilon_{\text {viab }}}$, então pare e devolva $\hat{x}$ como solução declarando "Perda parcial de viabilidade." Senão, pare declarando "Falha na projeção".

\subsection{Resultados numéricos}

Nesta seção, apresentamos os resultados numéricos obtidos ao aplicar GENLIN, GENLIN-GPCG e GENLIN-NQC na resolução dos problemas da coleção CUTEr com até 500 variáveis, restrições de caixa e de 1 a 2000 restrições line- 
ares, totalizando 133 problemas. Vale observar que, apesar de GPCG e NQC terem sido desenvolvidos para resolver problemas esparsos, GENLIN utiliza álgebra linear densa em outros pontos de seu código, o que nos leva a solucionar problemas de menor porte.

Os códigos foram compilados em gfortran, usando o sistema operacional Ubuntu 11.10. Os experimentos numéricos foram realizados em um PC com Processador $2^{\text {a }}$ Geração Intel Core i3 2310M, 2.1 GHz, 4GB de memória.

O critério de convergência usado nestes experimentos é que a norma infinito do gradiente projetado no ponto deve ser menor ou igual a $\epsilon_{\text {otim }}=10^{-8}$ $\left(\left\|g_{P}\left(x_{k}, \delta_{k}\right)\right\|_{\infty} \leq 10^{-8}\right)$. Foi determinado um número máximo de iterações (200000) e um número máximo de avaliações de função (1000000).

Os problemas NASH, MODEL, ARGLALE, ARGLBLE, ARGLCLE e LINCONT são inviáveis e, de fato, nenhuma das três versões de GENLIN encontrou um ponto inicial viável. Portanto, estes problemas foram eliminados dos experimentos. Daqui em diante, serão considerados somente os 127 problemas restantes, apresentados nas Tabelas 2.1-2.3. Nestas tabelas, Nome é o nome do problema; $n$ é o número de variáveis; $n l$ é o número de variáveis com limitante inferior; nu é o número de variáveis com limitante superior; mleq é o número de restrições lineares de igualdade; e mlin é o número de restrições lineares de desigualdade.

Os resultados obtidos pela aplicação de GENLIN, GENLIN-GPCG e GENLIN-NQC para a resolução dos problemas da coleção CUTEr podem ser encontrados nas Tabelas 2.4-2.6, 2.7-2.9 e 2.10-2.12, respectivamente. Nestas tabelas, Tempo é o tempo de execução em segundos; $k$ é o número de iterações; $n_{f}$ é o número de avaliações da função; $n_{g}$ é o número de avaliações do gradiente; $n_{h}$ é o número de avaliações da Hessiana; $n_{c g}$ é o número de iterações do Gradiente Conjugado; $n_{s p g}$ é o número de iterações do PSPG; $n_{f_{s p g}}$ é o número de avaliações da função realizadas nas iterações do PSPG; $n_{n t}$ é o número de iterações do Newton truncado; $n_{f_{n t}}$ é o número de avaliações da função realizadas nas iterações do Newton truncado; iproj é o número de projeções feitas; $f\left(x^{*}\right)$ é o valor da função no ponto final $x^{*}$ obtido pelos algoritmos; cnorm é a norma das restrições lineares; e gpsupn é a norma do gradiente projetado descontínuo. 
Nas Tabelas 2.4-2.6, ql é o número de vezes que é chamada a rotina QL; nas Tabelas 2.7-2.9, GPCG e iGPCG são o número de vezes que é chamada a rotina GPCG e o número total de iterações feitas nessa rotina, respectivamente; nas Tabelas 2.10-2.12, NQC e iNQC são o número de vezes que é chamada a rotina NQC e o número total de iterações feitas nessa rotina, respectivamente.

Nas Tabelas 2.4-2.12, inf é igual a zero, se o critério de convergência é satisfeito; igual a 2 , se o método parou por progresso insuficiente; igual a 4 , se o método parou porque o valor da função tende a menos infinito, neste caso, o valor da função fica menor do que $-10^{20}$; igual a 6 , se o tamanho do passo é muito pequeno na busca linear; igual a 7 , se o número máximo de iterações é atingido; igual a 10, se o método parou porque a direção não pode ser calculada devido a erros ocorridos durante seus cálculos; igual a -30, se o método parou em um ponto viável, mas um problema ocorreu na projeção; igual a -40, se o método parou em um ponto inviável; igual a -90, se ocorreu um erro ao avaliar a função; igual a 99, se o tempo de execução foi excedido. O tempo máximo de execução nos três métodos é de 2000 segundos.

Devido a problemas de condicionamento na formulação (2.7), muitos problemas não satisfazem o critério de convergência ao aplicar GENLIN-GPCG e GENLIN-NQC nos problemas apresentados nas Tabelas 2.1-2.3.

Em 14 problemas, a aplicação do método GENLIN-GPCG resulta em pontos inviáveis. Em 12 problemas, a aplicação do método GENLIN-NQC resulta em pontos inviáveis. Em todos os problemas, a aplicação do método GENLIN resulta em pontos viáveis. Pode-se ver que GENLIN é mais robusto.

A fim de comparar os resultados obtidos ao aplicar GENLIN, GENLINGPCG e GENLIN-NQC, dizemos que dois valores de função $f_{1}$ e $f_{2}$ são iguais se

$$
\left|f_{1}-f_{2}\right| \leq \max \left\{10^{-10}, 10^{-6} \min \left\{\left|f_{1}\right|,\left|f_{2}\right|\right\}\right\}
$$

A seguir comparamos o desempenho de GENLIN-GPCG com GENLINNQC. Para fazer uma comparação melhor, excluímos os 14 problemas para os quais o método GENLIN-GPCG pára com inf igual a -40, os 7 problemas 
para os quais GENLIN-NQC pára com inf igual a -40 e os 5 problemas para os quais o método GENLIN-NQC pára por tempo de execução excedido, em um total de 19 problemas distintos. Os 2 problemas para os quais o método GENLIN-GPCG pára por tempo de execução excedido não são excluídos ao fazer as comparações, já que o método pára em um ponto viável. Ao comparar GENLIN-GPCG com GENLIN-NQC nos 108 problemas restantes, podemos ver que:

- Em 17 problemas, GENLIN-GPCG obteve valor de função melhor.

- Em 7 problemas, GENLIN-NQC obteve valor de função melhor.

- nos 84 problemas restantes, o valor da função é igual ao aplicar ambos os métodos.

Estamos interessados em comparar, basicamente, o tempo gasto por cada método e a qualidade da solução encontrada por cada método. Para visualizar melhor esta comparação, construímos os gráficos do perfil de desempenho, que podem ser vistos nas Figuras 2.1 e 2.2. Nestes gráficos, usando o tempo como medida de desempenho, se um ponto da curva correspondente a um dos métodos vale $I$ no eixo das abscissas e $J$ no eixo das ordenadas, significa que este método resolve $(J \times 100) \%$ dos problemas em um tempo menor ou igual a $I$ vezes o tempo mais rápido. Os pontos mais significativos do gráfico são o mais à esquerda e o mais à direita. À esquerda medimos a eficiência dos métodos (verificamos a porcentagem de problemas para os quais cada método é o mais rápido) e à direita medimos a robustez (verificamos a porcentagem de problemas que cada método foi capaz de resolver).

Para a construção dos gráficos das Figuras 2.1 e 2.2, comparamos os tempos de execução de GENLIN-GPCG e de GENLIN-NQC. Para os gráficos da Figura 2.1, quando um dos dois métodos encontra valor de função maior para um mesmo problema, consideramos que este método levou tempo infinito para encontrar a solução. Para os gráficos da Figura 2.2, consideramos apenas os problemas para os quais ambos os métodos chegaram ao mesmo valor de função.

Considerando o número de problemas para os quais GENLIN-GPCG e GENLIN-NQC param em pontos inviáveis (14 problemas para GENLIN-GPCG 
e 12 problemas para GENLIN-NQC) poderiamos dizer que GENLIN-NQC é um pouco mais robusto que GENLIN-GPCG. Mas, observando os gráficos das Figuras 2.1 e 2.2, também poderiamos dizer que GENLIN-GPCG é um pouco mais robusto que GENLIN-NQC, já que achou melhores soluções em um número maior de problemas (Robustez - GENLIN-GPCG 89,81\%, GENLIN-NQC $80,55 \%)$.

GENLIN-NQC é um pouco mais eficiente que GENLIN-GPCG tanto quando penalizamos os métodos que encontraram pontos com valores de função piores (Eficiência - GENLIN-GPCG 70,37\%, GENLIN-NQC 75\%), como quando usamos na comparação apenas os problemas em que os valores de função são iguais (Eficiência - GENLIN-GPCG 72,36\%, GENLIN-NQC 92,10\%).

A seguir comparamos o desempenho de GENLIN-GPCG com GENLIN. Para fazer uma comparação melhor, excluímos os 14 problemas para os quais o método GENLIN-GPCG pára com inf igual a -40. Ao comparar GENLIN com GENLIN-GPCG nos 113 problemas restantes, podemos ver que:

- Em 10 problemas, GENLIN obteve valor de função melhor. Destes 10 problemas, só em 1 problema GENLIN-GPCG parou com inf igual a zero; nos 9 problemas restantes, GENLIN-GPCG parou em um ponto viável que não satisfaz o critério de convergência.

- Em 7 problemas, GENLIN-GPCG obteve valor de função melhor. É significativo dizer que nesses 7 problemas GENLIN-GPCG pára em um ponto viável, mas que não satisfaz o critério de convergência. Esses problemas são: SIPOW1M, OET3, LOTSCHD, KSIP e GMNCASE4, para os quais GENLIN-GPCG pára com inf igual a 2, e WATER e QPCBLEND, para os quais GENLIN-GPCG para com inf igual a -30 e inf igual a 6 , respectivamente.

- Nos 96 problemas restantes, o valor da função é igual ao aplicar ambos os métodos.

Estamos interessados em comparar, basicamente, o tempo gasto por GENLIN e GENLIN-GPCG e a qualidade da solução encontrada por cada método. Para visualizar melhor esta comparação, construímos os gráficos do perfil de desempenho, que podem ser vistos nas Figuras 2.3 e 2.4. Nestes gráficos, usa- 
mos o tempo como medida de desempenho.

Para a construção dos gráficos das Figuras 2.3 e 2.4, comparamos os tempos de execução de GENLIN e de GENLIN-GPCG. Para os gráficos da Figura 2.3, quando um dos dois métodos encontra valor de função maior para um mesmo problema, consideramos que este método levou tempo infinito para encontrar a solução. Para os gráficos da Figura 2.4, consideramos apenas os problemas para os quais ambos os métodos chegaram ao mesmo valor de função.

Observando os dados das Tabelas 2.4-2.6, 2.7-2.9 e os gráficos das Figuras 2.3 e 2.4, podemos notar que GENLIN é mais robusto (Robustez - GENLIN 100\%, GENLIN-GPCG 93,80\%) e mais eficiente que GENLIN-GPCG, tanto quando penalizamos os métodos que encontraram pontos com valores de função piores (Eficiência - GENLIN 96,46\%, GENLIN-GPCG 52,21\%), como quando usamos na comparação apenas os problemas em que os valores de função são iguais (Eficiência - GENLIN 96,26\%, GENLIN-GPCG 55,14\%).

A seguir, comparamos o desempenho de GENLIN com GENLIN-NQC. Para fazer uma comparação melhor, excluímos os 7 problemas para os quais o método GENLIN-NQC pára com inf igual a -40 e os 5 problemas para os quais pára por tempo de execução excedido. Ao comparar GENLIN com GENLINNQC nos 115 problemas restantes, podemos ver que:

- Em 20 problemas, GENLIN obteve valor de função melhor. Nestes 20 problemas, GENLIN-NQC parou com inf igual a -30.

- Em 4 problemas, GENLIN-NQC obteve valor de função melhor. Desses 4 problemas, GENLIN-NQC pára em 2 problemas (DEGENLPB e KSIP) com inf igual a 2 .

- Nos 91 problemas restantes, o valor da função é igual ao aplicar ambos os métodos.

Estamos interessados em comparar, o tempo gasto por cada método e a qualidade da solução encontrada por cada método. Para visualizar melhor esta comparação, construímos os gráficos do perfil de desempenho, que podem ser vistos nas Figuras 2.5-2.6. Nestes gráficos, usamos o tempo como medida de desempenho. 
Para a construção dos gráficos das Figuras 2.5-2.6, comparamos os tempos de execução de GENLIN e de GENLIN-NQC. Para os gráficos da Figura 2.5, quando um dos dois métodos encontra valor de função maior para um mesmo problema, consideramos que este método levou tempo infinito para encontrar a solução. Para os gráficos da Figura 2.6, consideramos apenas os problemas para os quais ambos os métodos chegaram ao mesmo valor de função.

Observando os dados das Tabelas 2.4-2.6, 2.10-2.12 e os gráficos das Figuras 2.5 e 2.6, podemos notar que GENLIN é mais robusto (Robustez - GENLIN 98,26\%, GENLIN-NQC 83,47\%) e mais eficiente que GENLIN-NQC, tanto quando penalizamos os métodos que encontraram pontos com valores de função piores (Eficiência - GENLIN 86,95\%, GENLIN-NQC 66,08\%), como quando usamos na comparação apenas os problemas em que os valores de função são iguais (Eficiência - GENLIN 86,17\%, GENLIN-NQC 78,72\%). 
Tabela 2.1: Problemas com apenas restrições lineares selecionados da coleção CUTEr

\begin{tabular}{|l|r|r|r|r|r|}
\hline Nome & $n$ & $n l$ & $n u$ & mleq & mlin \\
\hline EXTRASIM & 2 & 1 & 0 & 1 & 0 \\
\hline HS9 & 2 & 0 & 0 & 1 & 0 \\
\hline TAME & 2 & 2 & 0 & 1 & 0 \\
\hline HS21 & 2 & 2 & 2 & 0 & 1 \\
\hline HS35MOD & 2 & 2 & 0 & 0 & 1 \\
\hline HUBFIT & 2 & 1 & 0 & 0 & 1 \\
\hline LSQFIT & 2 & 1 & 0 & 0 & 1 \\
\hline BOOTH & 2 & 0 & 0 & 2 & 0 \\
\hline HIMMELBA & 2 & 0 & 0 & 2 & 0 \\
\hline SUPERSIM & 2 & 1 & 0 & 2 & 0 \\
\hline SIMPLLPA & 2 & 2 & 0 & 0 & 2 \\
\hline ZECEVIC2 & 2 & 2 & 2 & 0 & 2 \\
\hline HS24 & 2 & 2 & 0 & 0 & 2 \\
\hline SIMPLLPB & 2 & 2 & 0 & 0 & 3 \\
\hline PT & 2 & 0 & 0 & 0 & 501 \\
\hline SIPOW1M & 2 & 0 & 0 & 0 & 288 \\
\hline SIPOW1 & 2 & 0 & 0 & 0 & 288 \\
\hline SIPOW2M & 2 & 0 & 0 & 0 & 144 \\
\hline SIPOW2 & 2 & 0 & 0 & 0 & 144 \\
\hline HS28 & 3 & 0 & 0 & 1 & 0 \\
\hline HS62 & 3 & 3 & 3 & 1 & 0 \\
\hline HS35I & 3 & 3 & 3 & 0 & 1 \\
\hline HS35 & 3 & 3 & 0 & 0 & 1 \\
\hline HS36 & 3 & 3 & 3 & 0 & 1 \\
\hline HS37 & 3 & 3 & 3 & 0 & 1 \\
\hline STANCMIN & 3 & 3 & 0 & 0 & 2 \\
\hline ZANGWIL3 & 3 & 0 & 0 & 3 & 0 \\
\hline TFI2 & 3 & 0 & 0 & 0 & 101 \\
\hline TFI3 & 3 & 0 & 0 & 0 & 16 \\
\hline OET1 & 3 & 0 & 0 & 0 & 1002 \\
\hline HONG & 4 & 4 & 4 & 1 & 0 \\
\hline HS41 & 4 & 4 & 4 & 1 & 0 \\
\hline LIN & 4 & 4 & 4 & 2 & 0 \\
\hline HS76I & 4 & 4 & 4 & 0 & 2 \\
\hline HS76 & 4 & 4 & 0 & 0 & 2 \\
\hline S277-280 & 4 & 4 & 0 & 0 & 4 \\
\hline HS44NEW & 4 & 4 & 0 & 0 & 2 \\
\hline HS44 & 4 & 4 & 0 & 0 & 2 \\
\hline BIGGSC4 & 4 & 4 & 4 & 0 & 13 \\
\hline HATFLDH & 4 & 4 & 4 & 0 & 13 \\
\hline OET3 & 4 & 0 & 0 & 0 & 1002 \\
\hline SIPOW3 & 4 & 0 & 0 & 0 & 653 \\
\hline SIPOW4 & 4 & 0 & 0 & 0 & 2 \\
\hline
\end{tabular}


Tabela 2.2: Problemas com apenas restrições lineares selecionados da coleção CUTEr

\begin{tabular}{|l|r|r|r|r|r|}
\hline Nome & $n$ & $n l$ & nu & mleq & mlin \\
\hline HS48 & 5 & 0 & 0 & 2 & 0 \\
\hline HS49 & 5 & 0 & 0 & 2 & 0 \\
\hline BT3 & 5 & 0 & 0 & 3 & 0 \\
\hline HS50 & 5 & 0 & 0 & 3 & 0 \\
\hline HS51 & 5 & 0 & 0 & 3 & 0 \\
\hline HS52 & 5 & 0 & 0 & 3 & 0 \\
\hline HS53 & 5 & 5 & 5 & 3 & 0 \\
\hline LSNNODOC & 5 & 3 & 3 & 4 & 0 \\
\hline HS268 & 5 & 0 & 0 & 0 & 2 \\
\hline S268 & 5 & 0 & 0 & 0 & 2 \\
\hline HS86 & 5 & 5 & 0 & 0 & 2 \\
\hline EXPFITA & 5 & 0 & 0 & 0 & 2 \\
\hline EXPFITB & 5 & 0 & 0 & 0 & 3 \\
\hline EXPFITC & 5 & 0 & 0 & 0 & 12 \\
\hline HS54 & 6 & 6 & 6 & 1 & 0 \\
\hline HS55 & 6 & 6 & 2 & 6 & 0 \\
\hline PENTAGON & 6 & 0 & 0 & 0 & 15 \\
\hline HS21MOD & 7 & 4 & 4 & 0 & 1 \\
\hline EQC & 7 & 7 & 7 & 0 & 3 \\
\hline QCNEW & 7 & 7 & 7 & 0 & 3 \\
\hline QC & 7 & 7 & 7 & 0 & 1 \\
\hline DUALC2 & 7 & 7 & 7 & 1 & 228 \\
\hline HS105 & 8 & 8 & 8 & 0 & 1 \\
\hline AVGASA & 8 & 8 & 8 & 0 & 10 \\
\hline AVGASB & 8 & 8 & 8 & 0 & 10 \\
\hline DUALC5 & 8 & 8 & 8 & 1 & 277 \\
\hline DUALC8 & 8 & 8 & 8 & 1 & 502 \\
\hline DUALC1 & 9 & 9 & 9 & 1 & 214 \\
\hline HS112 & 10 & 10 & 0 & 3 & 0 \\
\hline ODFITS & 10 & 10 & 0 & 6 & 0 \\
\hline GENHS28 & 10 & 0 & 0 & 8 & 0 \\
\hline PORTFL1 & 12 & 12 & 12 & 1 & 0 \\
\hline PORTFL2 & 12 & 12 & 12 & 1 & 0 \\
\hline PORTFL3 & 12 & 12 & 12 & 1 & 0 \\
\hline PORTFL4 & 12 & 12 & 12 & 1 & 0 \\
\hline PORTFL6 & 12 & 12 & 12 & 1 & 0 \\
\hline LOTSCHD & 12 & 12 & 0 & 7 & 0 \\
\hline HS118 & 15 & 15 & 15 & 0 & 1 \\
\hline HS119 & 16 & 16 & 16 & 8 & 0 \\
\hline FCCU & 19 & 19 & 0 & 8 & 0 \\
\hline RES & 20 & 20 & 20 & 12 & 2 \\
\hline DEGENLPA & 20 & 20 & 20 & 15 & 0 \\
\hline DEGENLPB & 20 & 20 & 20 & 15 & 0 \\
\hline & & & & & \\
\hline
\end{tabular}


Tabela 2.3: Problemas com apenas restrições lineares selecionados da coleção CUTEr

\begin{tabular}{|l|r|r|r|r|r|}
\hline Nome & $n$ & $n l$ & $n u$ & mleq & mlin \\
\hline KSIP & 20 & 0 & 0 & 0 & 879 \\
\hline MAKELA4 & 21 & 0 & 0 & 0 & 40 \\
\hline WATER & 31 & 31 & 31 & 10 & 0 \\
\hline LOADBAL & 31 & 31 & 11 & 11 & 0 \\
\hline HIMMELBJ & 43 & 43 & 0 & 14 & 0 \\
\hline DALLASS & 46 & 46 & 46 & 31 & 0 \\
\hline AVION2 & 49 & 49 & 49 & 15 & 0 \\
\hline GOFFIN & 51 & 0 & 0 & 0 & 50 \\
\hline DUAL4 & 75 & 75 & 75 & 1 & 0 \\
\hline LINSPANH & 81 & 81 & 81 & 33 & 0 \\
\hline SPANHYD & 81 & 81 & 81 & 33 & 0 \\
\hline QPCBLEND & 83 & 83 & 0 & 43 & 23 \\
\hline QPNBLEND & 83 & 83 & 0 & 43 & 23 \\
\hline DUAL1 & 85 & 85 & 85 & 1 & 0 \\
\hline DUAL2 & 96 & 96 & 96 & 1 & 0 \\
\hline HIMMELBI & 100 & 100 & 100 & 0 & 12 \\
\hline DUAL3 & 111 & 111 & 111 & 1 & 0 \\
\hline SMBANK & 117 & 117 & 117 & 64 & 0 \\
\hline QPCBOEI2 & 143 & 143 & 54 & 4 & 181 \\
\hline QPNBOEI2 & 143 & 143 & 54 & 4 & 181 \\
\hline AGG & 163 & 163 & 0 & 36 & 452 \\
\hline HYDROELS & 167 & 167 & 167 & 0 & 4 \\
\hline GMNCASE1 & 175 & 0 & 0 & 0 & 300 \\
\hline GMNCASE4 & 175 & 0 & 0 & 0 & 350 \\
\hline GMNCASE2 & 175 & 0 & 0 & 0 & 1050 \\
\hline GMNCASE3 & 175 & 0 & 0 & 0 & 1050 \\
\hline SSEBLIN & 192 & 192 & 168 & 48 & 24 \\
\hline DALLASM & 196 & 196 & 196 & 151 & 0 \\
\hline PRIMALC1 & 230 & 215 & 0 & 0 & 1 \\
\hline PRIMALC2 & 231 & 229 & 0 & 0 & 1 \\
\hline PRIMALC5 & 287 & 278 & 0 & 0 & 1 \\
\hline PRIMAL1 & 325 & 1 & 0 & 0 & 85 \\
\hline QPCBOEI1 & 384 & 384 & 156 & 9 & 431 \\
\hline QPNBOEI1 & 384 & 384 & 156 & 9 & 431 \\
\hline QPCSTAIR & 385 & 379 & 6 & 209 & 147 \\
\hline QPNSTAIR & 385 & 379 & 6 & 209 & 147 \\
\hline STEENBRA & 432 & 432 & 0 & 108 & 0 \\
\hline STATIC3 & 434 & 144 & 0 & 96 & 0 \\
\hline STEENBRB & 468 & 468 & 0 & 108 & 0 \\
\hline STEENBRD & 468 & 468 & 0 & 108 & 0 \\
\hline STEENBRF & 468 & 468 & 0 & 108 & 0 \\
\hline
\end{tabular}



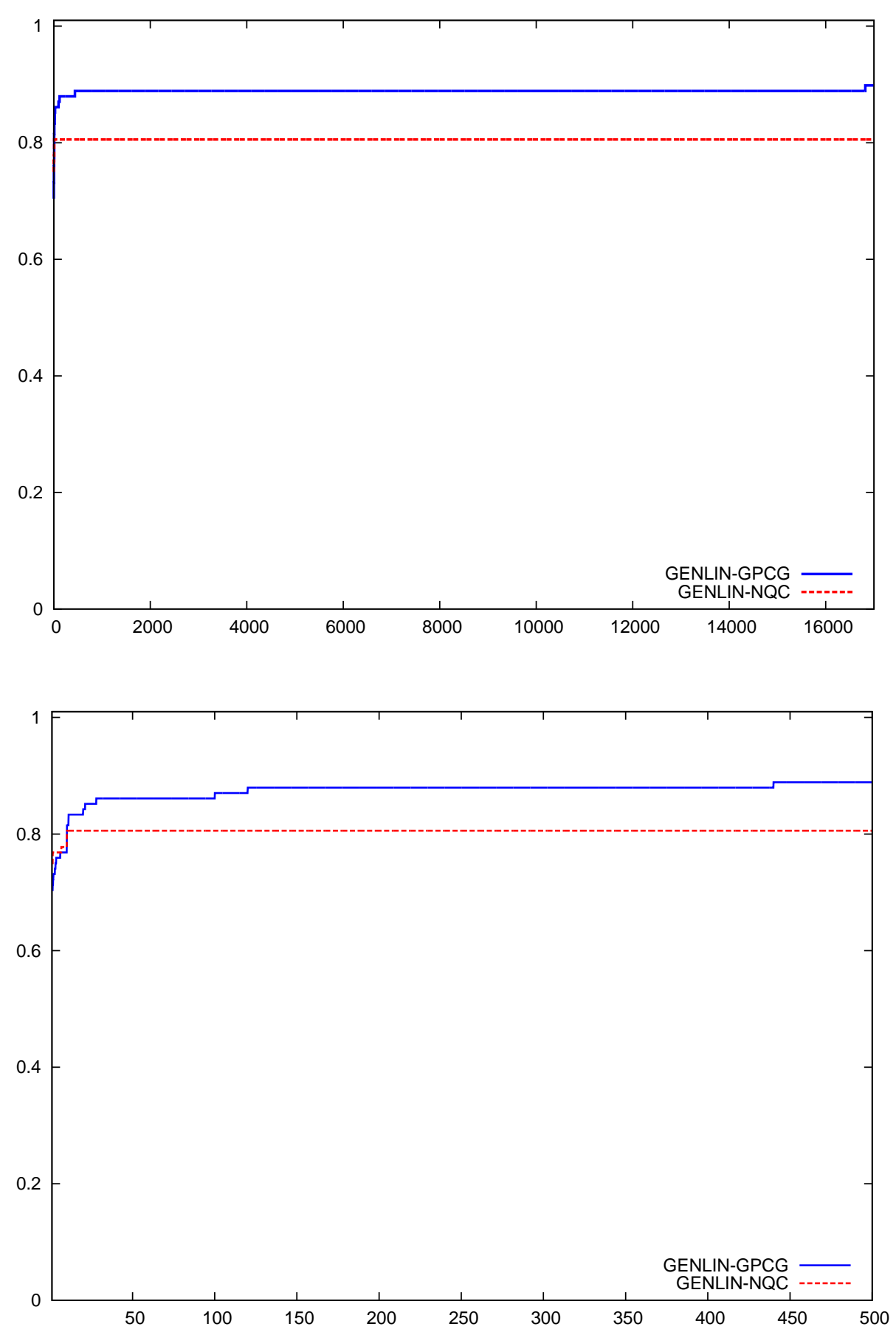

Figura 2.1: Curva de perfil de desempenho comparando GENLIN-GPCG com GENLIN-NQC usando tempo como medida de desempenho. O segundo gráfico é apenas um destaque da região mais à esquerda do primeiro gráfico. Para a construção destes gráficos, quando um dos dois métodos encontra valor de função diferente para um mesmo problema, consideramos que este método levou tempo infinito para encontrar a solução. 

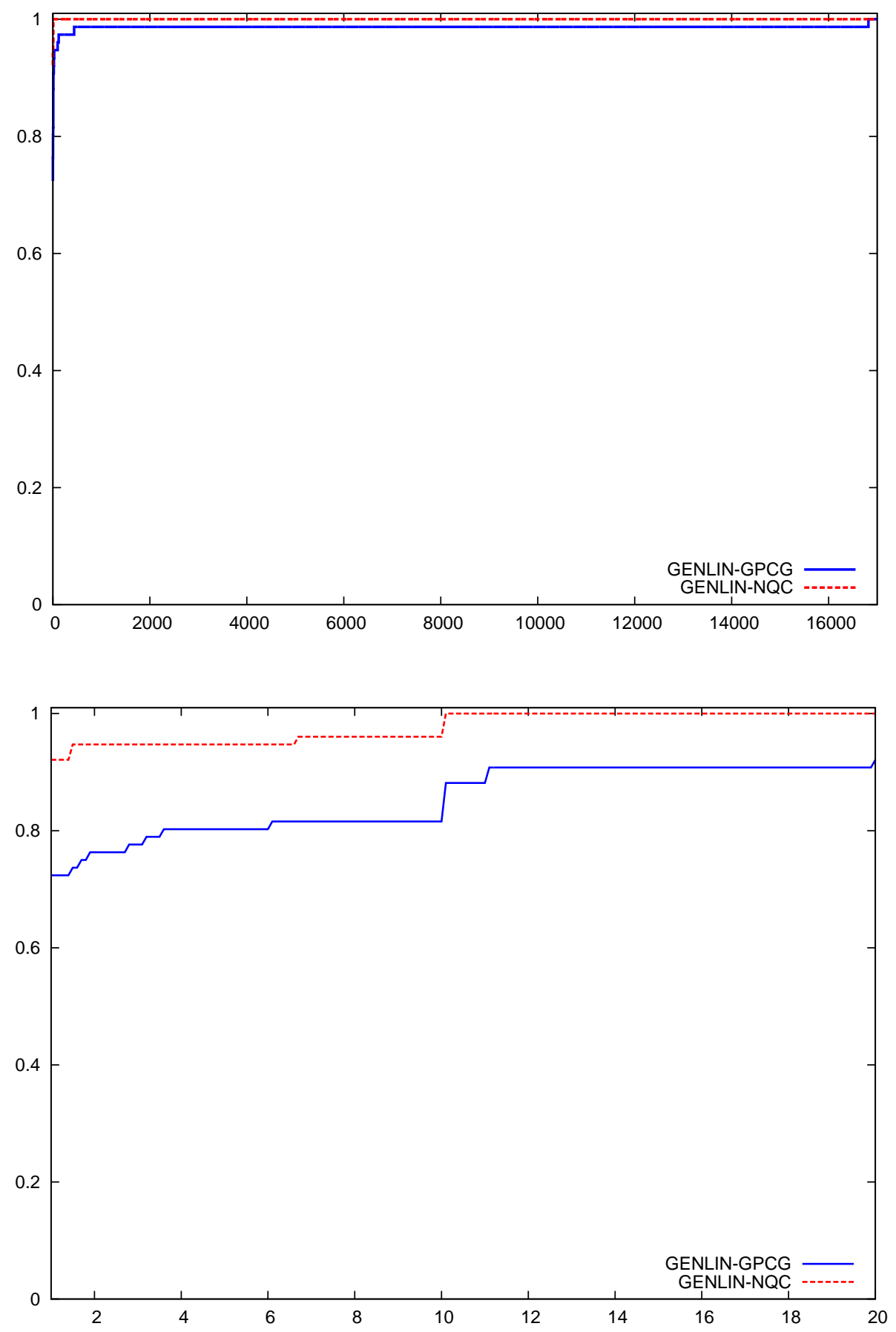

Figura 2.2: Curva de perfil de desempenho comparando GENLIN-GPCG com GENLIN-NQC usando tempo como medida de desempenho. O segundo gráfico é apenas um destaque da região mais à esquerda do primeiro gráfico. Para a construção destes gráficos, consideramos apenas os problemas para os quais ambos os métodos chegaram ao mesmo valor de função. 

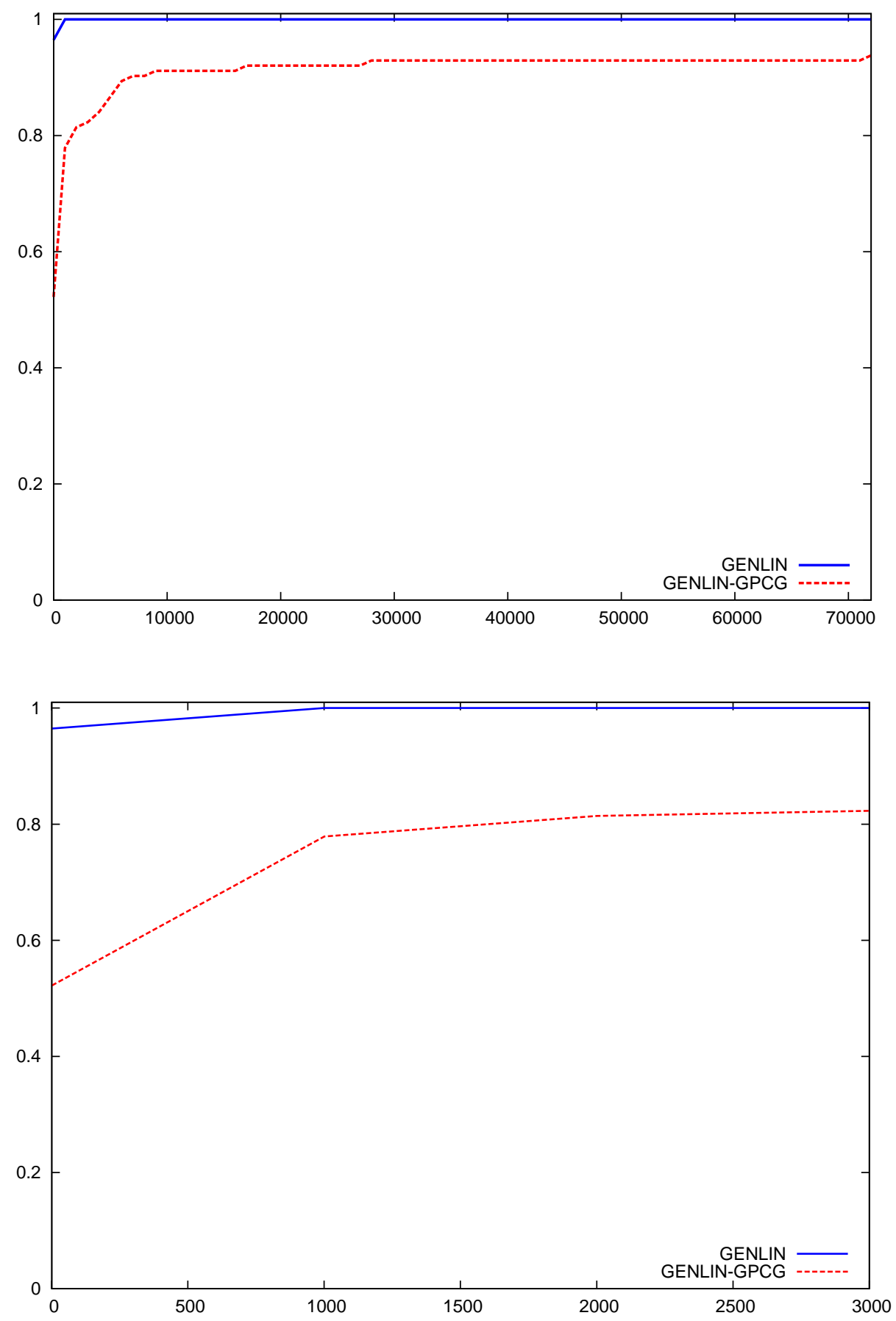

Figura 2.3: Curva de perfil de desempenho comparando GENLIN com GENLIN-GPCG usando tempo como medida de desempenho. O segundo gráfico é apenas um destaque da região mais à esquerda do primeiro gráfico. Para a construção destes gráficos, quando um dos dois métodos encontra valor de função diferente para um mesmo problema, consideramos que este método levou tempo infinito para encontrar a solução. 

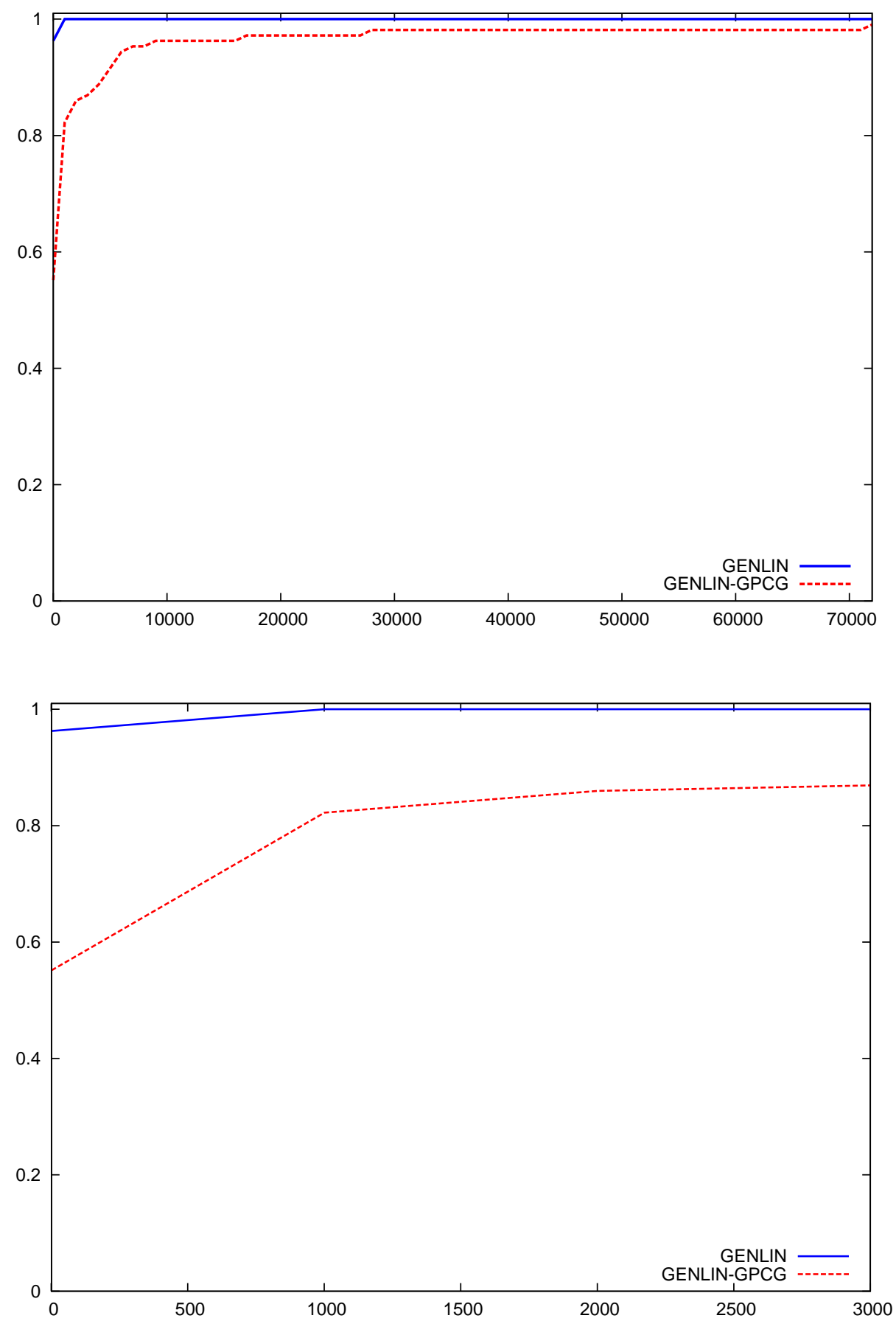

Figura 2.4: Curva de perfil de desempenho comparando GENLIN com GENLIN-GPCG usando tempo como medida de desempenho. O segundo gráfico é apenas um destaque da região mais à esquerda do primeiro gráfico. Para a construção destes gráficos, consideramos apenas os problemas para os quais ambos os métodos chegaram ao mesmo valor de função. 

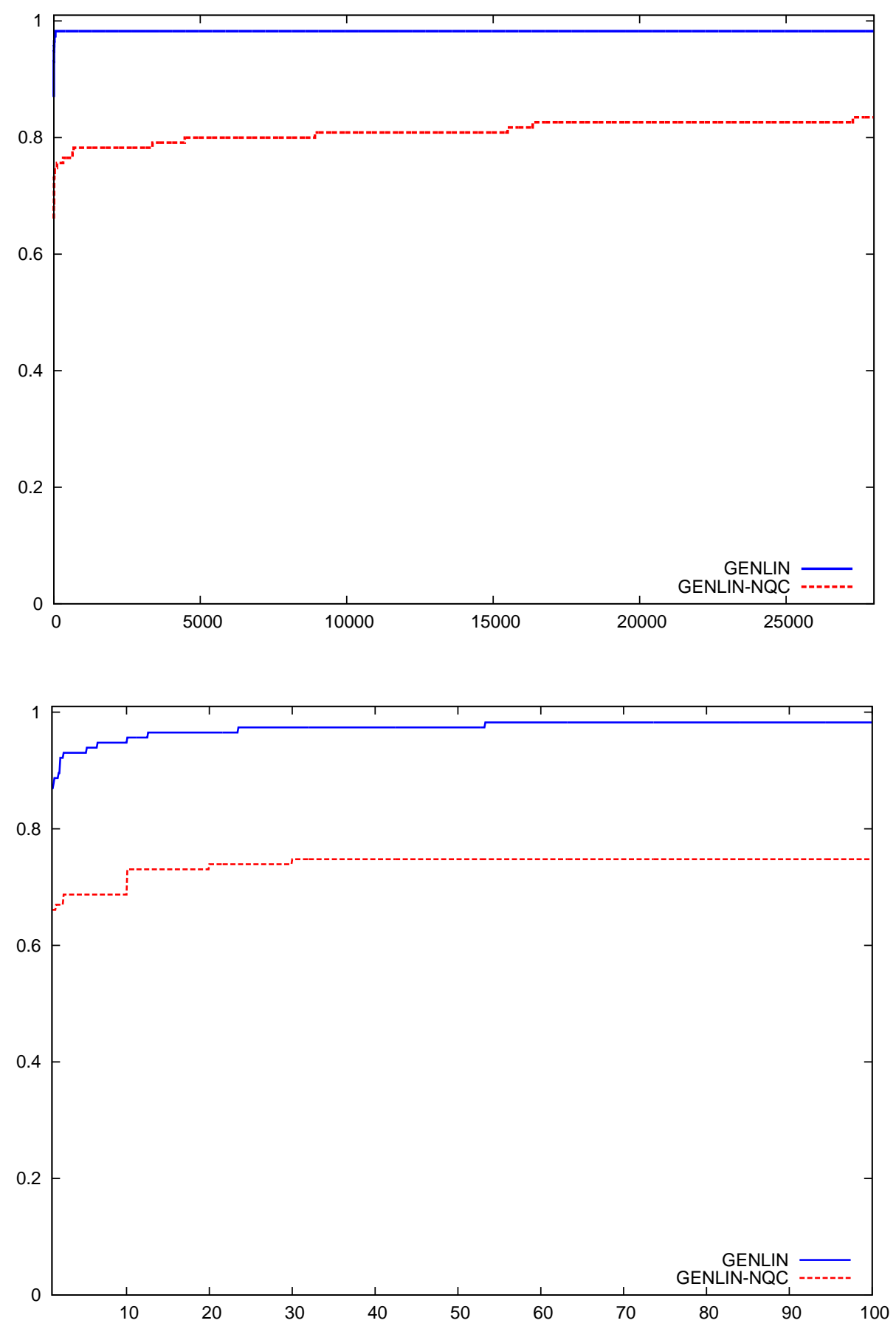

Figura 2.5: Curva de perfil de desempenho comparando GENLIN com GENLIN-NQC usando tempo como medida de desempenho. O segundo gráfico é apenas um destaque da região mais à esquerda do primeiro gráfico. Para a construção destes gráficos, quando um dos dois métodos encontra valor de função diferente para um mesmo problema, consideramos que este método levou tempo infinito para encontrar a solução. 

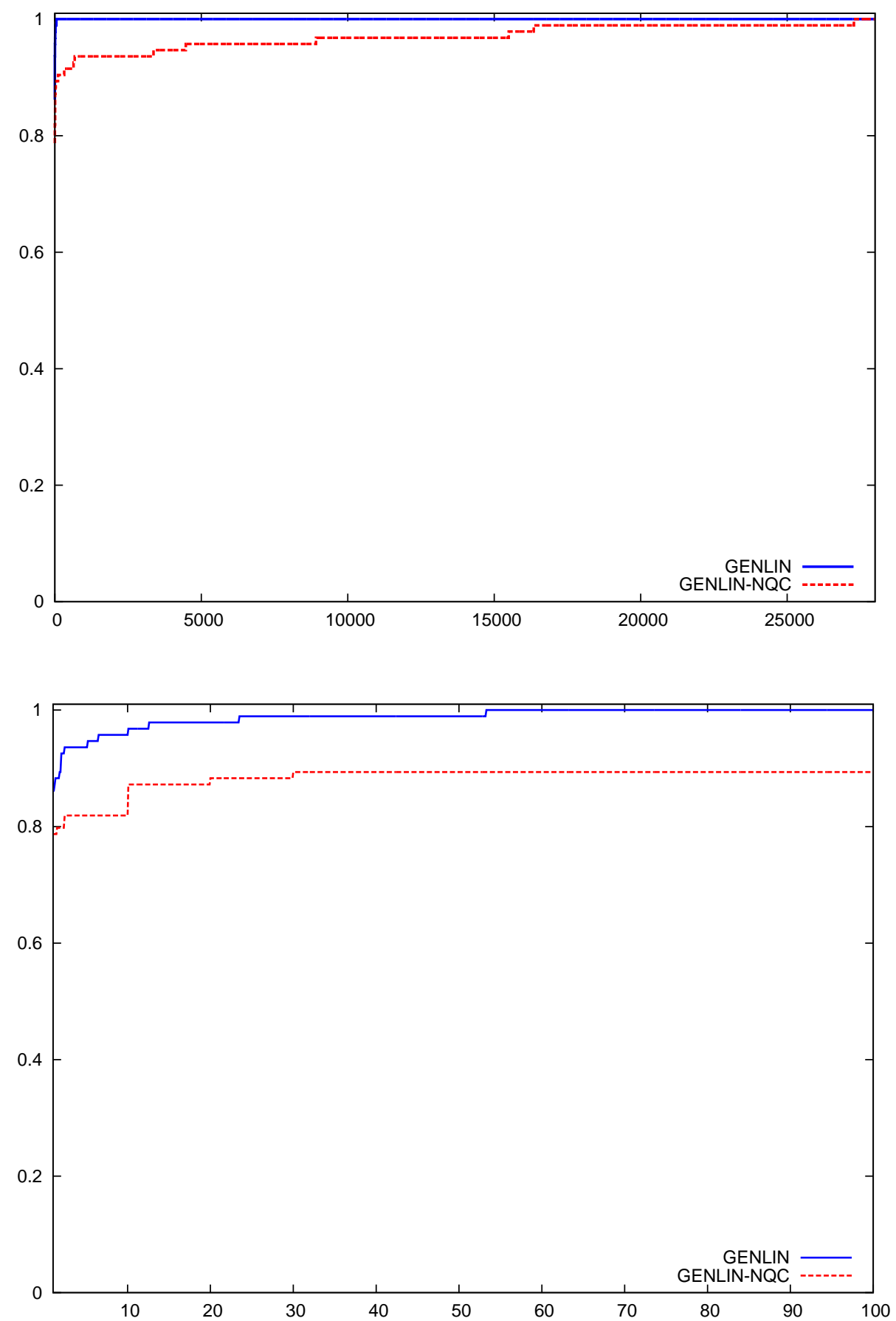

Figura 2.6: Curva de perfil de desempenho comparando GENLIN com GENLIN-NQC usando tempo como medida de desempenho. O segundo gráfico é apenas um destaque da região mais à esquerda do primeiro gráfico. Para a construção destes gráficos, consideramos apenas os problemas para os quais ambos os métodos chegaram ao mesmo valor de função. 


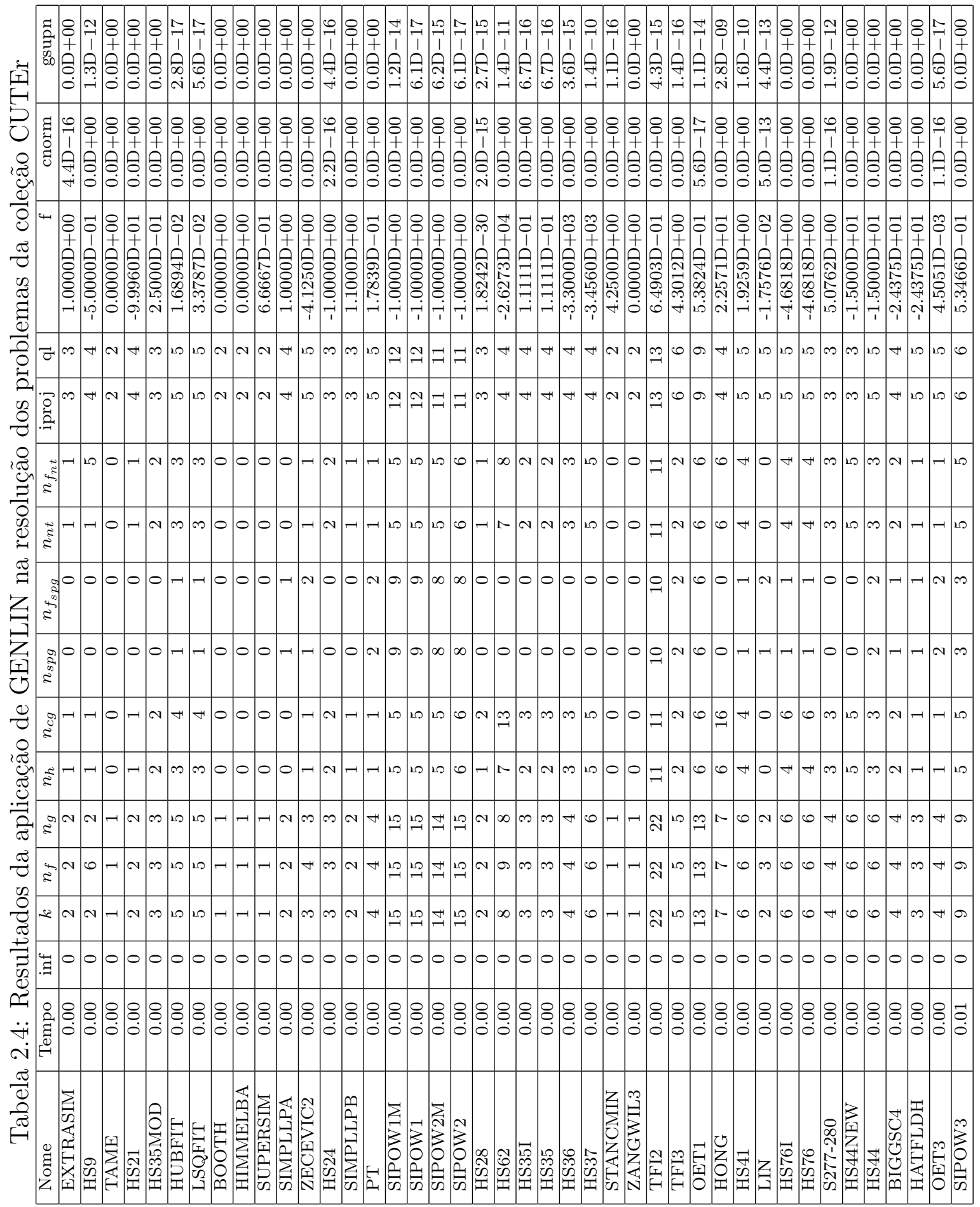




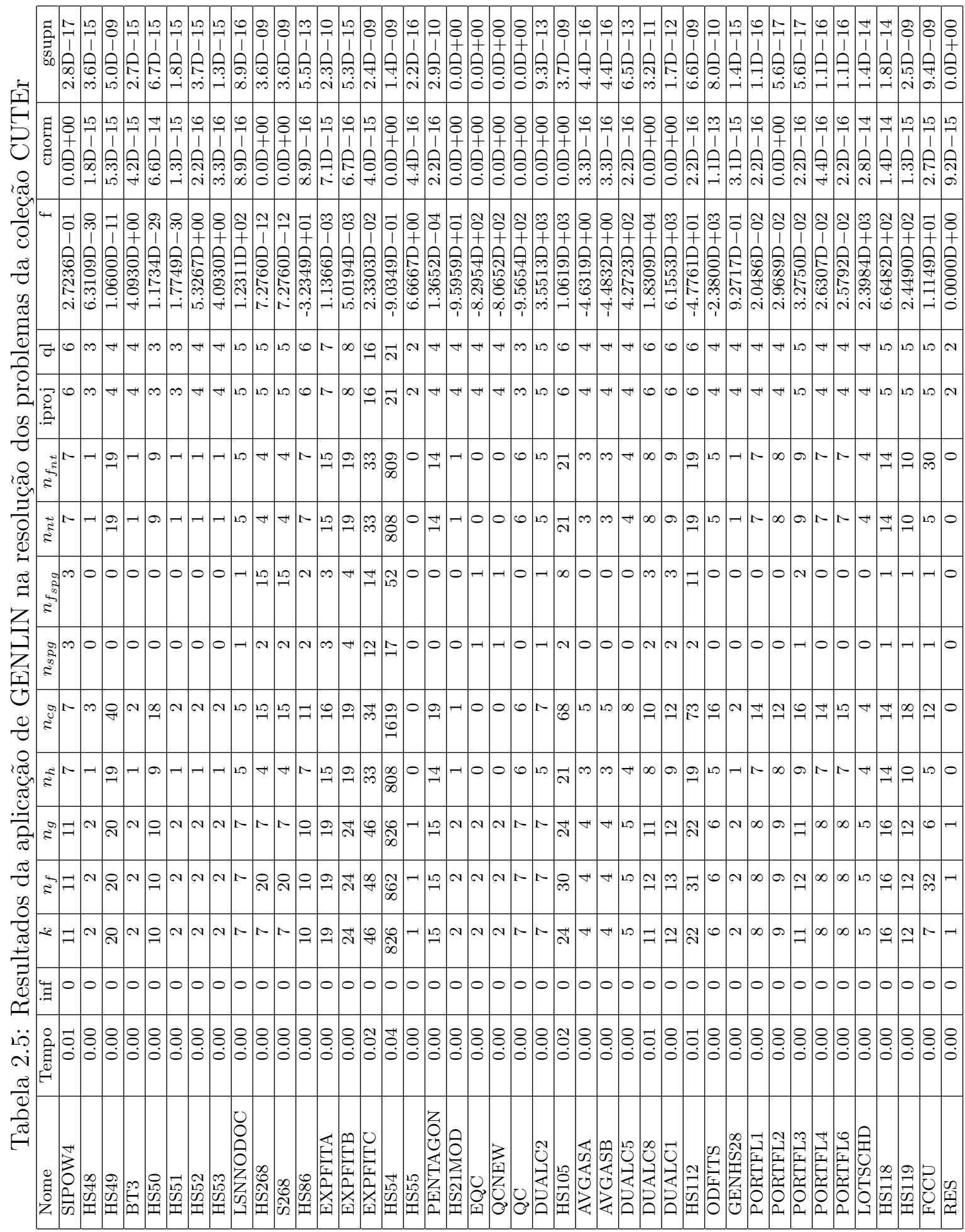




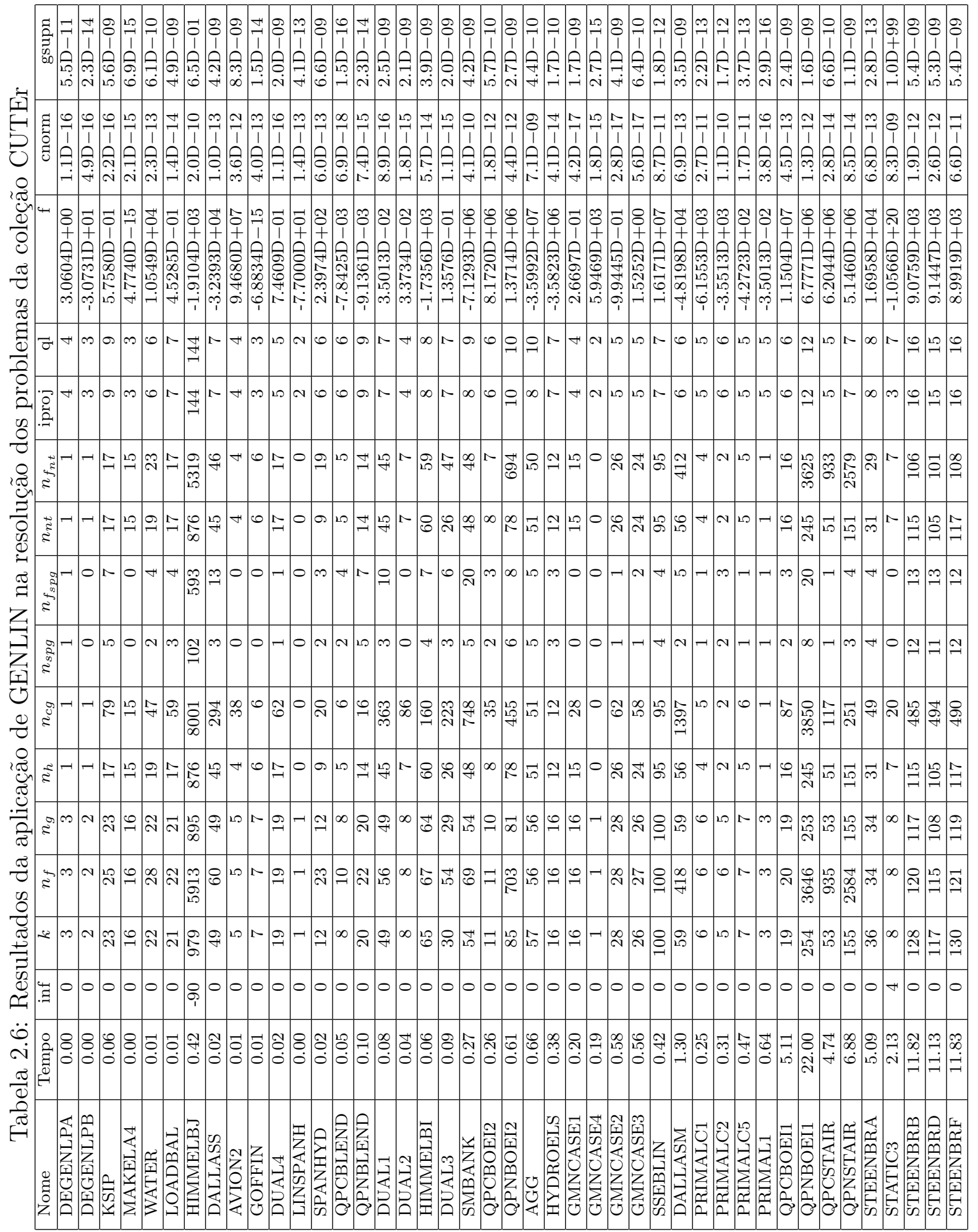




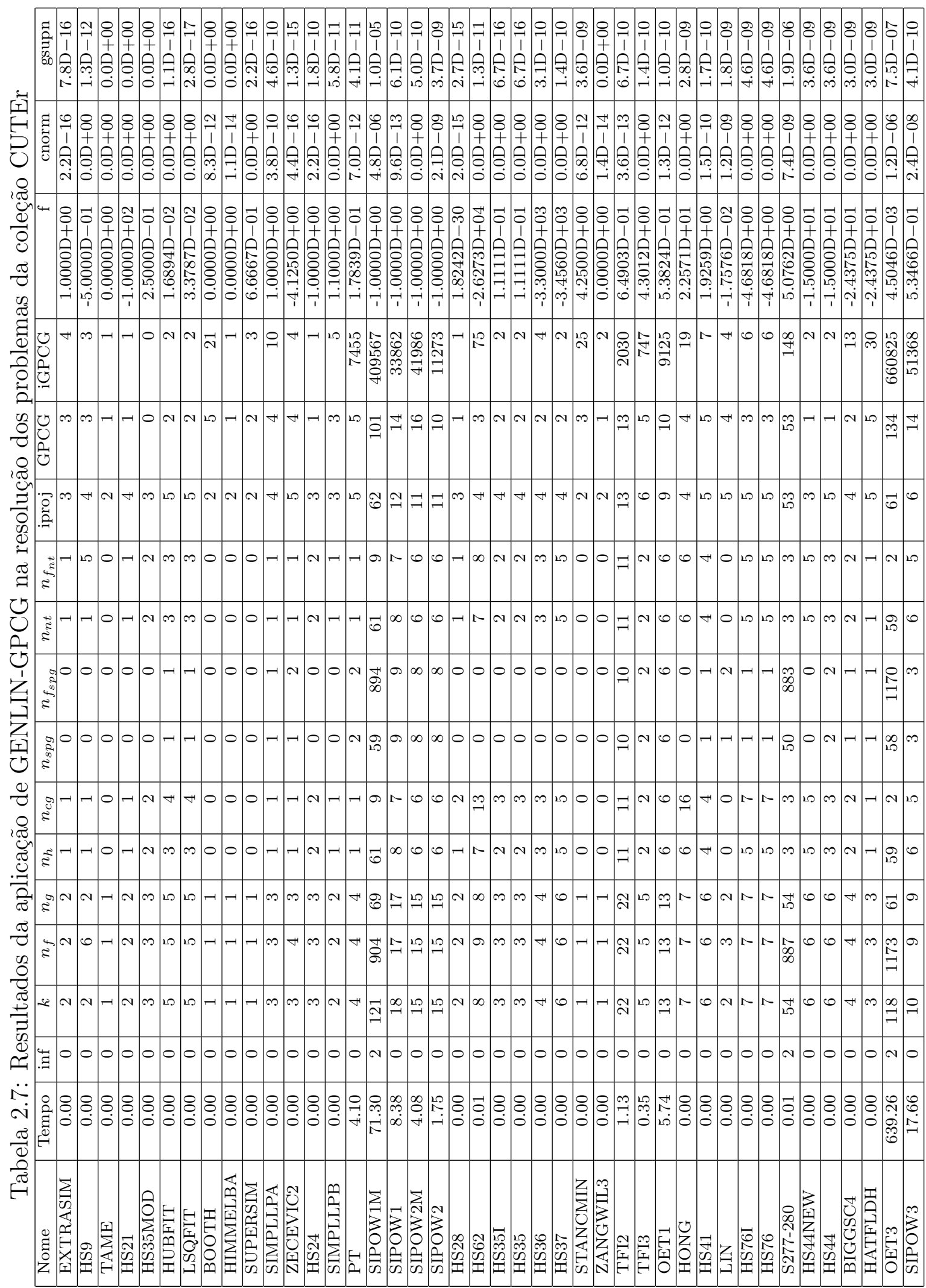




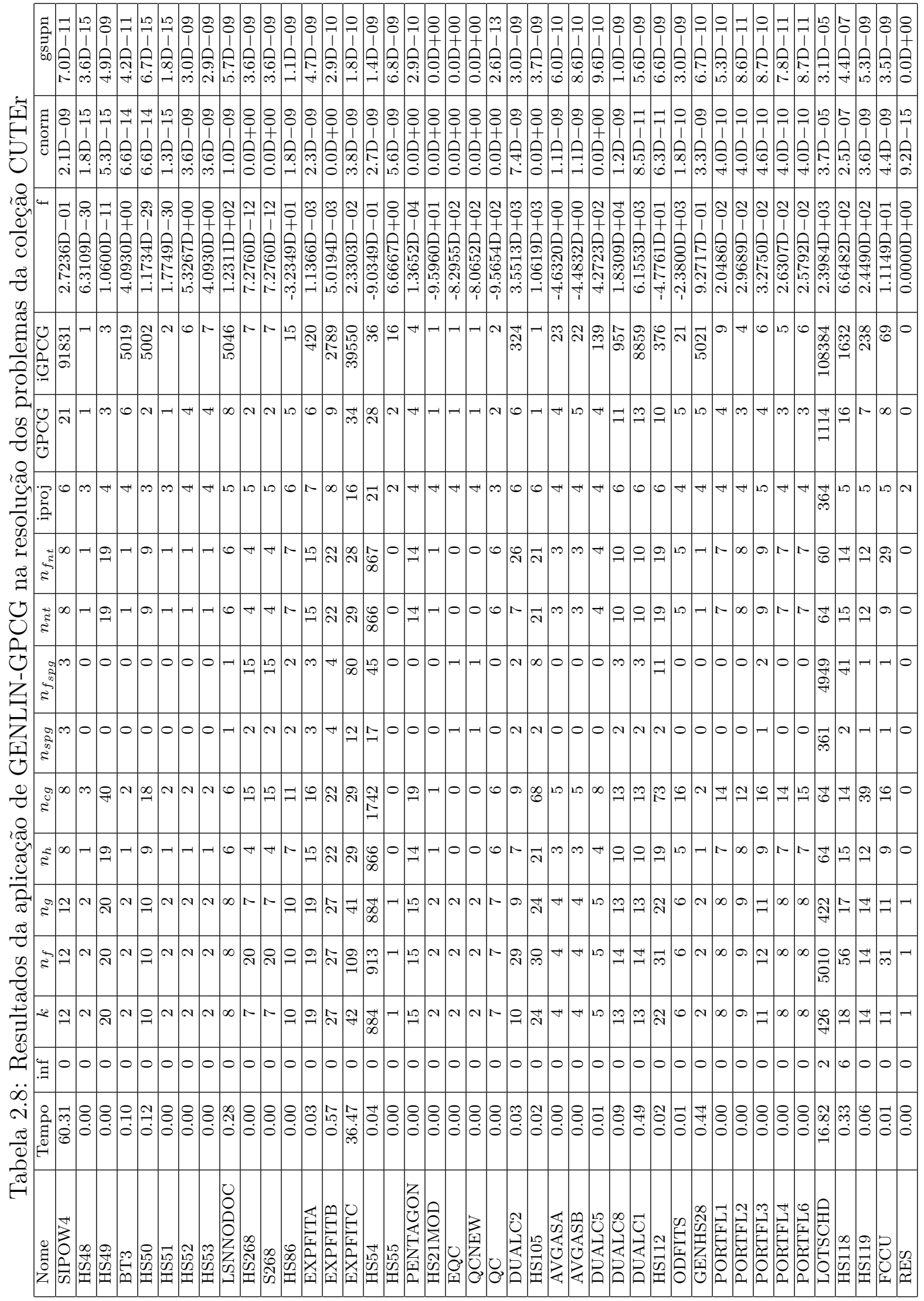




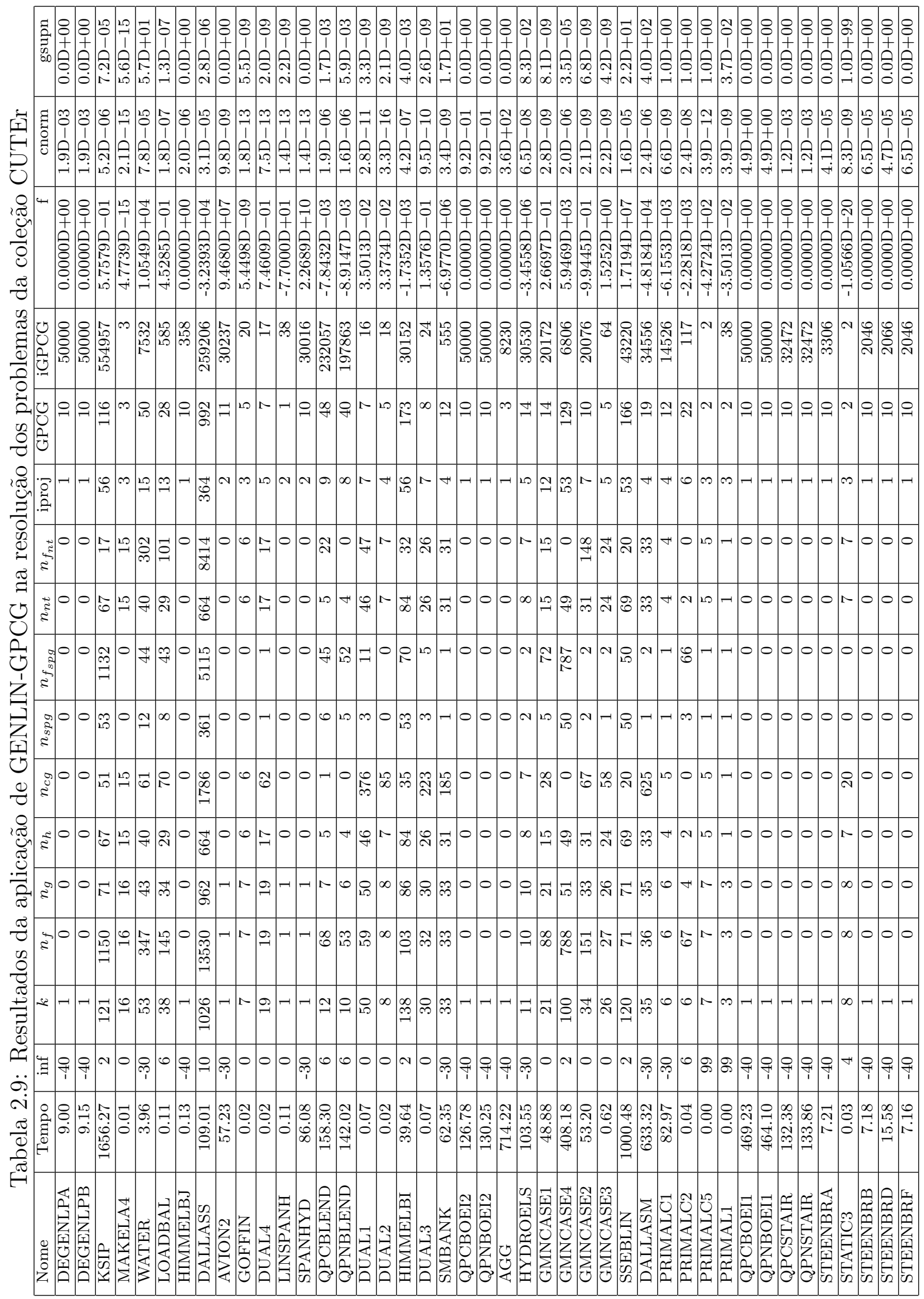




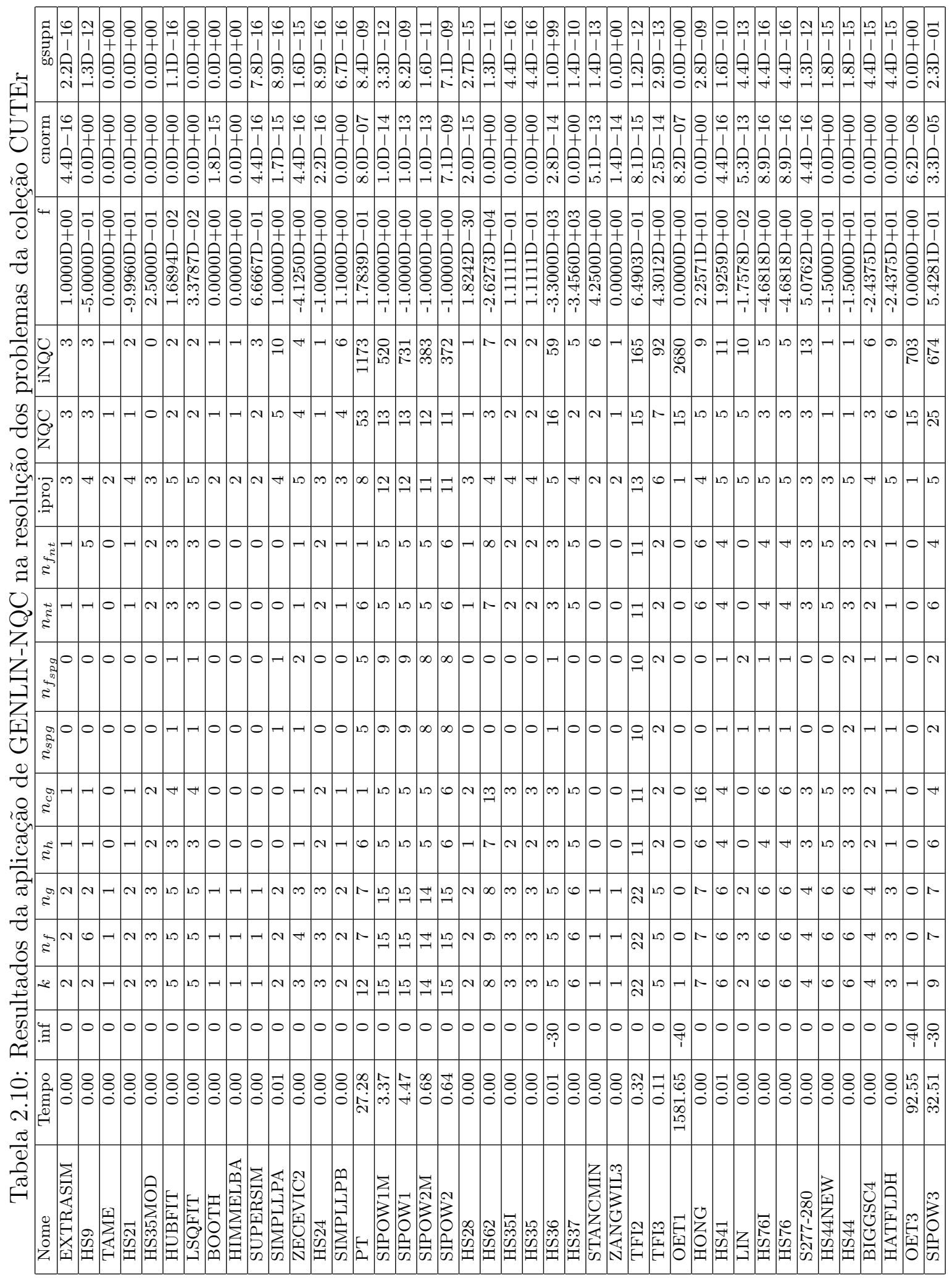




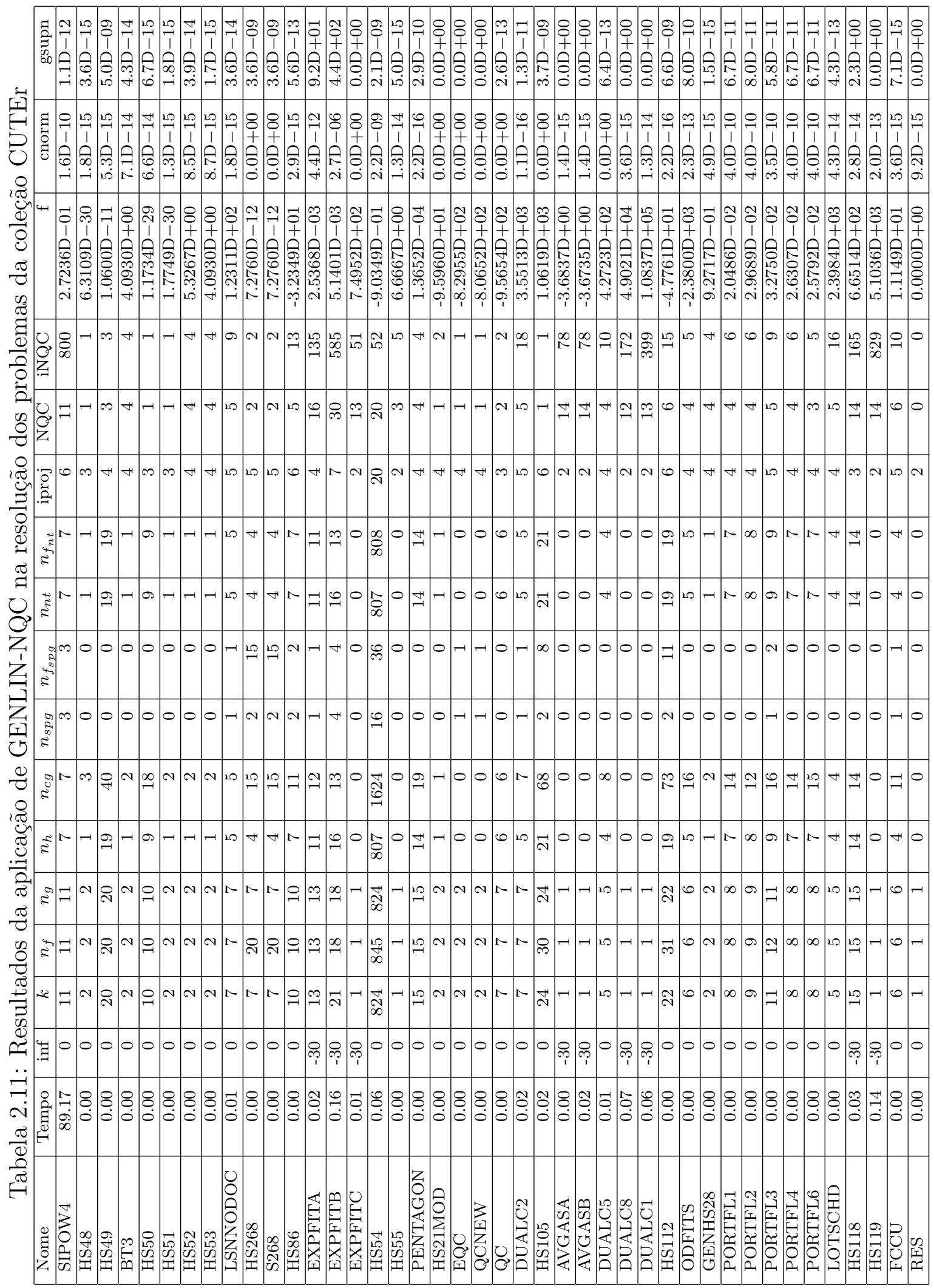




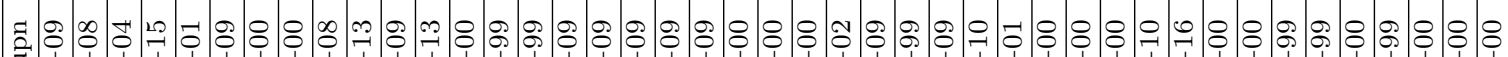
๘

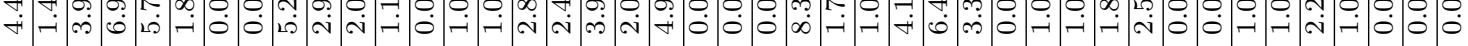

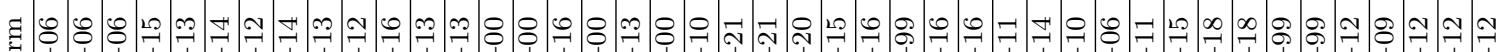

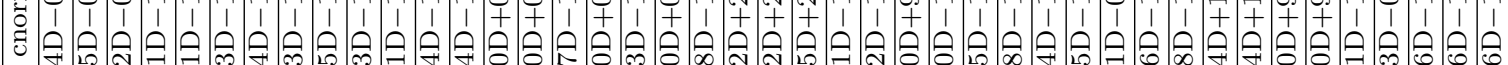

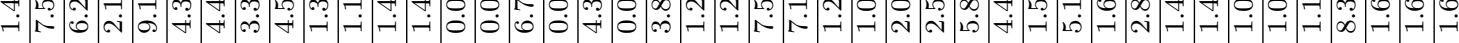

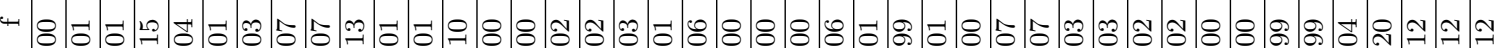

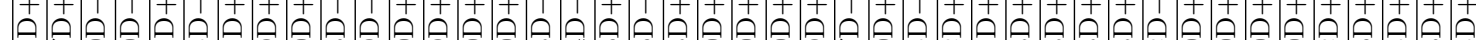

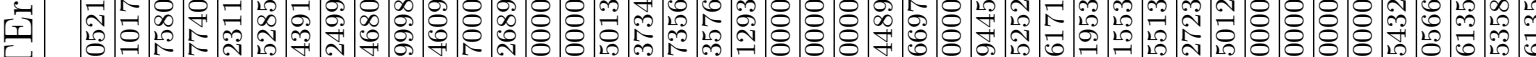

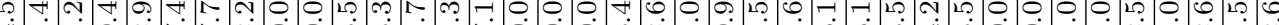

(2)

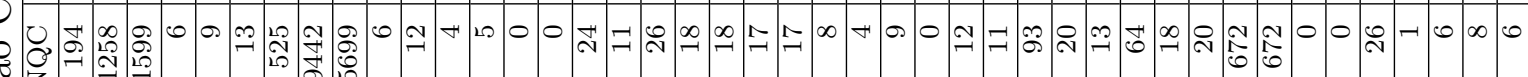

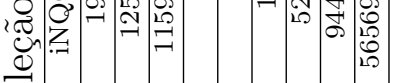

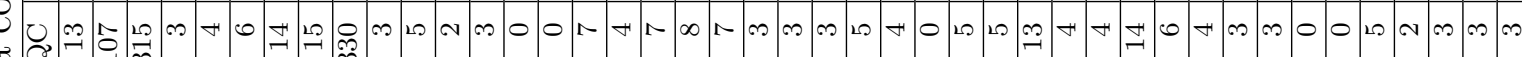
चु

శ్

za. $\frac{0}{0}$

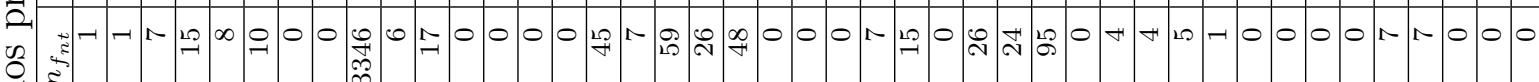
है हैं

,

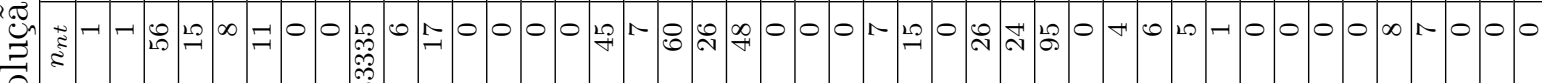
赵

ฮี O

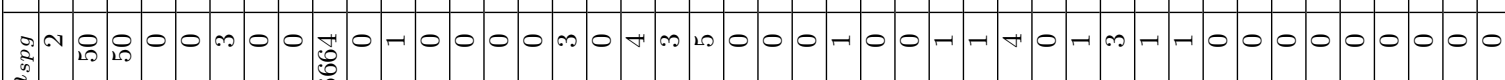
西

象 $\checkmark$

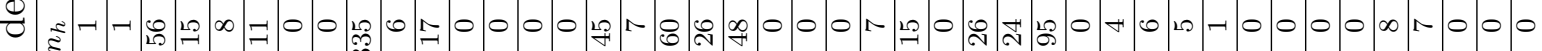
,

刍 党: శึ

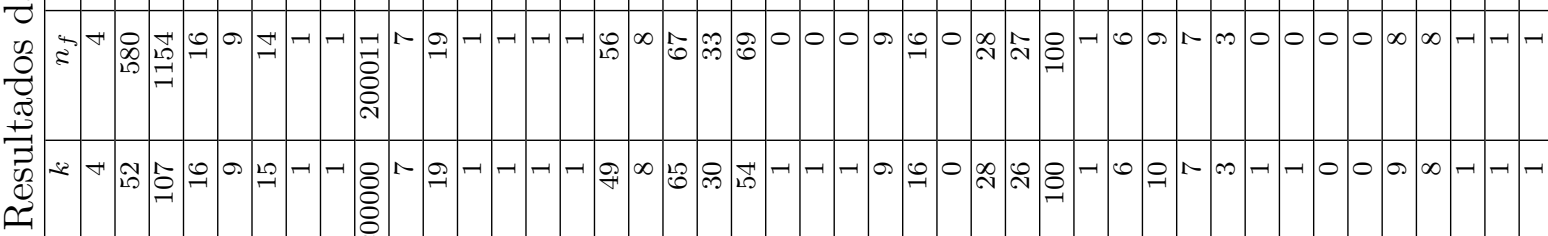

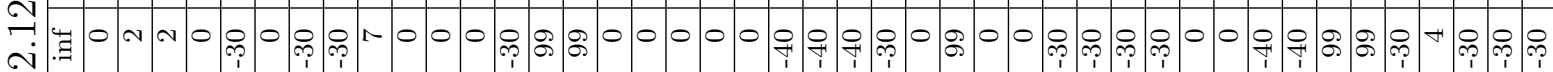

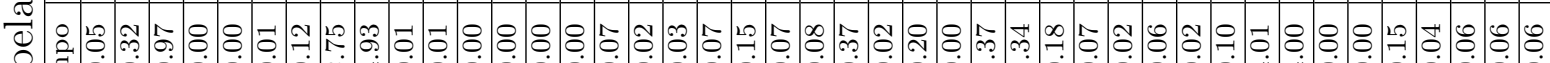
莡

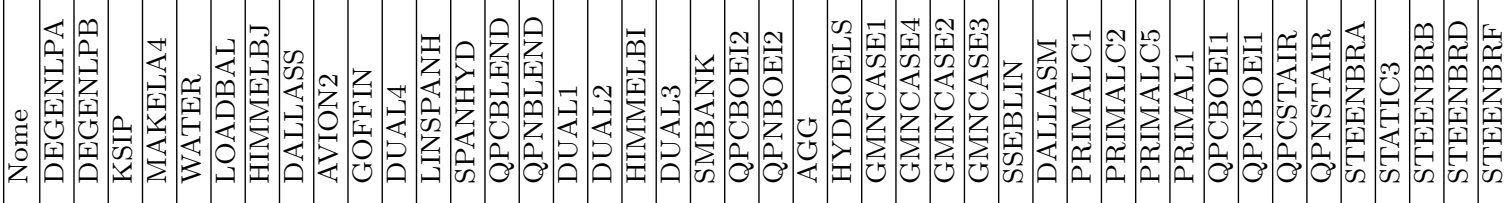




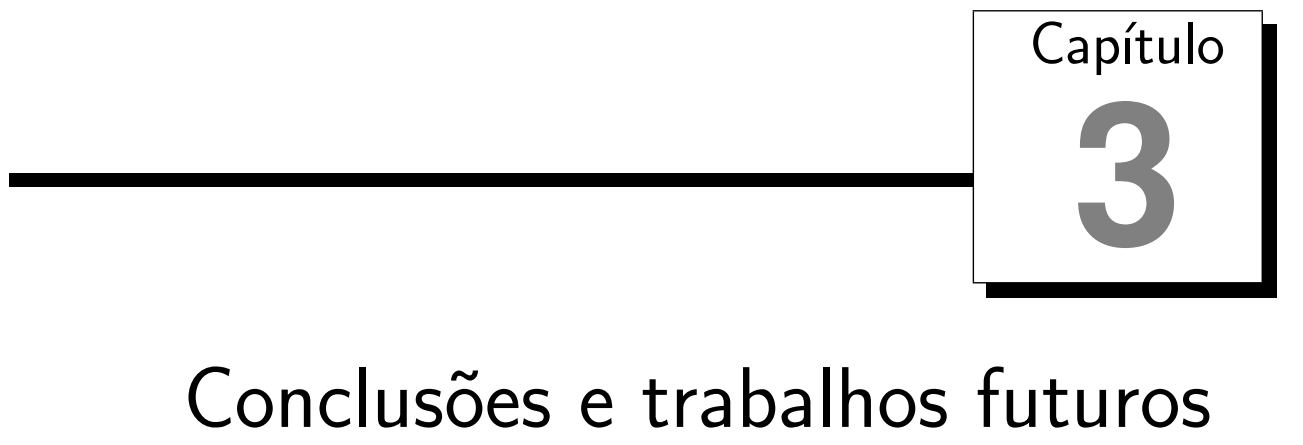

Neste trabalho, estudamos em detalhes o método Moré-Toraldo, apresentado em Moré e Toraldo (1991). O método Moré-Toraldo é um método clássico para resolução de problemas de programação quadrática convexa com restrições de caixa. A cada iteração, o método Moré-Toraldo usa o método de Gradientes Conjugados para explorar a face da região factível, e o método de Gradiente Projetado para mudar de face.

Implementamos, em Fortran 77, o método Moré-Toraldo, que chamamos GPCG. Reproduzimos os experimentos númericos feitos em Moré e Toraldo (1991), obtendo resultados semelhantes, atestando que a implementação GPCG está correta.

Estudamos o "método" NQC, um procedimento para resolução de problemas de programação quadrática convexa com restrições de caixa. O "método" NQC, desenvolvido e implementado pela orientadora Profa. Dra. Marina Andretta, durante seu pós-doutorado, em parceria com os professores José Mario Martínez (IMECC-Unicamp) e Mikhail Solodov (IMPA), usa o método do Gradiente Espectral Projetado para definir em que face trabalhar, e o método de Newton para calcular o minimizador da quadrática reduzida a esta face. Apesar de sua eficiência, este método não tem garantia de convergência. Chamamos de NQC à implementação deste procedimento. 
Usamos GPCG e NQC para resolver todos os problemas de programação quadrática com restrições de caixa e irrestritos da coleção CUTEr (Bongartz et al. (1995)).

As Hessianas dos problemas tomados da coleção CUTEr podem não ser definidas positivas. Nos experimentos numéricos de ambos os métodos, somamos um $\rho$ pequeno na diagonal da matriz Hessiana para tornar a função objetivo convexa.

O problema BQPGAUSS não foi levado em consideração já que GPCG parou por número máximo de iterações atingido e NQC parou ao encontrar um ciclo. Os problemas ODNAMUR e PALMER1D também não foram levados em consideração já que GPCG parou por número máximo de iterações atingidas e por progresso insuficiente, respectivamente. NQC satisfez o critério de convergência nestes dois problemas. Todos os problemas restante da coleção CUTEr foram resolvidos pelos métodos.

Ao comparar o desempenho de GPCG e NQC, NQC se mostrou um pouco mais robusto que o GPCG. Por outro lado, GPCG se mostrou um pouco mais eficiente.

Neste trabalho, também estudamos GENLIN, apresentado em Andretta et al. (2010). GENLIN é a implementação de um método de restrições ativas para resolução de problemas de minimização com restrições lineares de pequeno e médio porte, que faz parte do Projeto TANGO (Trustable Algorithms for Nonlinear General Optimization) ${ }^{1}$.

Tanto GPCG como NQC foram inclusos em GENLIN para calcular projeções de maneira esparsa, usando uma formulação dual. Desse modo, obtivemos dois novos métodos com projeção esparsa, GENLIN-GPCG e GENLIN-NQC, os quais usam as rotinas GPCG e NQC, respectivamente. Utilizamos os problemas da coleção CUTEr que possuem um número limitado de variáveis e apenas restrições lineares e de caixa para verificar o desempenho destes dois novos métodos, isso porque, apesar de a projeção ser esparsa para estas novas versões de GENLIN, outras partes essenciais de GENLIN usam álgebra linear

\footnotetext{
${ }^{1}$ Disponível em www.ime.usp.br $/{ }^{\sim}$ egbirgin/tango.
} 
densa.

Como o dual do problema de projeção é muito mal condicionado, infelizmente os resultados não foram muito animadores, mostrando que a versão original de GENLIN é mais robusta e mais eficiente.

Algumas sugestões para trabalhos futuros são:

- Estudar outros métodos para programação quadrática convexa, talvez tentando resolver o problema primal de forma esparsa, evitando, assim, o mau-condicionamento do problema.

- Com relação a GENLIN, para que tenhamos uma versão esparsa, é necessário que seja mudado o modo como é feito o cálculo da matriz base do núcleo $Z$ (usando redução de variáveis, por exemplo). Com isso, poderão ser testados problemas maiores. E uma alternativa ao uso de métodos de programação quadrática para resolver o problema de projeção seria eliminar as projeções. Isso geraria um método essencialmente diferente do GENLIN atual, que é adequado para a resolução de problemas de pequeno e médio porte. 
Apêndice

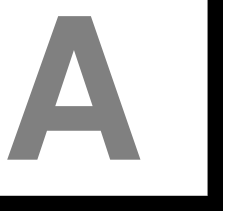

\section{Provas de teoremas, proposições e propriedades}

Teorema 1.1. O problema de programação quadrática com restrições de caixa (1.1) tem solução única $x^{*}$ quando q é estritamente convexa na região factível (1.2).

Prova: Como $q$ é convexa em $\Omega_{\square}$, o problema (1.1) possui ao menos uma solução. Admita duas soluções diferentes para o problema (1.1) $x_{1}, x_{2}$, tais que $q\left(x_{1}\right)=q\left(x_{2}\right)$.

Seja $\bar{x}=\lambda x_{1}+(1-\lambda) x_{2}$, com $\lambda \in(0,1)$. Como $x_{1}, x_{2} \in \Omega_{\square}$, então $\bar{x} \in \Omega_{\square}$ e assim, devido a $q$ ser estritamente convexa,

$$
q(\bar{x})=q\left(\lambda x_{1}+(1-\lambda) x_{2}\right)<\lambda q\left(x_{1}\right)+(1-\lambda) q\left(x_{2}\right) .
$$

Como $q\left(x_{1}\right)=q\left(x_{2}\right)$, temos que $q(\bar{x})<q\left(x_{2}\right)$, concluindo que $x_{2}$ não é mínimo.

Fica mostrado que o problema (1.1) tem solução única.

Teorema 1.2. Considere $q$ uma função convexa sobre o conjunto $\Omega_{\square} . O$ 
ponto $x^{*}$ é minimizador da função q sobre o conjunto $\Omega_{\square}$ se, e somente se, $\nabla q\left(x^{*}\right)^{T}\left(x-x^{*}\right) \geq 0$, para todo $x \in \Omega_{\square}$.

Prova: Suponha que $x^{*}$ é minimizador da função $q$ sobre o conjunto $\Omega_{\square} \mathrm{e}$ que $\nabla q\left(x^{*}\right)^{T}\left(x-x^{*}\right)<0$ para algum $x \in \Omega_{\square}$.

Para $\epsilon>0$, defina $g(\epsilon)=q\left(x^{*}+\epsilon\left(x-x^{*}\right)\right)$. Assim, pelo teorema do Valor Médio, temos que existe $s \in[0,1]$, tal que

$$
g(\epsilon)-g(0)=g^{\prime}(s \epsilon) \epsilon
$$

Ou seja,

$$
q\left(x^{*}+\epsilon\left(x-x^{*}\right)\right)=q\left(x^{*}\right)+\epsilon \nabla q\left(x^{*}+s \epsilon\left(x-x^{*}\right)\right)^{T}\left(x-x^{*}\right) .
$$

Como $\nabla q$ é contínua, para $\epsilon$ suficientemente pequeno, $\nabla q\left(x^{*}+s \epsilon(x-\right.$ $\left.\left.x^{*}\right)\right)^{T}\left(x-x^{*}\right)<0$. Assim, $q\left(x^{*}+\epsilon\left(x-x^{*}\right)\right)<q\left(x^{*}\right)$, o que é absurdo, já que $x^{*}$ é minimizador (note que o vetor $x^{*}+\epsilon\left(x-x^{*}\right.$ ) é factível para todo $\epsilon \in[0,1]$, devido a $\Omega_{\square}$ ser convexo).

Portanto, se $x^{*}$ é minimizador de $q$ sobre o conjunto $\Omega_{\square}$, então $\nabla q\left(x^{*}\right)^{T}(x-$ $\left.x^{*}\right) \geq 0$, para todo $x \in \Omega_{\square}$.

Agora, como $q$ é uma função convexa sobre o conjunto $\Omega_{\square}$, temos que

$$
q(x) \geq q\left(x^{*}\right)+\nabla q\left(x^{*}\right)^{T}\left(x-x^{*}\right), \quad \forall x \in \Omega_{\square} .
$$

Portanto, se $\nabla q\left(x^{*}\right)^{T}\left(x-x^{*}\right) \geq 0$, concluímos que $q(x) \geq q\left(x^{*}\right)$ para todo $x \in \Omega_{\square}$, assim $x^{*}$ é minimizador de $q$ sobre o conjunto $\Omega_{\square}$.

Proposição 1.1. Um ponto $x^{*}$ viável satisfaz $P_{\Omega_{\square}}\left(x^{*}-\nabla q\left(x^{*}\right)\right)-x^{*}=0$, se, e somente se, este ponto também satisfaz as condições KKT (1.4).

Prova: Se um ponto $x^{*}$ satisfaz as condições KKT (1.4), deduzimos que:

- Se $\left[x^{*}\right]_{i} \in\left([l]_{i},[u]_{i}\right)$, então $\left[\lambda^{*}\right]_{i}=\left[\mu^{*}\right]_{i}=0$. Assim, $\left[\nabla q\left(x^{*}\right)\right]_{i}=0 \mathrm{e}$ $P_{\Omega_{\square}}\left(\left[x^{*}\right]_{i}\right)-[x]_{i}^{*}=0$. 
- Se $\left[x^{*}\right]_{i}=[l]_{i}$, então $\left[\lambda^{*}\right]_{i} \geq 0$ e $\left[\mu^{*}\right]_{i}=0$. Assim, $\left[\nabla q\left(x^{*}\right)\right]_{i} \geq 0$ e $\left[x^{*}-\nabla q\left(x^{*}\right)\right]_{i}=\left[l^{*}-\nabla q\left(x^{*}\right)\right]_{i} \leq[l]_{i}$. Ou seja, $P_{\Omega_{\square}}\left(\left[x^{*}-\nabla q\left(x^{*}\right)\right]_{i}\right)-[x]_{i}^{*}=$ 0 .

- Se $\left[x^{*}\right]_{i}=[u]_{i}$, então $\left[\lambda^{*}\right]_{i}=0$ e $\left[\mu^{*}\right]_{i} \geq 0$. Assim, $\left[\nabla q\left(x^{*}\right)\right]_{i} \leq 0$ e $\left[x^{*}-\right.$ $\left.\nabla q\left(x^{*}\right)\right]_{i}=\left[u^{*}-\nabla q\left(x^{*}\right)\right]_{i} \geq[u]_{i}$. Ou seja, $P_{\Omega_{\square}}\left(\left[x^{*}-\nabla q\left(x^{*}\right)\right]_{i}\right)-[x]_{i}^{*}=0$

Portanto, se um ponto $x^{*}$ satisfaz as condições KKT (1.4), então $P_{\Omega_{\square}}\left(x^{*}-\right.$ $\left.\nabla q\left(x^{*}\right)\right)-x^{*}=0$.

Agora, se um ponto $x^{*}$ viável satisfaz $P_{\Omega_{\square}}\left(x^{*}-\nabla q\left(x^{*}\right)\right)-x^{*}=0$, ou seja, satisfaz $\left[P_{\Omega_{\square}}\left(x^{*}-\nabla q\left(x^{*}\right)\right)\right]_{i}=\left[x^{*}\right]_{i}$, podemos deduzir que:

- Se $\left[x^{*}\right]_{i} \in\left([l]_{i},[u]_{i}\right)$, então $\left[\nabla q\left(x^{*}\right)\right]_{i}=0$.

- Se $\left[x^{*}\right]_{i}=[l]_{i}$, então $\left[\nabla q\left(x^{*}\right)\right]_{i} \geq 0$.

- Se $\left[x^{*}\right]_{i}=[u]_{i}$, então $\left[\nabla q\left(x^{*}\right)\right]_{i} \leq 0$.

Portanto, se um ponto $x^{*}$ viável satisfaz $P_{\Omega_{\square}}\left(x^{*}-\nabla q\left(x^{*}\right)\right)-x^{*}=0$, satisfaz as condições (1.5), que é uma simplificação das condições KKT (1.4).

Proposição 1.2. Um ponto $x^{*}$ viável satisfaz $\nabla_{\Omega_{\square}} q\left(x^{*}\right)=0$, se, e somente se, este ponto também satisfaz as condições KKT (1.4).

Prova: Se um ponto $x^{*}$ viável satisfaz $\nabla_{\Omega_{\square}} q\left(x^{*}\right)=0$, pela Definição 1.3, deduzimos que:

- Se $\left[x^{*}\right]_{i} \in\left([l]_{i},[u]_{i}\right)$, então $\left[\nabla q\left(x^{*}\right)\right]_{i}=0$.

- Se $\left[x^{*}\right]_{i}=[l]_{i}$, então $\left[\nabla q\left(x^{*}\right)\right]_{i} \geq 0$.

- Se $\left[x^{*}\right]_{i}=[u]_{i}$, então $\left[\nabla q\left(x^{*}\right)\right]_{i} \leq 0$.

Portanto, se um ponto $x^{*}$ viável satisfaz $\nabla_{\Omega_{\square}} q\left(x^{*}\right)=0$, satisfaz as condições (1.5), que é uma simplificação das condições KKT (1.4).

A prova de que se um ponto $x^{*}$ viável satisfaz as condições KKT (1.4), então este ponto também satisfaz $\nabla_{\Omega_{\square}} q\left(x^{*}\right)=0$, é análoga. 
Proposição 1.3. Considere $x^{*}$ o minimizador do problema (1.1). Se $x$ está suficientemente perto de $x^{*}$ e $x$ pertence à mesma face que $x^{*}$, então $x$ é um minimizador aproximado do problema (1.1).

Prova: Como $x$ pertence à mesma face que $x^{*}, x$ está suficientemente perto de $x^{*}$ e $q$ é uma função contínua, afirmamos, pelas condições (1.5), que:

- Se $\left[x^{*}\right]_{i}=[x]_{i}=[l]_{i}$, então $[\nabla q(x)]_{i} \geq-\epsilon_{i}$.

- Se $\left[x^{*}\right]_{i}=[x]_{i}=[u]_{i}$, então $[\nabla q(x)]_{i} \leq \epsilon_{i}$.

- Se $[x]_{i} \in\left([l]_{i},[u]_{i}\right)$, então $\left|[\nabla q(x)]_{i}\right|<\epsilon_{i}$,

sendo $\epsilon_{i}>0$ pequeno, $1 \leq i \leq n$. Portanto, $x$ satisfaz (1.7) e (1.8), ou seja, $x$ é um minimizador aproximado do problema (1.1).

Propriedade 1.2. Seja $k$ a iteração atual e $\phi_{k}: \mathbb{R} \rightarrow \mathbb{R}$ uma função definida por $\phi_{k}(\alpha)=q\left(P_{\Omega_{\square}}\left(x_{k}+\alpha d_{k}\right)\right)$. Então

$$
\alpha_{k}^{(0)}=-\frac{\phi_{k}^{\prime}(0)}{\phi_{k}^{\prime \prime}(0)}
$$

Se $d_{k}=-\nabla q\left(x_{k}\right)$, então

$$
\alpha_{k}^{(0)}=\frac{\left\|r_{k}\right\|_{2}^{2}}{r_{k}^{T} H r_{k}}
$$

e, no caso $d_{k}=Z_{k} w_{j_{k}}$, temos que

$$
\alpha_{k}^{(0)}=-\frac{r_{k}^{T} w_{j_{k}}}{\left(w_{j_{k}}\right)^{T} H_{k} w_{j_{k}}}=1
$$

Prova: Como o valor inicial testado $\alpha_{k}^{(0)}$ é o minimizador da função quadrática convexa que representa $\phi_{k}$ em $\left[0, \beta_{1}\right]$, vamos derivar $\phi_{k}$ e igualar a zero para encontrar o minimizador.

Lembrando que $\phi_{k}(\alpha)=q\left(P_{\Omega_{\square}}\left(x_{k}+\alpha d_{k}\right)\right)$ e, como $\alpha \in\left[0, \beta_{1}\right]$, então $x_{k}+\alpha d_{k}$ 
é factível. Ou seja $\phi_{k}(\alpha)=q\left(x_{k}+\alpha d_{k}\right)$. Assim,

$$
\begin{aligned}
\phi_{k}^{\prime}(\alpha) & =\nabla q\left(x_{k}+\alpha d_{k}\right)^{T} d_{k} \\
& =\left(H\left(x_{k}+\alpha d_{k}\right)+c\right)^{T} d_{k} \\
& =x_{k}^{T} H d_{k}+\alpha d_{k}^{T} H d_{k}+c^{T} d_{k} .
\end{aligned}
$$

Igualando a zero, temos que

$$
\alpha=\alpha_{k}^{(0)}=-\frac{\left(H x_{k}+c\right)^{T} d_{k}}{d_{k}^{T} H d_{k}}=-\frac{\phi_{k}^{\prime}(0)}{\phi_{k}^{\prime \prime}(0)} .
$$

Agora, se $d_{k}=-\nabla q\left(x_{k}\right)$, por (1.18) temos

$$
\alpha_{k}^{(0)}=\frac{\nabla q_{k}^{T} r_{k}}{r_{k}^{T} H r_{k}}=\frac{\left\|r_{k}\right\|_{2}^{2}}{r_{k}^{T} H r_{k}} .
$$

No caso $d_{k}=Z_{k} w_{j_{k}}$,

$$
\alpha_{k}^{(0)}=-\frac{\nabla q\left(x_{k}\right)^{T} Z_{k} w_{j_{k}}}{w_{j_{k}}^{T} Z_{k}^{T} H Z_{k} w_{j_{k}}}
$$

tendo por (1.11), que

$$
\alpha_{k}^{(0)}=-\frac{r_{k}^{T} w_{j_{k}}}{w_{j_{k}}^{T} H_{k} w_{j_{k}}} .
$$

Agora, como $w_{j_{k}}$ é o minimizador da função $q_{k}$, temos que $H_{k} w_{j_{k}}=-r_{k}$, concluíndo assim que $\alpha_{k}^{(0)}=1$.

Propriedade 2.1. Se $z \in \Omega$ satisfaz as condições $K K T$ de (2.8) então z também satisfaz as condições KKT de

$$
\begin{array}{ll}
\text { Minimizar } & f(x) \\
\text { sujeita a } & x \in \Omega(z, \delta),
\end{array}
$$

para todo $\delta \geq 0$. Reciprocamente, se z satisfaz as condições KKT de (2.9) para algum $\delta \geq 0$, então $z$ satisfaz as condições KKT de (2.8).

Prova: Se $z$ satisfaz as condições $\operatorname{KKT}$ de (2.8) então existem $[\mu]_{i} \geq 0, i=$ 
$1, \ldots, p$ tal que $[\mu]_{i} C_{i}(z)=0$ e

$$
\nabla f(z)+\sum_{i=1}^{p}[\mu]_{i} \nabla C_{i}(z)=0
$$

o que pode ser reformulado como

$$
\nabla f(z)+\sum_{i \in I(z, \delta)}[\mu]_{i} \nabla C_{i}(z)+\sum_{i \notin I(z, \delta)}[\mu]_{i} \nabla C_{i}(z)=0
$$

para todo $\delta \geq 0$. Se $i \notin I(z, \delta)$ então $C_{i}(z)<-[\delta]_{i} \leq 0$, pelo que $[\mu]_{i}=0$ para todo $i \notin I(z, \delta)$, provando assim que $\nabla f(z)+\sum_{i \in I(z, \delta)}[\mu]_{i} \nabla C_{i}(z)=0$, ou seja, que $z$ satisfaz as condições KKT de (2.9).

A prova da recíproca é análoga. 


\section{Referências Bibliográficas}

Andretta, M. Tópicos em otimização com restrições lineares. Tese de Doutoramento, IME, Universidade de São Paulo, São Paulo, SP, Brasil, 2008.

Andretta, M.; Birgin, E. G.; Martínez, J. M. Practical active-set Euclidian trust-region method with spectral projected gradients for boundconstrained minimization. Optimization, v. 54, p. 305-325, 2005.

Andretta, M.; Birgin, E. G.; Martínez, J. M. Partial spectral projected gradient method with active-set strategy for linearly constrained optimization. Numerical Algorithms, v. 53, p. 23-52, 2010.

Bartlett, R. A.; Biegler, L. T. QPSchur: a dual, active-set, Schurcomplement method for large-scale and structured convex quadratic programming. Optim. Eng., v. 7 (1), p. 5-32, 2006.

Barzilai, J.; Borwein, J. Two-point step size gradient methods. IMA Journal of Numerical Analysis, v. 8, p. 141-148, 1988.

Bertsekas, D. P. Nonlinear programming. Athena Scientific, Belmont, MA, 1999.

Bielschowski, R. H.; Friedlander, A.; Gomes, F. A. M.; MartíNEZ, J. M.; RAYDAN, M. An adaptive algorithm for bound constrained quadratic minimization. Investigación Operativa, v. 7, p. 67-102, 1997.

Birgin, E. G.; Martínez, J.; Raydan, M. Nonmonotone spectral projected gradient methods on convex sets. SIAM Journal on Optimization, v. 10, p. 1196-1211, 2000. 
Birgin, E. G.; Martínez, J.; Raydan, M. Algorithm 813: SPG - software for convex-constrained optimization. ACM Transactions on Mathematical Software, v. 27, p. 340-349, 2001.

Birgin, E. G.; Martínez, J. M.; Raydan, M. Inexact Spectral Projected Gradient methods on convex sets. IMA Journal on Numerical Analysis, v. 23, p. 539-559, 2003.

Boland, N. L. A dual-active-set algorithm for positive semi-definite quadratic programming. Mathematical Programming, v. 78 (1, ser. A), p. 1-27, 1997.

Bongartz, I.; Conn, A. R.; Gould, I. M.; Toint, P. L. CUTE constrained and unconstrained testing environment. ACM Transactions on Mathematical Software (TOMS)., v. 21, p. 123-160, 1995.

Calamai, P. H.; Moré, J. J. Projected gradient methods for linearly constrained problems. Math. Programming, v. 39, p. 93-116, 1987.

Coleman, T.; Hulbert., L. A direct active-set algorithm for large sparse quadratic programs with simple bounds. Mathematical Programming., v. 45, 1-3, p. 373-406, 1989.

Dai, Y.-H.; Fletcher, R. Projected Barzilai-Borwein methods for largescale box-constrained quadratic programming. Numerische Mathematik, v. 100 , p. 21-47, 2005.

DostÁL, Z. Box constrained quadratic programming with proportioning and projections. SIAM J. Optim, v. 7(3), p. 871-887, 1997.

DostáL, Z. A proportioning based algorithm with rate of convergence for bound constrained quadratic programming. Numerical Algorithms, v. 34, p. 293-302, 2003.

Dostál, Z.; SchöBerL, J. Minimizing quadratic functions over nonnegative cone with the rate of convergence and finite termination. Comput. Optim. Appl., v. 30, p. 23-44, 2005.

DufF, I. S. MA57 a code for the solution of sparse symmetric definite and indefinite systems. ACM Transactions on Mathematical Software (TOMS)., v. 30, p. 118-144, 2004. 
FISCHER, A. A special Newton-type optimization method. Optimization, v. 24, p. $269-284,1992$.

Friedlander, A.; Martínez, J. On the maximization of a concave quadratic function with box constraints. SIAM Journal on Optimization., v. 4, p. 177-192, 1994.

Friedlander, A.; Martínez, J.; Raydan, M. A new method for largescale box constrained convex quadratic minimization problems. Optimization Methods and Software., v. 5, p. 57-74, 1995.

Gaujoux, R. G. Resolução de sistema KKT por método de tipo Newton não diferenciável. Dissertação de Mestrado, IMECC, Universidade Estadual de Campinas, Campinas, SP, Brasil, 2005.

Gill, P.; Murray, W.; Saunders, M.; Wright, M. H. A Schurcomplement method for sparse quadratic programming. In Reliable Numerical Computation, Oxford University Press., p. 113-138, 1990.

Goldfarb, D.; IDnAni, A. A numerically stable dual method for solving strictly convex quadratic programs. Math. Programming, v. 27, p. 1-33, 1983.

Grippo, L.; LAmpariello, F.; Lucidi, S. A nonmonotone line search technique for Newton's method. SIAM J. Numer. Anal., v. 23, p. 707-716, 1986.

Moré, J. J.; Sorensen, D. C. Computing a trust region step. SIAM Journal on Scientific and Statistical Computing, v. 4, p. 553-572, 1983.

Moré, J. J.; Toraldo, G. On the solution of large quadratic programming problems with bound constraints. SIAM J. Optimization, v. 1, p. 93-113, 1991.

Nocedal, J.; Wright, S. J. Numerical optimization. Springer, New York, USA, 1999.

Powell, M. J. D. ZQPCVX, a FORTRAN subroutine for convex programming. Relatório Técnico, University of Cambridge, England, 1983.

Powell, M. J. D. On the quadratic-programming algorithm of Goldfarb and Idnani. Math. Programming Study, v. 25, p. 46-61, 1985. 
RAYDAn, M. On the Barzilai and Borwein choice of steplength for the gradient method. IMA Journal of Numerical Analysis, v. 13, p. 321-326, 1993.

Schittkowski, K. QL: a Fortran code for convex quadratic programming. User's Guide, version 2.1, 2004.

Zhou, B.; Gao, L.; DAI, Y. Monotone projected gradient methods for large-scale box constrained quadratic programming. Science in China series A: Mathematics, v. 49, p. 688-702, 2006. 